Supporting Information (SI)

\title{
Synthesis and Rhodium(II)-Mediated Cascade Cyclopropanation/Rearrangement/Isomerization of Diazo 2,3,5-Trisubstituted Furans: the Construction of Pentasubstituted Aromatic Compounds
}

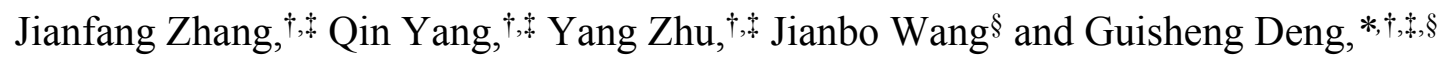

†Key Laboratory of Chemical Biology and Traditional Chinese Medicine Research (Ministry of Education of China), Hunan Normal University, Changsha 410081, China

Key Laboratory of the Assembly and Application of Organic Functional Molecules of Hunan Province, Hunan Normal University, Changsha 410081, China.

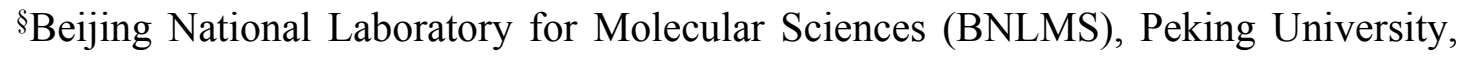
Beijing 100871, PR China

Table of Contents

1. ${ }^{1} \mathrm{H}$ NMR and ${ }^{13} \mathrm{C}$ NMR Spectra for New Products 2aa-gb, 3aa, 3fa, 3ha-ja, 3ab-cb, 3eb, 3gb, 4aa-ja, 4ab, 4cb, 4eb and 1aa-gb ..........................S2

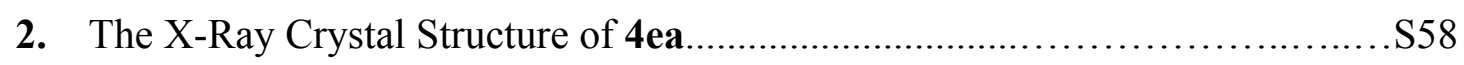


1. ${ }^{1} \mathrm{H}$ NMR and ${ }^{13} \mathrm{C}$ NMR Spectra for New Products

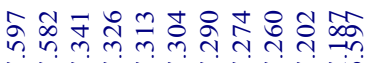

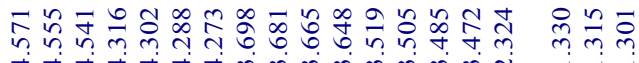

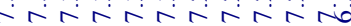

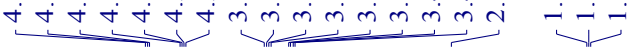

$\overbrace{\mathrm{O}}^{2} \mathrm{OEt}$

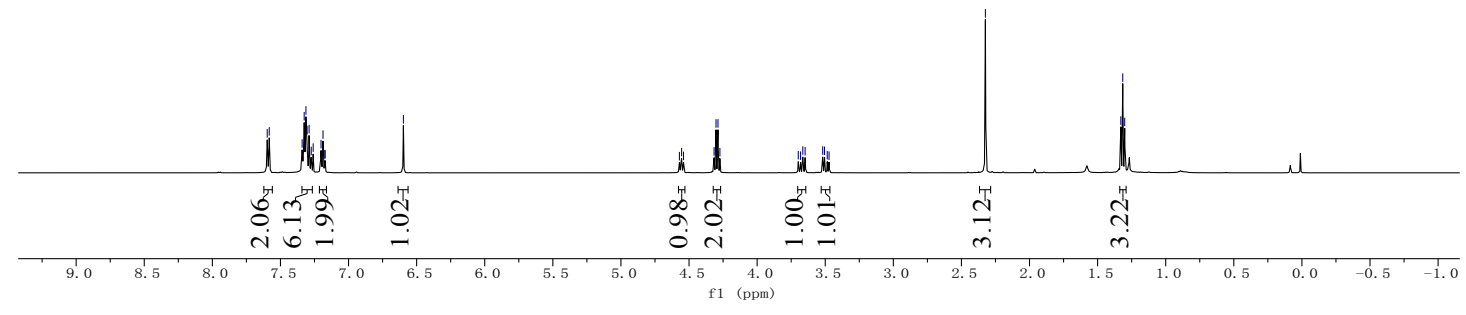

${ }^{1} \mathrm{H}$ NMR spectrum of $2 \mathbf{a a}\left(500 \mathrm{MHz}, \mathrm{CDCl}_{3}\right)$

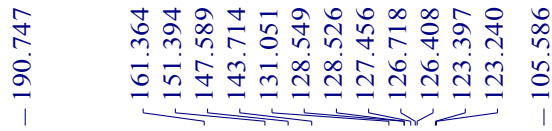

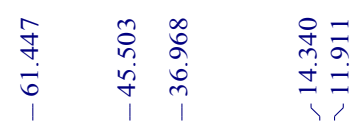<smiles>CCOC(=O)C(=N)C(=O)CC(c1ccccc1)c1cc(-c2ccccc2)oc1C</smiles>
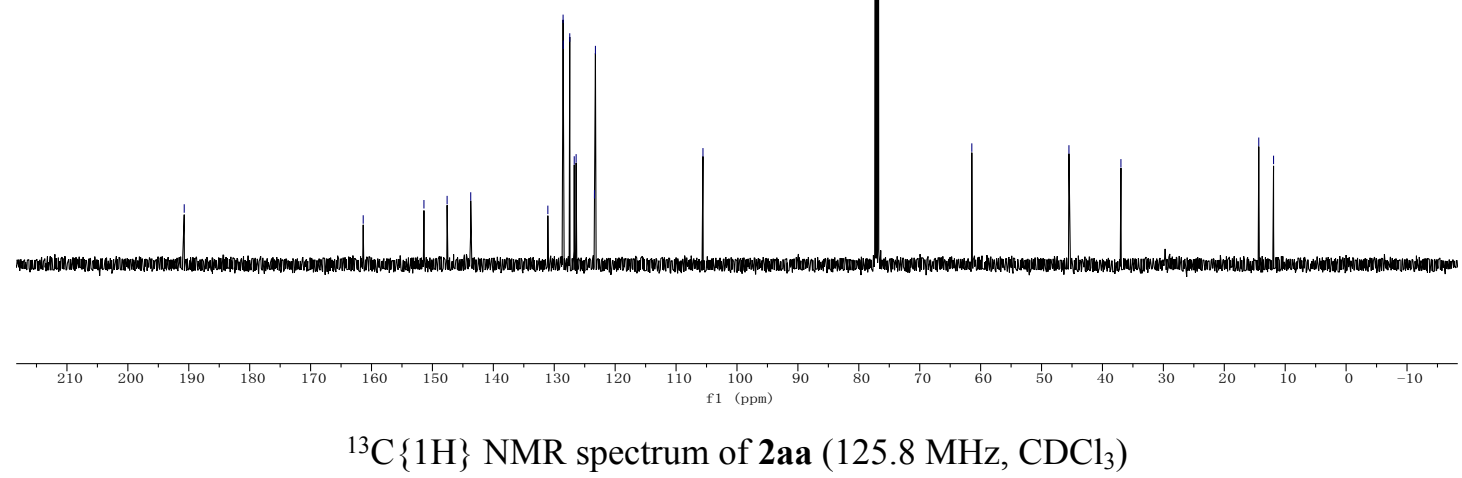

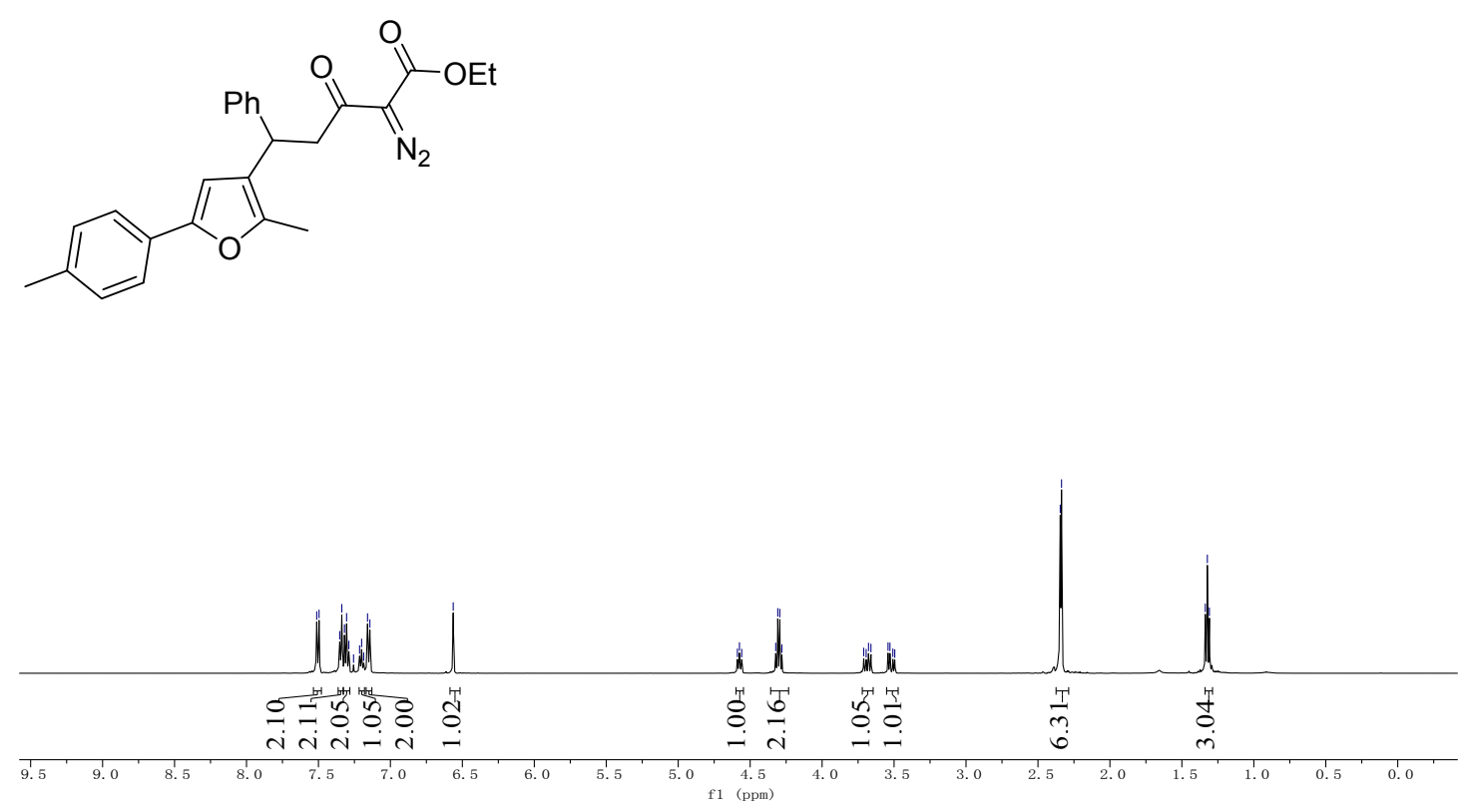

${ }^{1} \mathrm{H}$ NMR spectrum of $2 \mathbf{b a}\left(500 \mathrm{MHz}, \mathrm{CDCl}_{3}\right)$

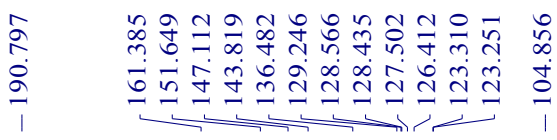<smiles>CCOC(=O)C(=N)C(=O)CC(c1ccccc1)c1cc(-c2ccc(C)cc2)oc1C</smiles>
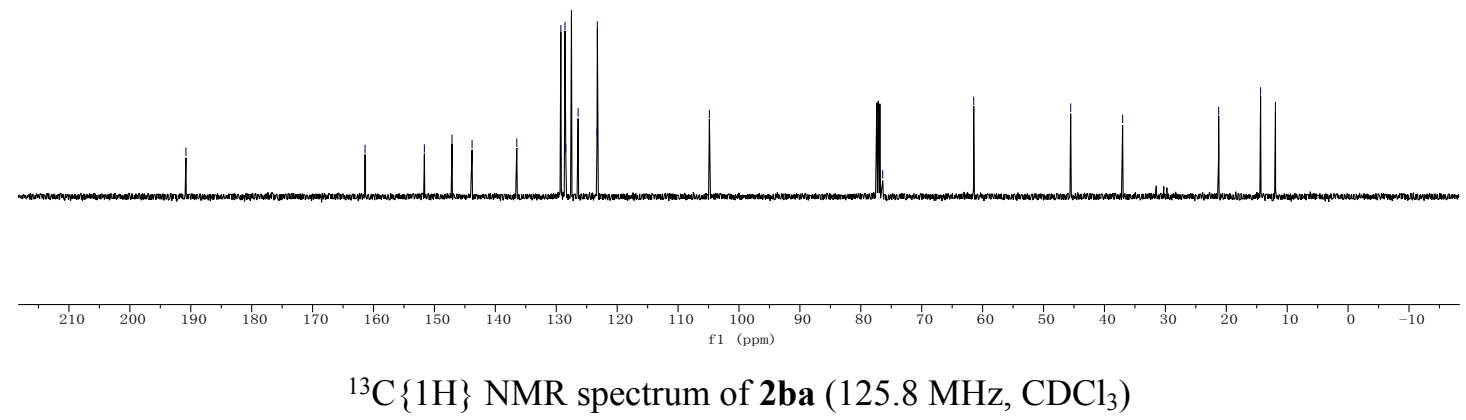
<smiles>CCOC(=O)C(=N)C(=O)CC(c1ccccc1)c1cc(-c2ccc(OC)cc2)oc1C</smiles>

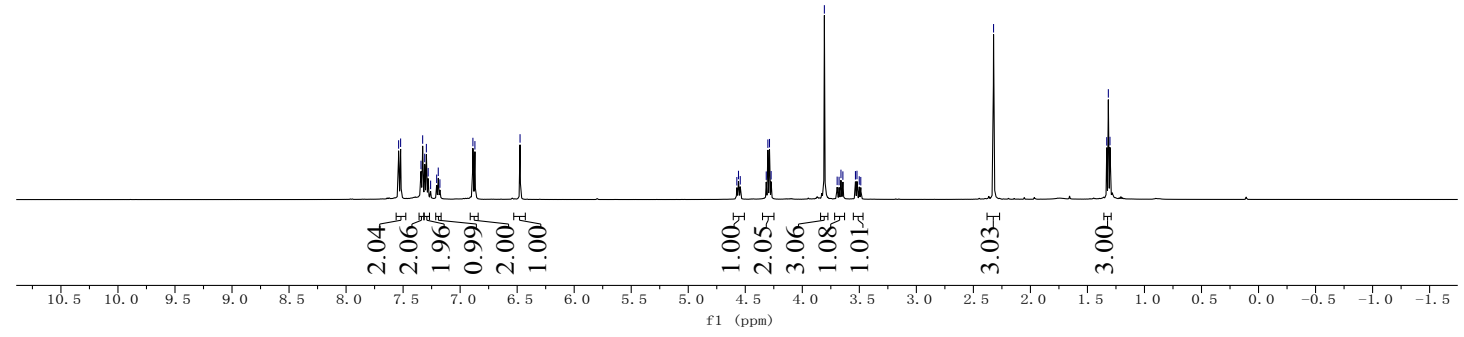

${ }^{1} \mathrm{H}$ NMR spectrum of $\mathbf{2 c a}\left(500 \mathrm{MHz}, \mathrm{CDCl}_{3}\right)$
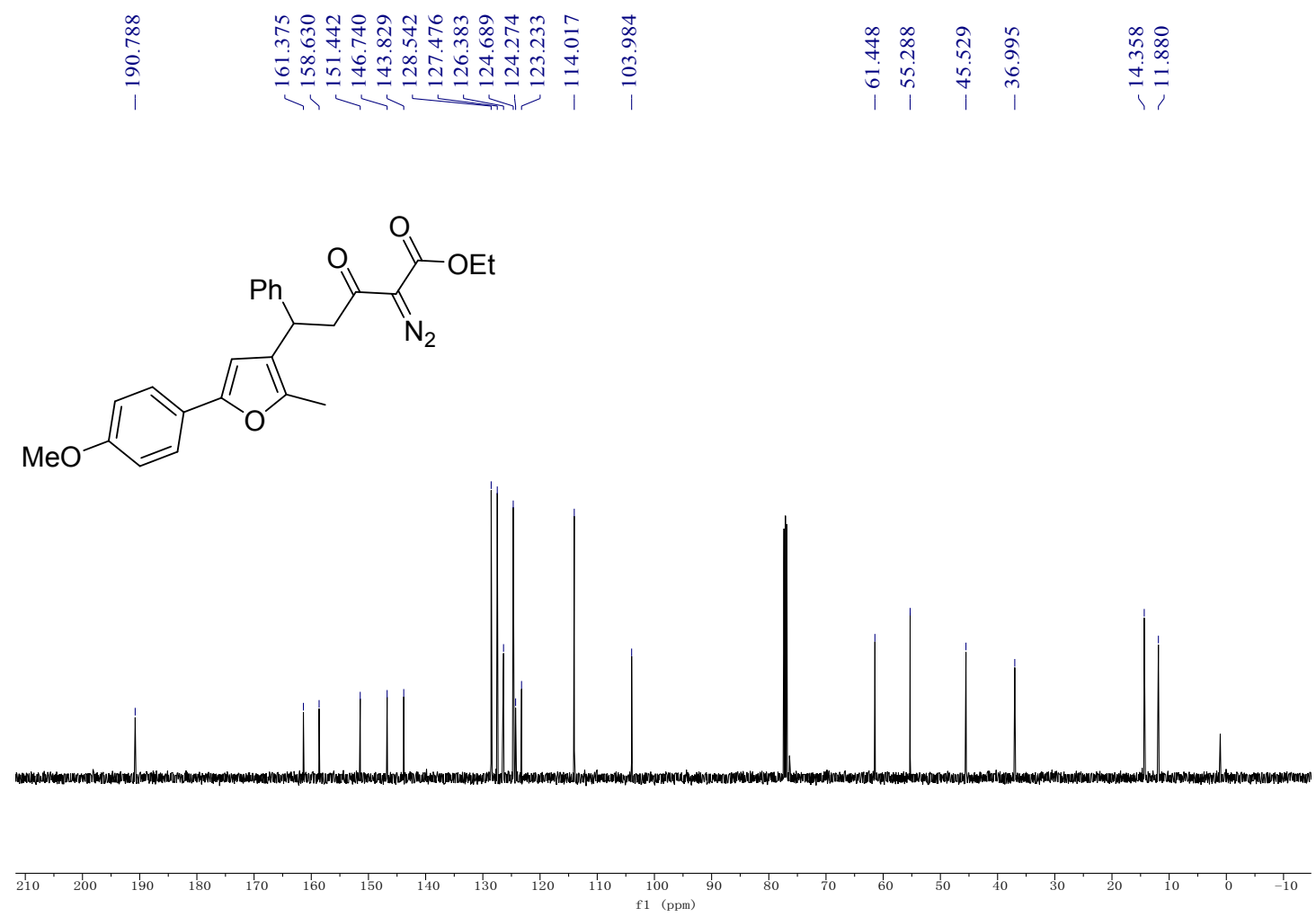

${ }^{13} \mathrm{C}\{1 \mathrm{H}\}$ NMR spectrum of $\mathbf{2} \mathbf{c a}\left(125.8 \mathrm{MHz}, \mathrm{CDCl}_{3}\right)$ 
<smiles>CCOC(=O)C(=N)C(=O)CC(c1ccccc1)c1cc(-c2ccc(Br)cc2)oc1C</smiles>

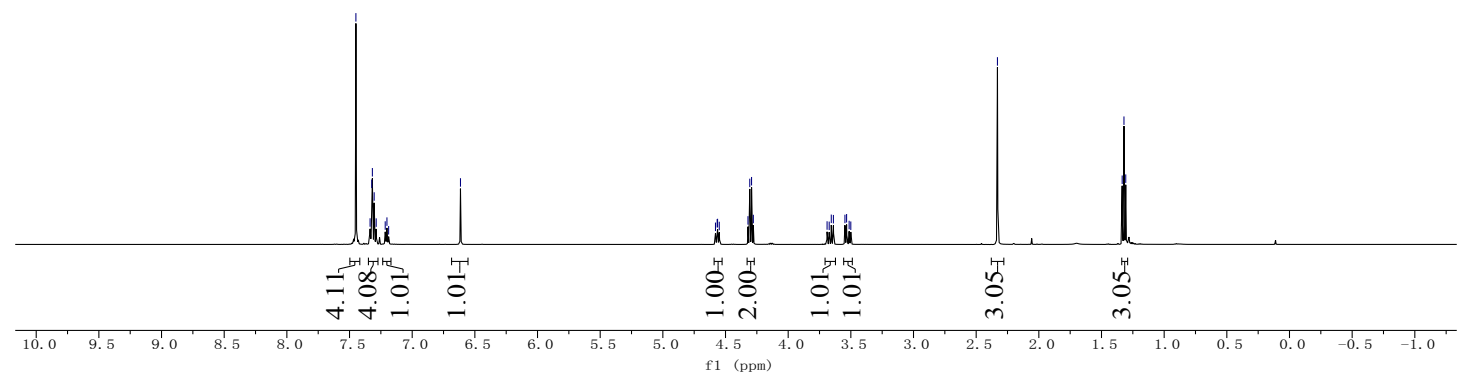

${ }^{1} \mathrm{H}$ NMR spectrum of $2 \mathbf{d a}\left(500 \mathrm{MHz}, \mathrm{CDCl}_{3}\right)$

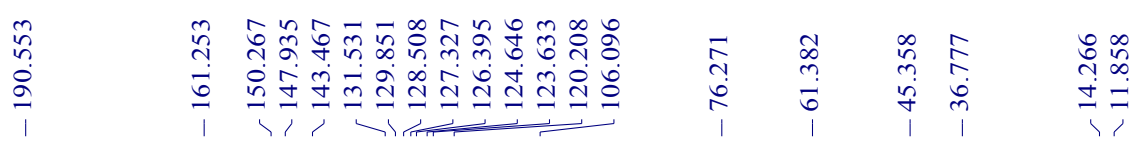<smiles>CCOC(=O)C(=N)C(=O)CC(c1ccccc1)c1cc(-c2ccc(Br)cc2)oc1C</smiles>
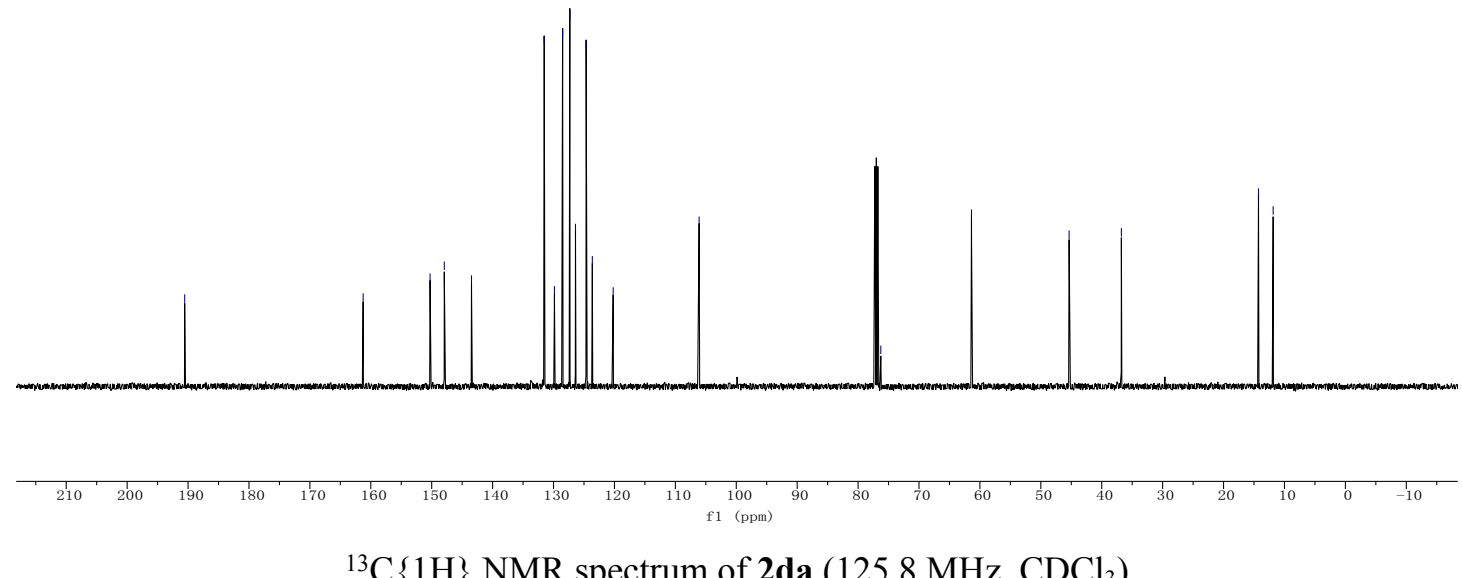

${ }^{13} \mathrm{C}\{1 \mathrm{H}\}$ NMR spectrum of $2 \mathbf{d a}\left(125.8 \mathrm{MHz}, \mathrm{CDCl}_{3}\right)$ 
<smiles>CCOC(=O)C(=N)C(=O)CC(c1ccccc1)c1cc(-c2ccc(Cl)cc2)oc1C</smiles>

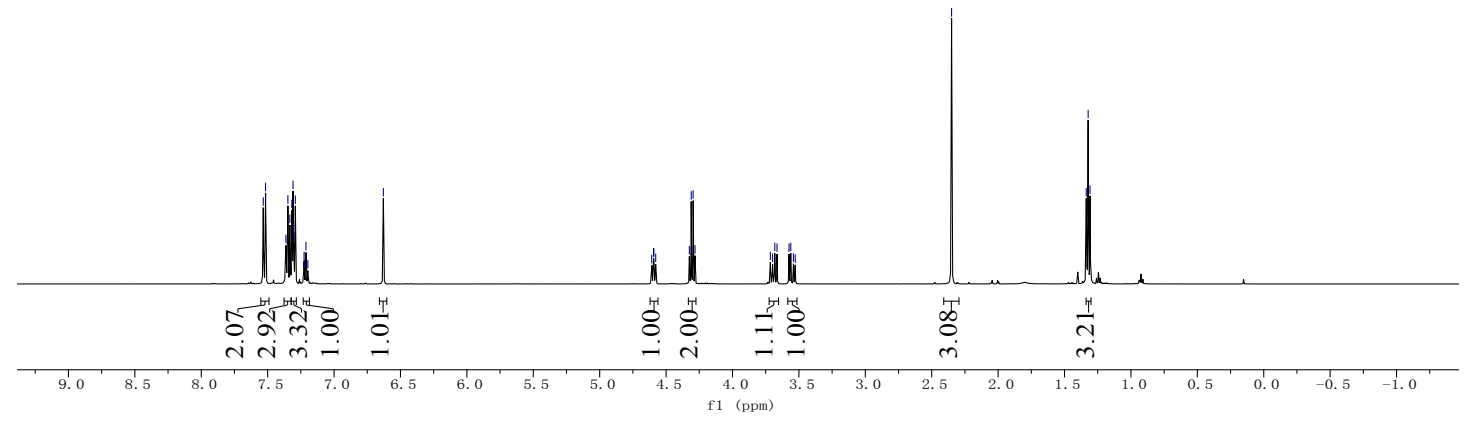

${ }^{1} \mathrm{H}$ NMR spectrum of 2 ea $\left(500 \mathrm{MHz}, \mathrm{CDCl}_{3}\right)$

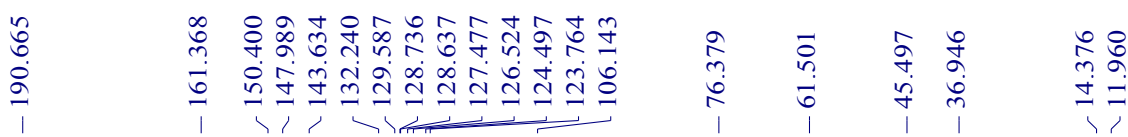<smiles>CCOC(=O)C(=N)C(=O)CC(c1ccccc1)c1cc(-c2ccc(Cl)cc2)oc1C</smiles>
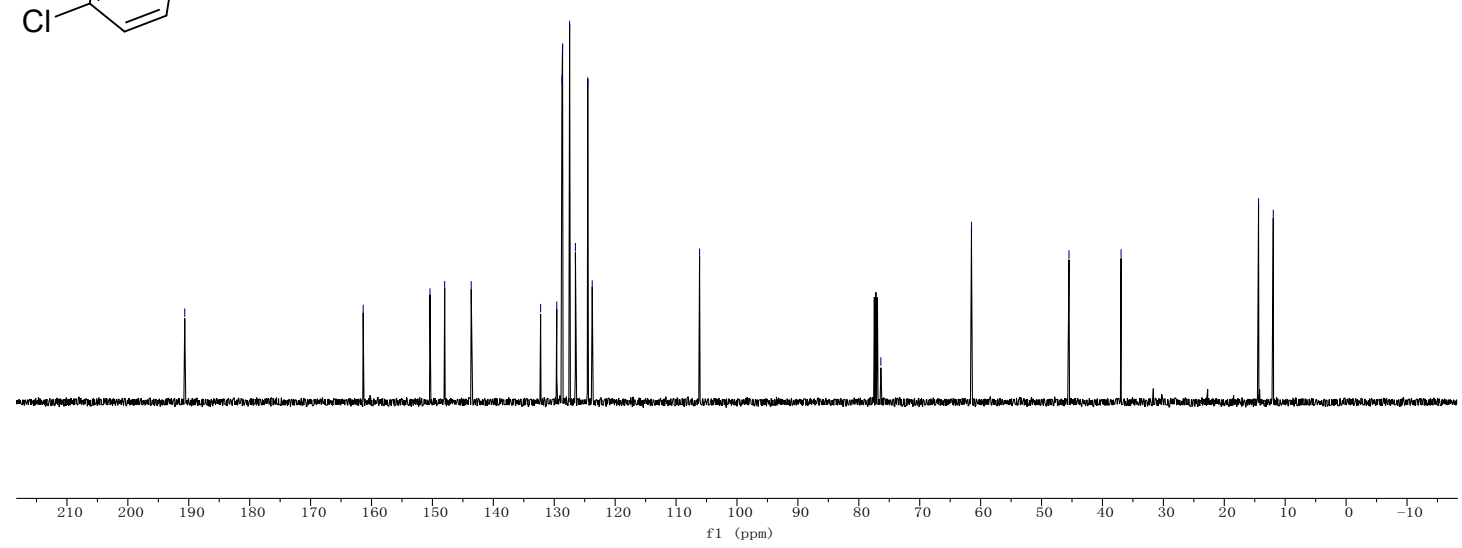

${ }^{13} \mathrm{C}\{1 \mathrm{H}\} \mathrm{NMR}$ spectrum of $2 \mathbf{e a}\left(125.8 \mathrm{MHz}, \mathrm{CDCl}_{3}\right)$ 


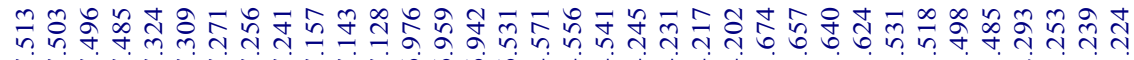

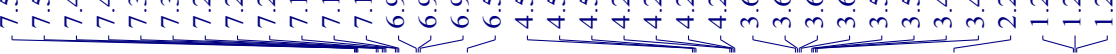<smiles>CCOC(=O)C(=N)C(=O)CC(c1ccccc1)c1cc(-c2ccc(F)cc2)oc1C</smiles>

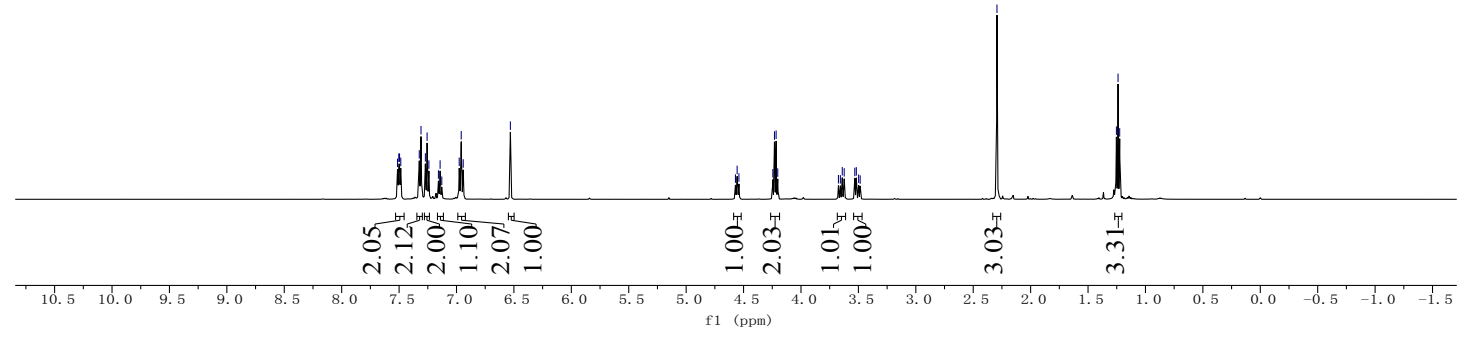

${ }^{1} \mathrm{H}$ NMR spectrum of $\mathbf{2} \mathbf{f a}\left(500 \mathrm{MHz}, \mathrm{CDCl}_{3}\right)$

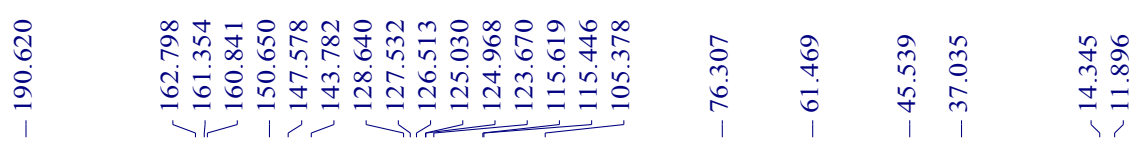<smiles>CCOC(=O)C(=N)C(=O)CC(c1ccccc1)c1cc(-c2ccc(F)cc2)oc1C</smiles>
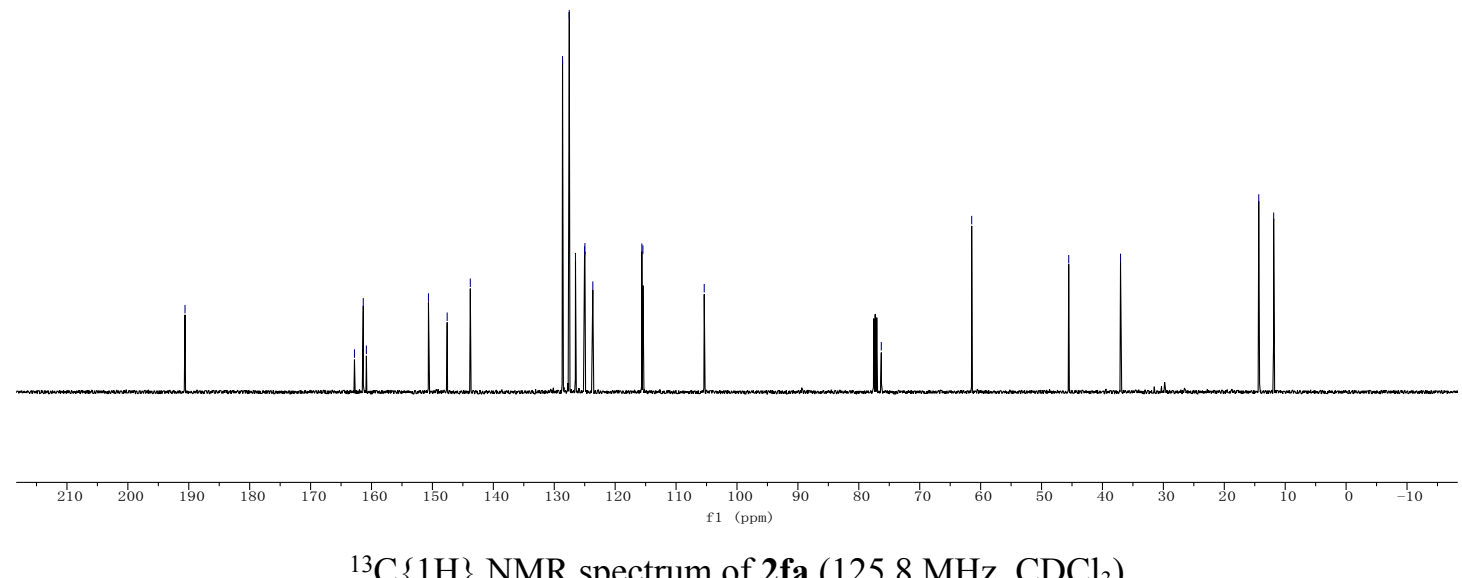

${ }^{13} \mathrm{C}\{1 \mathrm{H}\}$ NMR spectrum of $\mathbf{2} \mathbf{f a}\left(125.8 \mathrm{MHz}, \mathrm{CDCl}_{3}\right)$ 

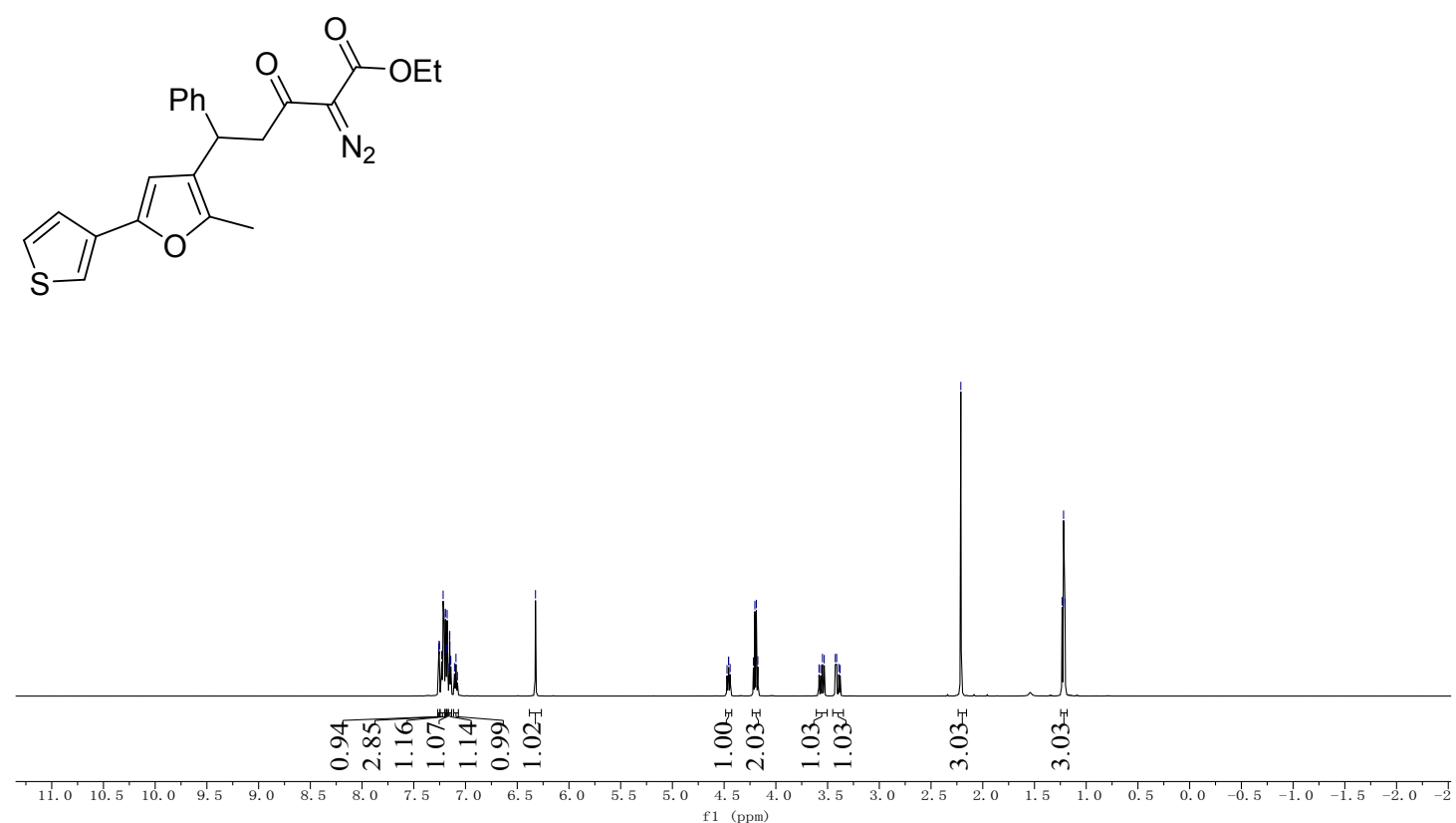

${ }^{1} \mathrm{H}$ NMR spectrum of 2 ga $\left(500 \mathrm{MHz}, \mathrm{CDCl}_{3}\right)$

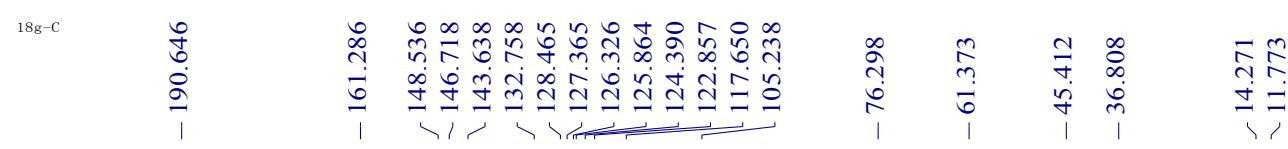<smiles>CCOC(=O)C(=N)C(=O)CC(c1ccccc1)c1cc(-c2ccsc2)oc1C</smiles>
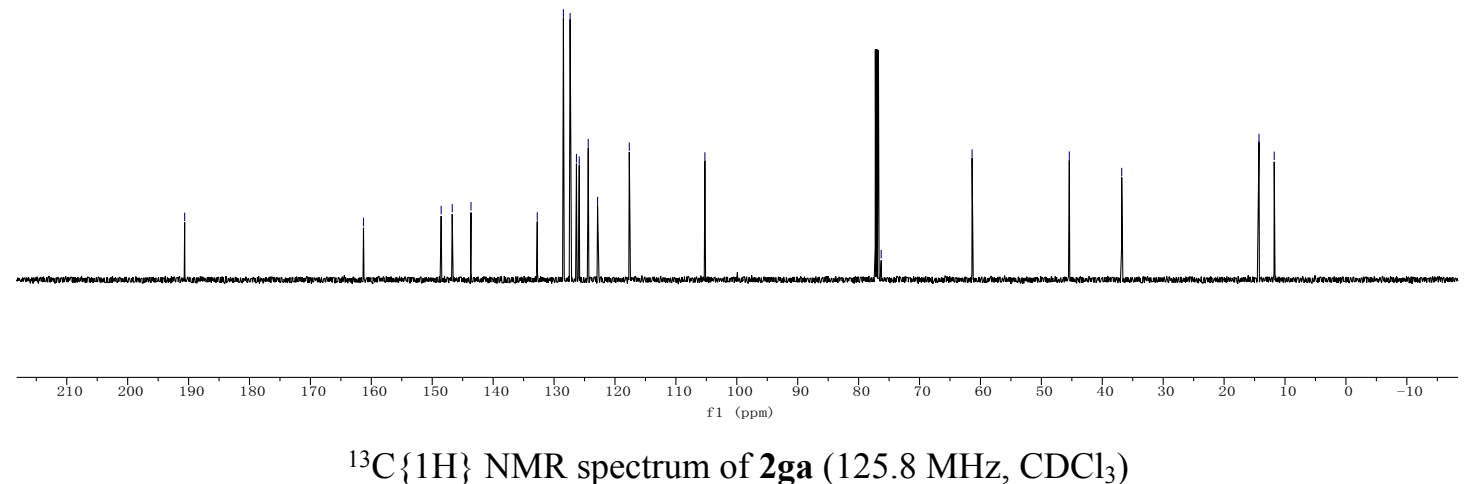

${ }^{13} \mathrm{C}\{1 \mathrm{H}\}$ NMR spectrum of 2 ga $\left(125.8 \mathrm{MHz}, \mathrm{CDCl}_{3}\right)$ 

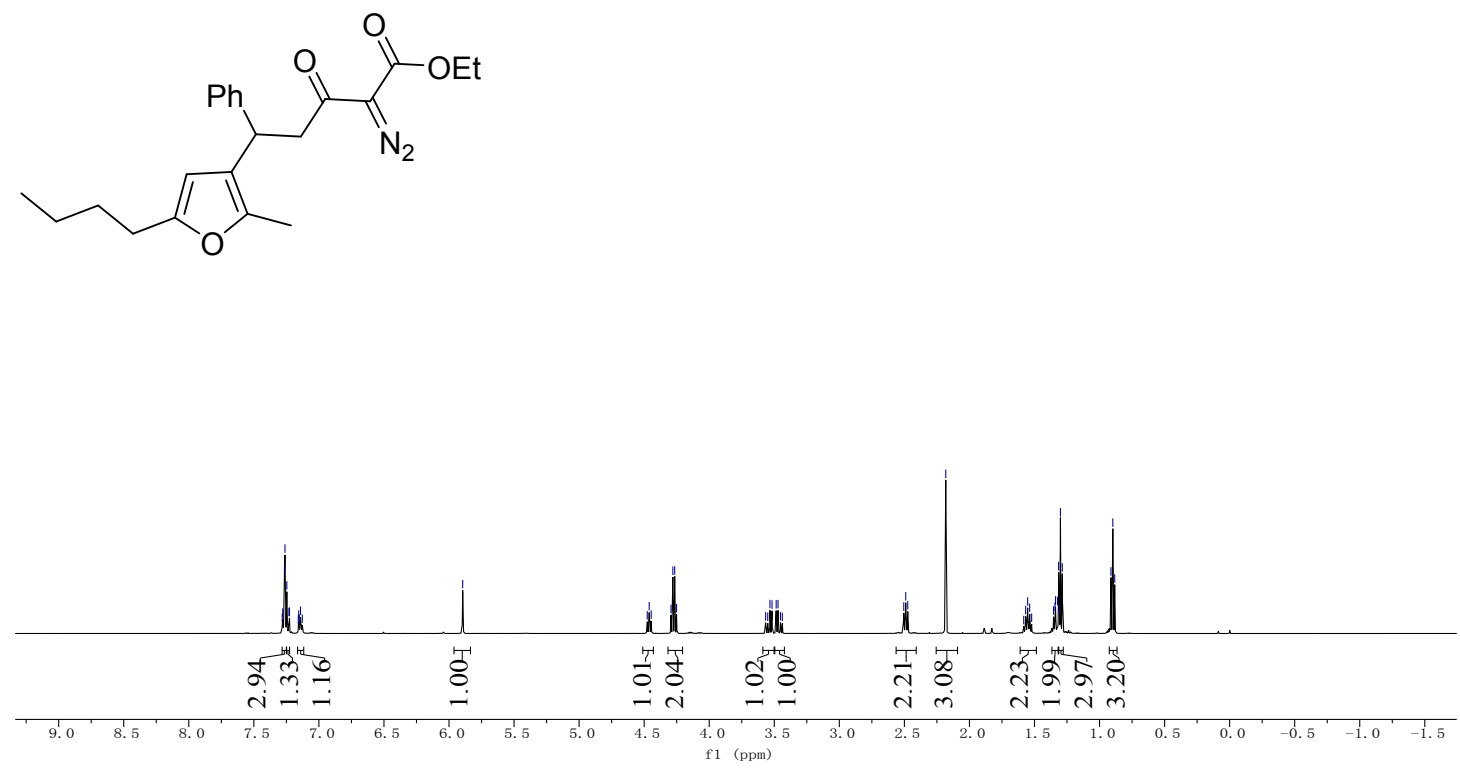

${ }^{1} \mathrm{H}$ NMR spectrum of $\mathbf{2 h a}\left(500 \mathrm{MHz}, \mathrm{CDCl}_{3}\right)$

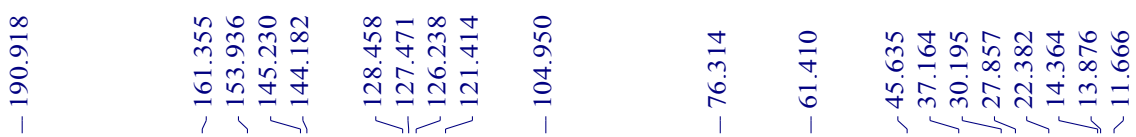<smiles>CCCCc1cc(C(CC(=O)C(=N)C(=O)OCC)c2ccccc2)c(C)o1</smiles>
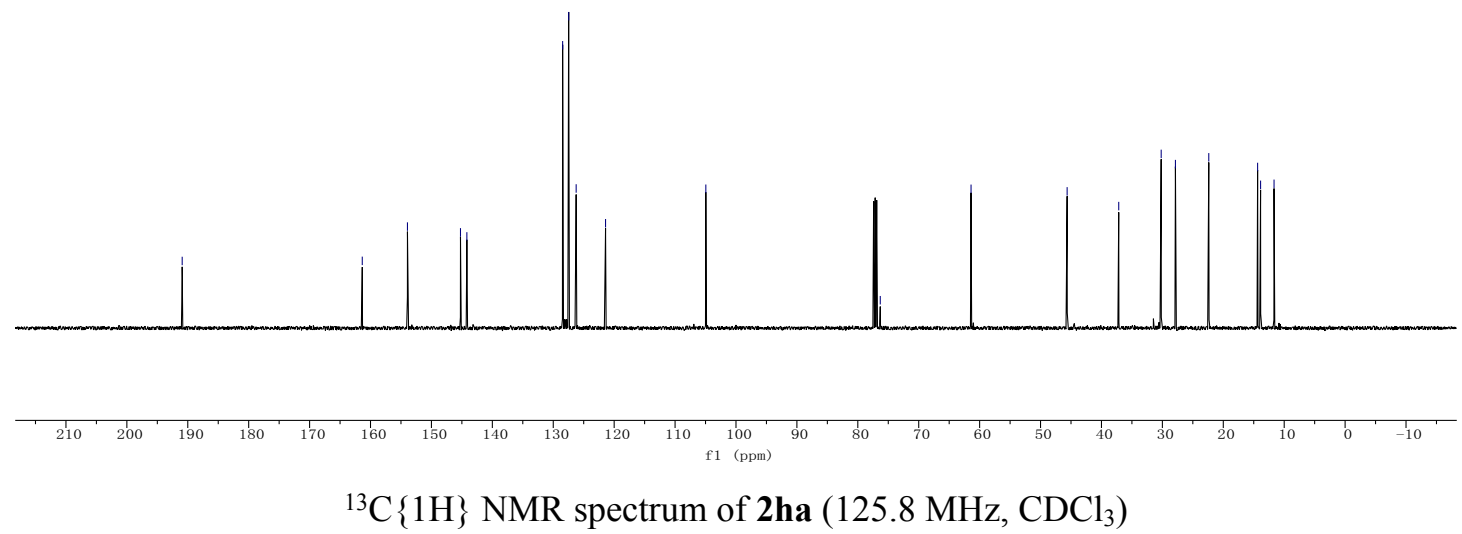


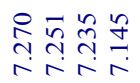
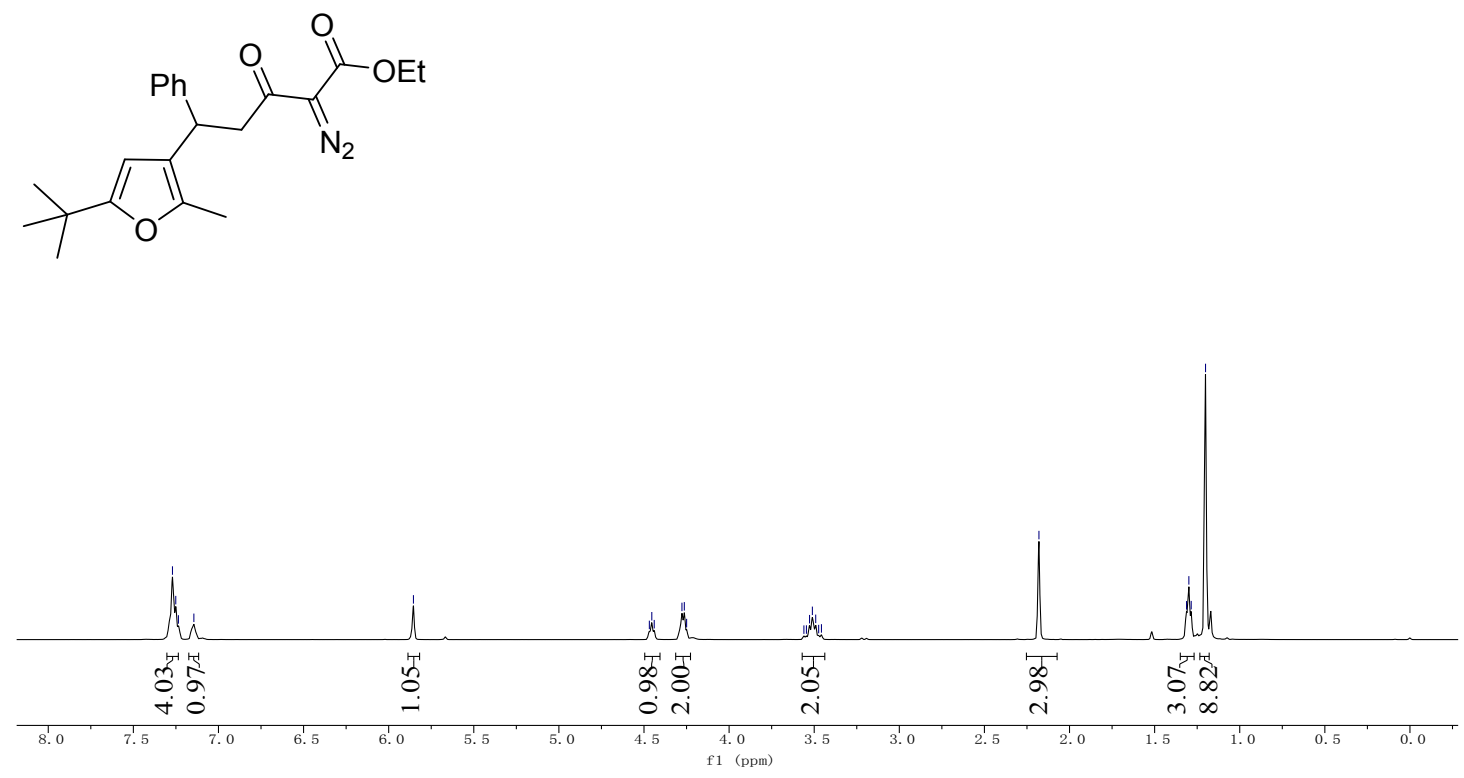

${ }^{1} \mathrm{H}$ NMR spectrum of 2 ia $\left(500 \mathrm{MHz}, \mathrm{CDCl}_{3}\right)$

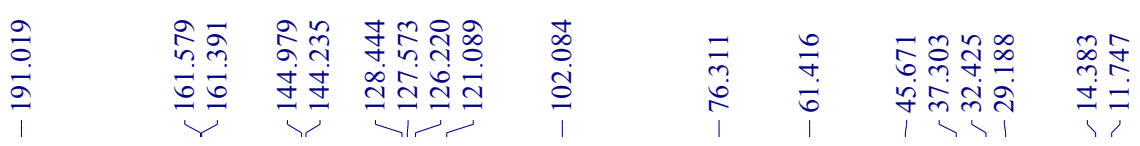<smiles>CCOC(=O)C(=N)C(=O)CC(c1ccccc1)c1cc(C(C)(C)C)oc1C</smiles>
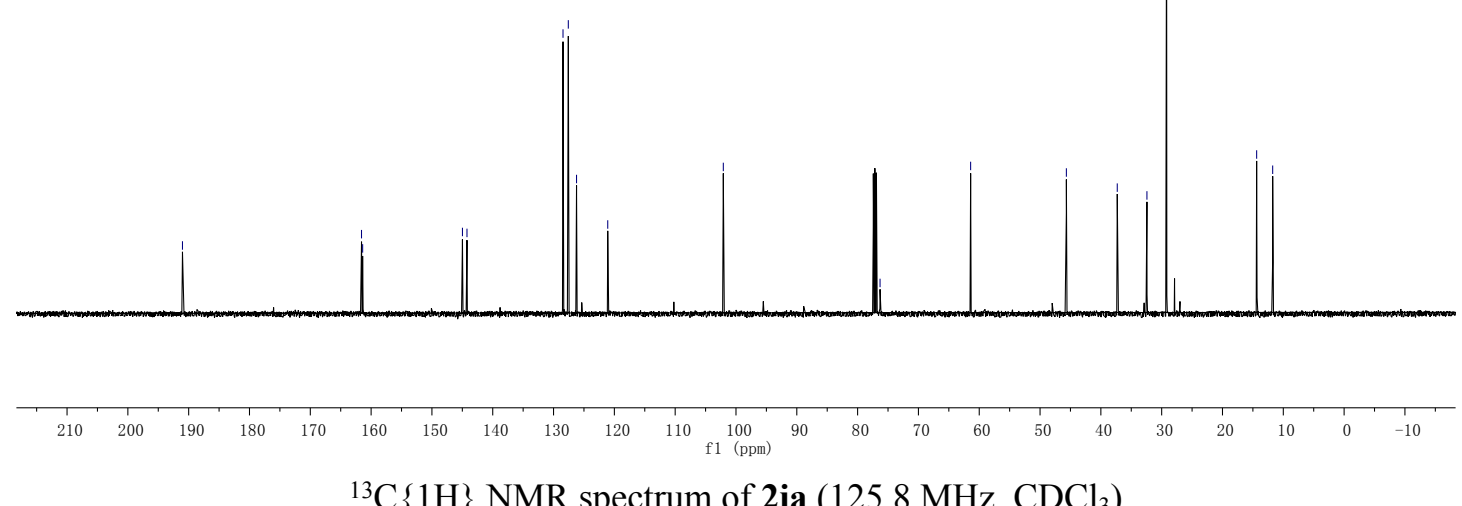

${ }^{13} \mathrm{C}\{1 \mathrm{H}\}$ NMR spectrum of 2 ia $\left(125.8 \mathrm{MHz} \mathrm{CDCl}_{3}\right)$ 
<smiles>CCOC(=O)C(=N)C(=O)CC(c1ccccc1)c1ccoc1C</smiles>

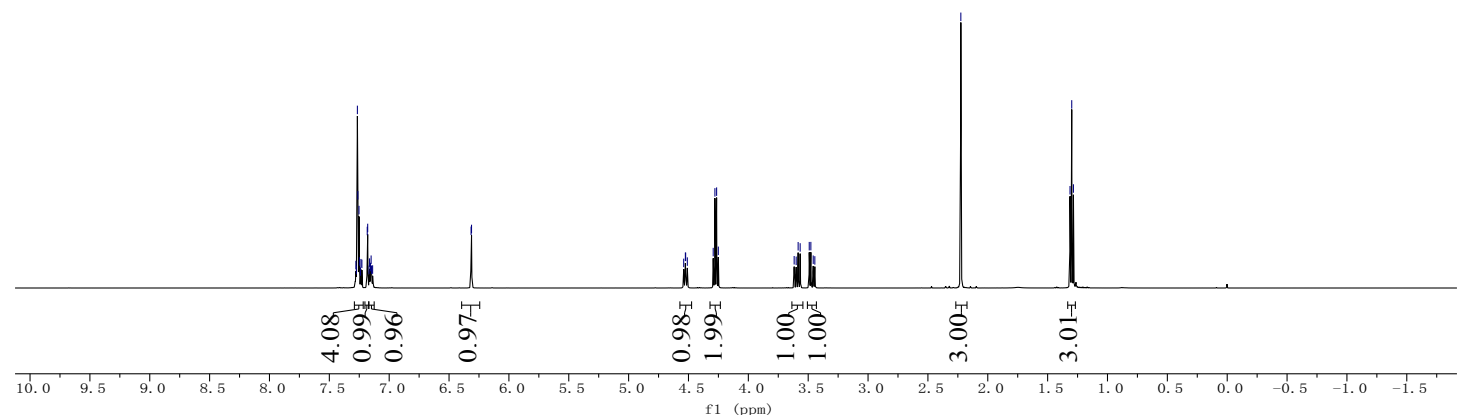

${ }^{1} \mathrm{H}$ NMR spectrum of $\mathbf{2} \mathbf{j a}\left(500 \mathrm{MHz}, \mathrm{CDCl}_{3}\right)$

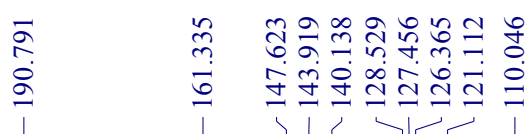<smiles>CCOC(=O)C(=N)C(=O)CC(c1ccccc1)c1ccoc1C</smiles>
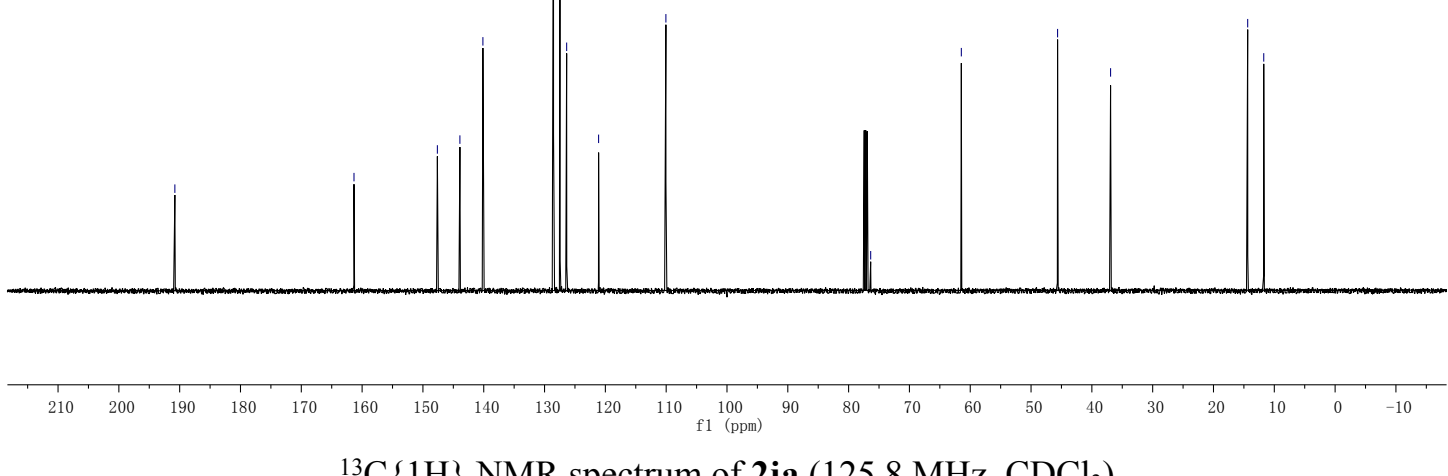

${ }^{13} \mathrm{C}\{1 \mathrm{H}\}$ NMR spectrum of $\mathbf{2} \mathbf{j a}\left(125.8 \mathrm{MHz}, \mathrm{CDCl}_{3}\right)$ 
<smiles>Cc1oc(-c2ccccc2)cc1C(CC(=O)C(=N)C(=O)c1ccccc1)c1ccccc1</smiles>

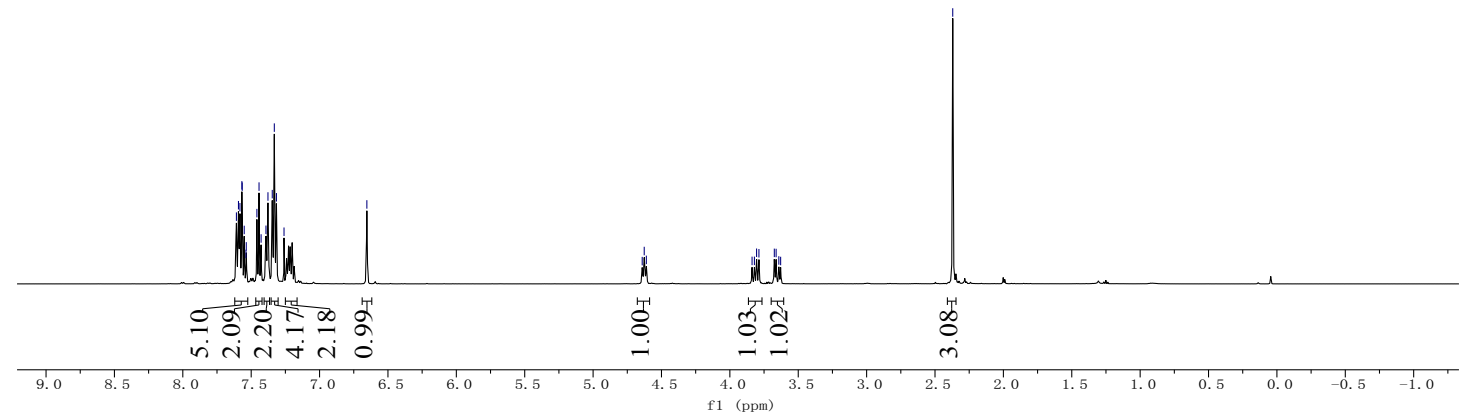

${ }^{1} \mathrm{H}$ NMR spectrum of $\mathbf{2} \mathbf{a b}\left(500 \mathrm{MHz}, \mathrm{CDCl}_{3}\right)$
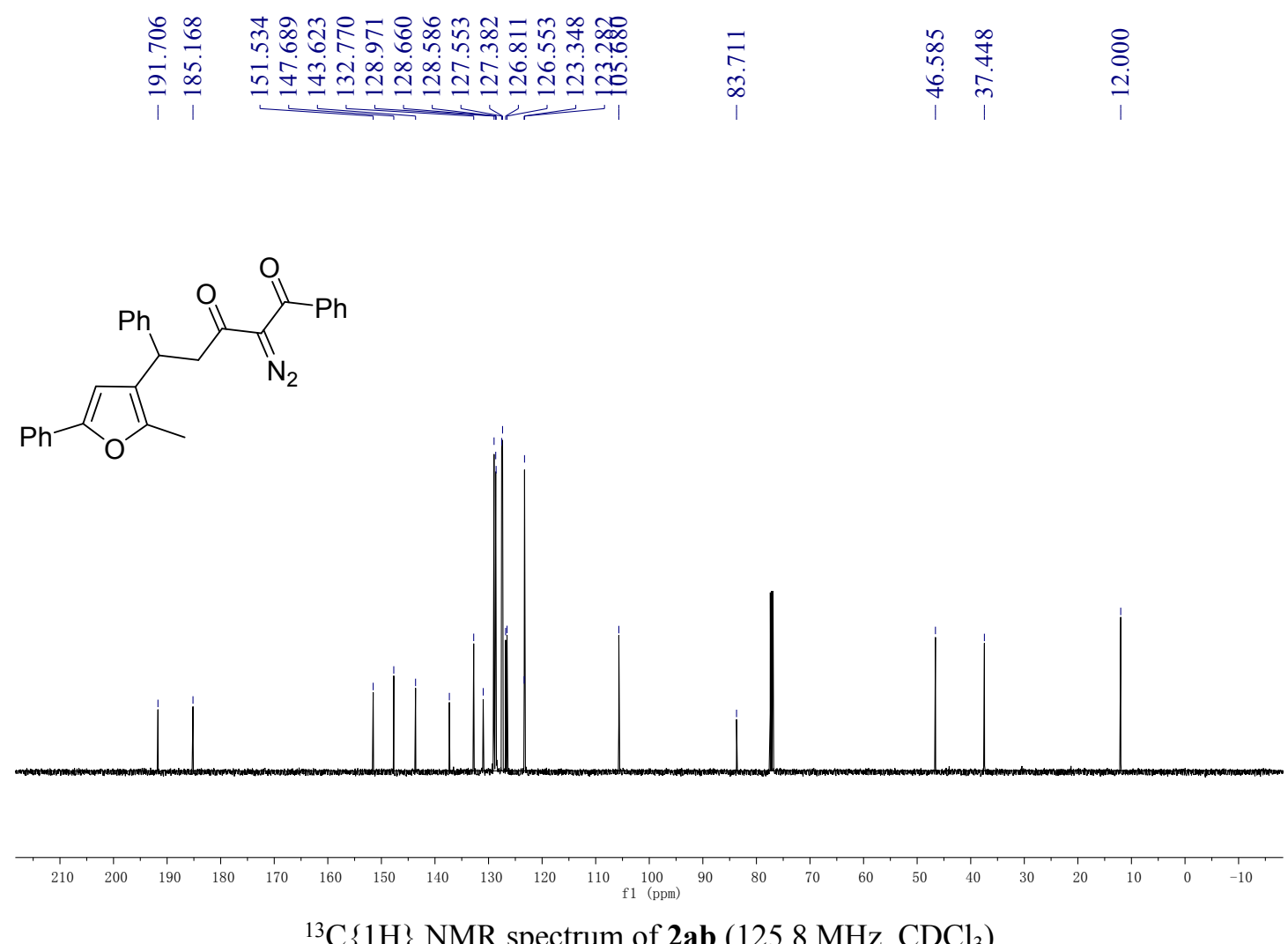

${ }^{13} \mathrm{C}\{1 \mathrm{H}\} \mathrm{NMR}$ spectrum of $\mathbf{2 a b}\left(125.8 \mathrm{MHz}, \mathrm{CDCl}_{3}\right)$ 

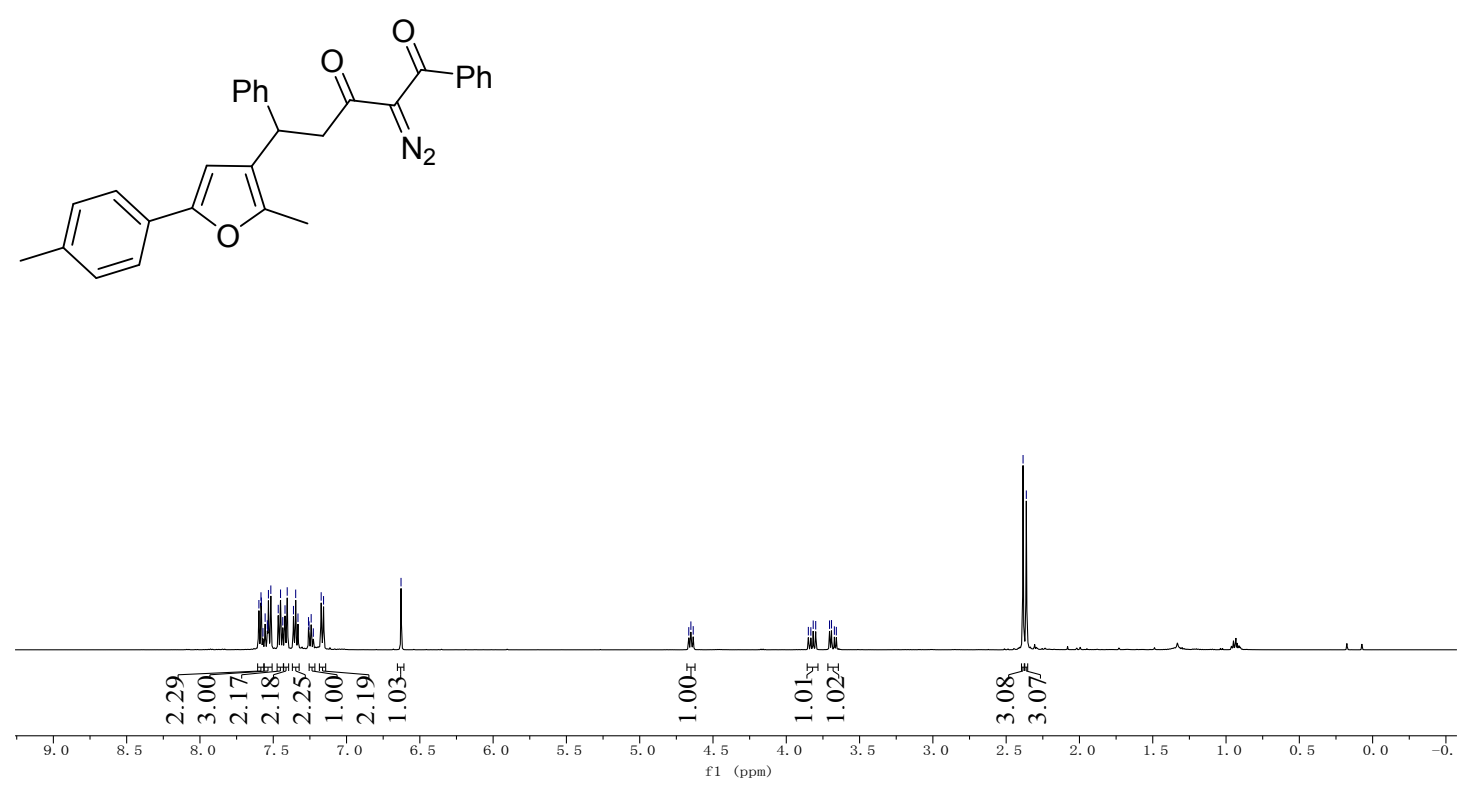

${ }^{1} \mathrm{H}$ NMR spectrum of $\mathbf{2 b b}\left(500 \mathrm{MHz}, \mathrm{CDCl}_{3}\right)$

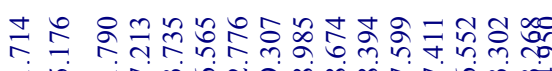

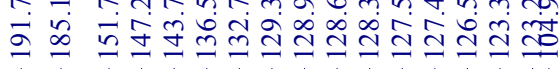
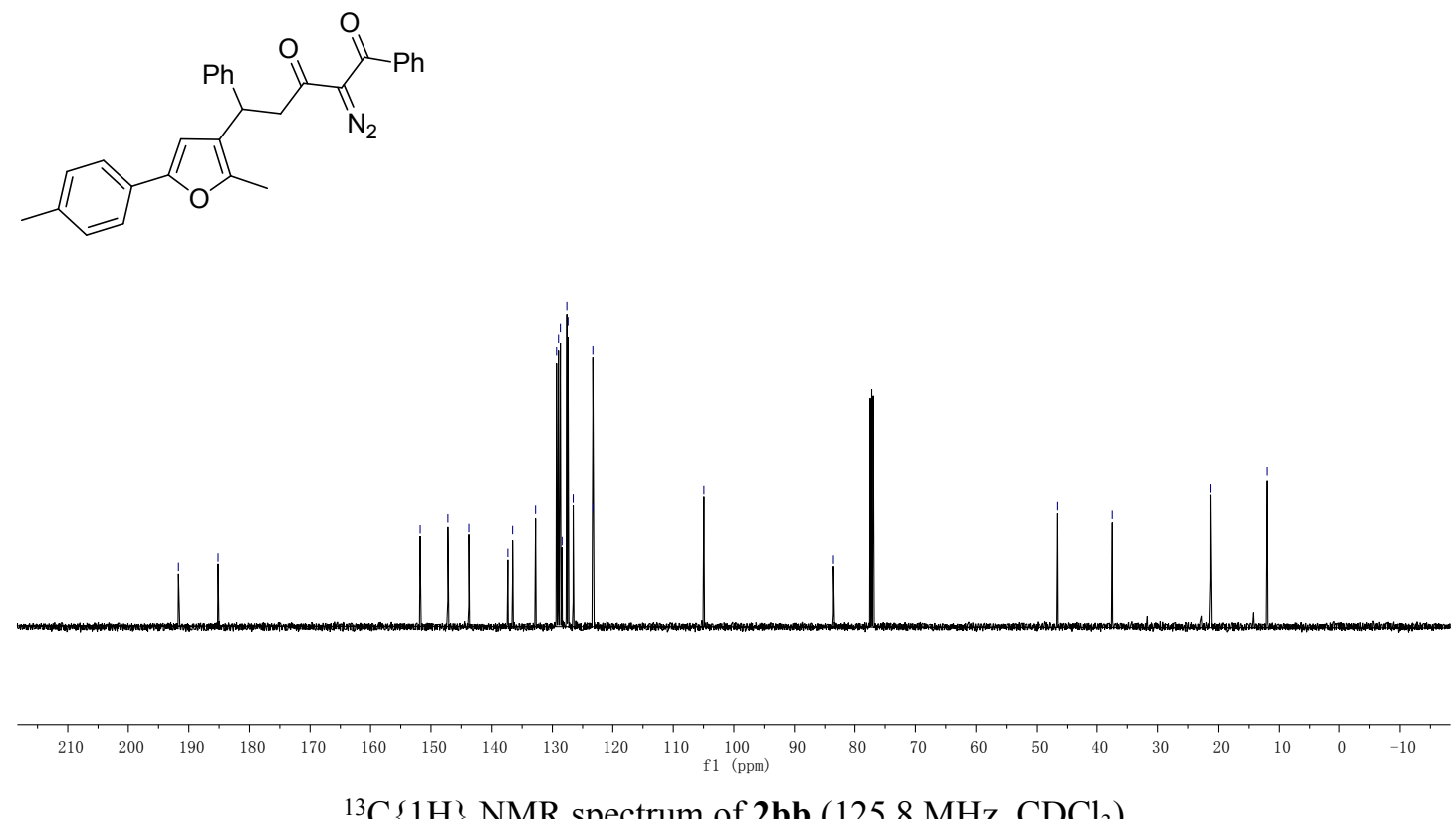

${ }^{13} \mathrm{C}\{1 \mathrm{H}\} \mathrm{NMR}$ spectrum of $\mathbf{2 b b}\left(125.8 \mathrm{MHz}, \mathrm{CDCl}_{3}\right)$ 

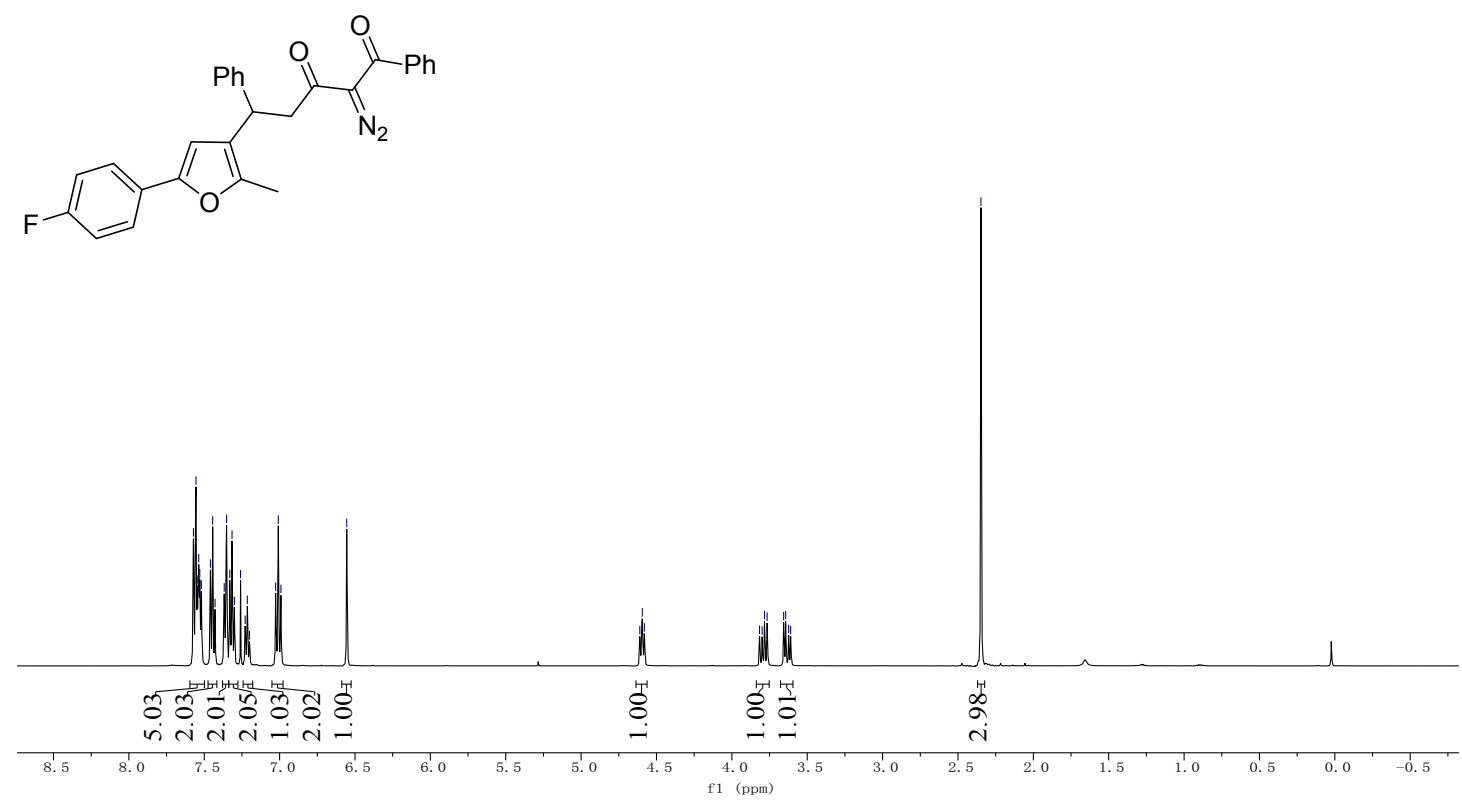

${ }^{1} \mathrm{H}$ NMR spectrum of $\mathbf{2} \mathbf{c b}\left(500 \mathrm{MHz}, \mathrm{CDCl}_{3}\right)$<smiles>Cc1oc(-c2ccc(F)cc2)cc1C(CC(=O)C(=O)c1ccccc1)c1ccccc1</smiles>
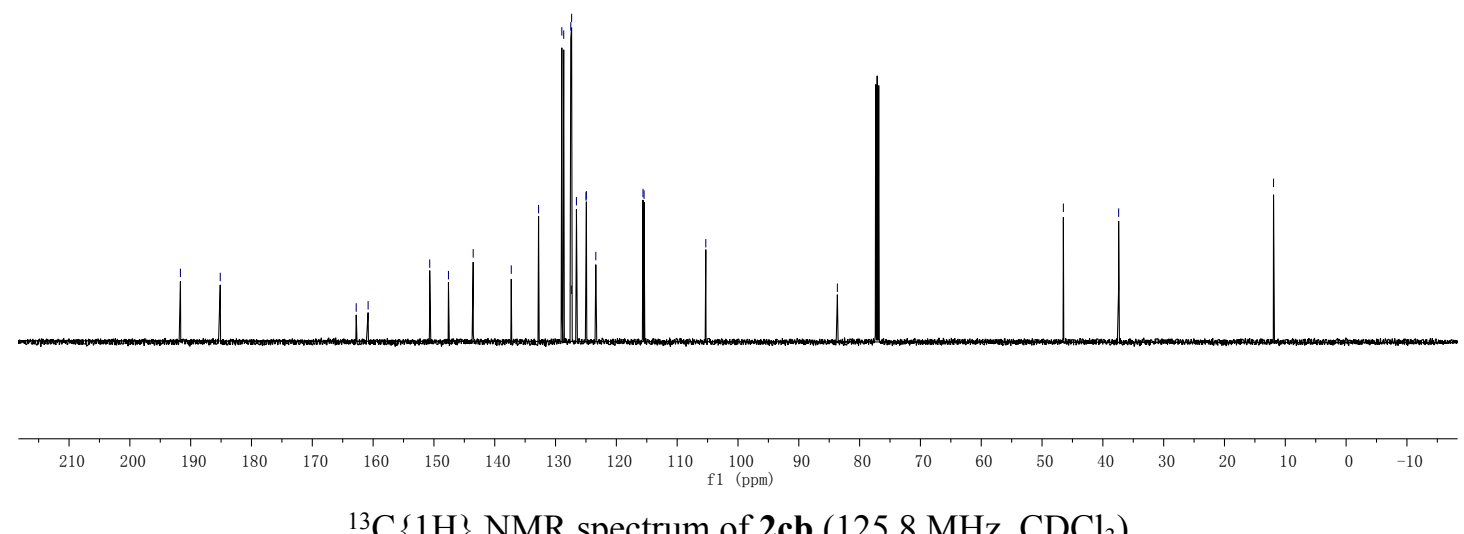

${ }^{13} \mathrm{C}\{1 \mathrm{H}\}$ NMR spectrum of $\mathbf{2} \mathbf{c b}\left(125.8 \mathrm{MHz}, \mathrm{CDCl}_{3}\right)$ 
<smiles>Cc1oc(-c2ccsc2)cc1C(CC(=O)C(=N)C(=O)c1ccccc1)c1ccccc1</smiles>

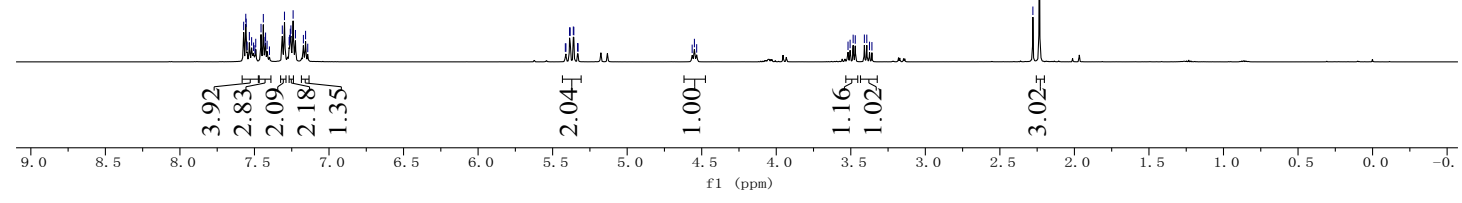

${ }^{1} \mathrm{H}$ NMR spectrum of $\mathbf{2} \mathbf{d b}\left(500 \mathrm{MHz}, \mathrm{CDCl}_{3}\right)$

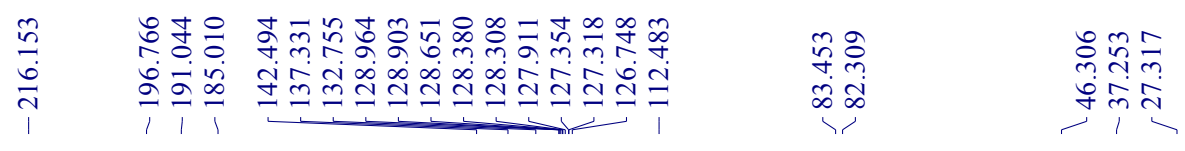<smiles>Cc1oc(-c2ccsc2)cc1C(CC(=O)C(=N)c1ccccc1)c1ccccc1</smiles>

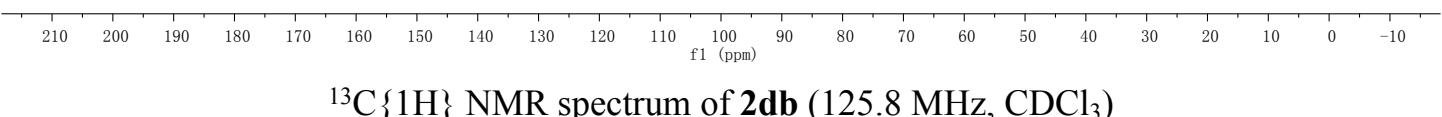

${ }^{13} \mathrm{C}\{1 \mathrm{H}\}$ NMR spectrum of $\mathbf{2 d b}\left(125.8 \mathrm{MHz}, \mathrm{CDCl}_{3}\right)$ 
<smiles>Cc1cc(C(CC(=O)C(=N)C(=O)c2ccccc2)c2ccccc2)c(C)o1</smiles>

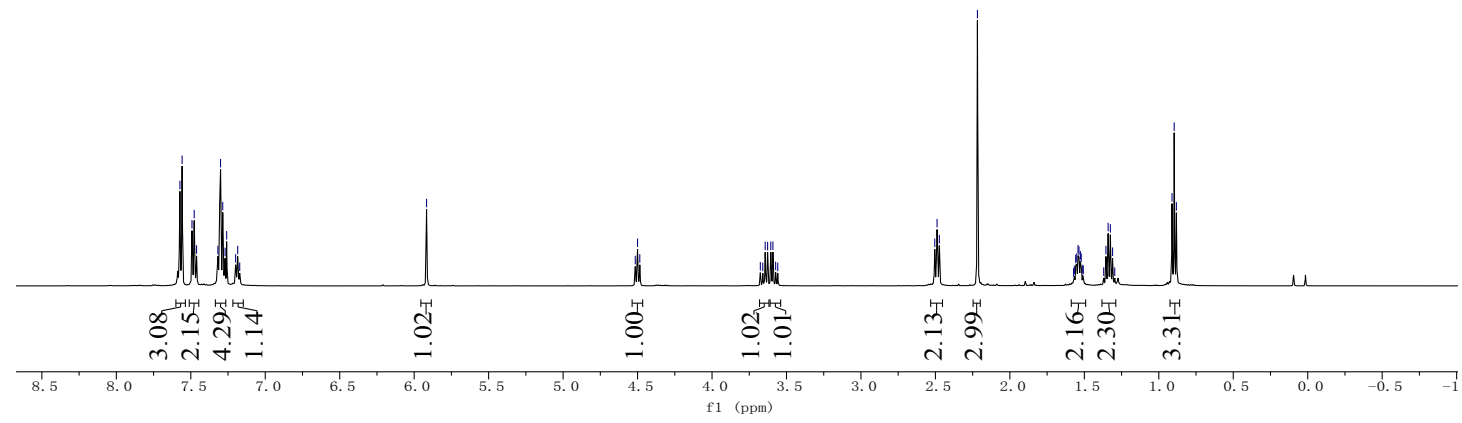

${ }^{1} \mathrm{H}$ NMR spectrum of $\mathbf{2 e b}\left(500 \mathrm{MHz}, \mathrm{CDCl}_{3}\right)$

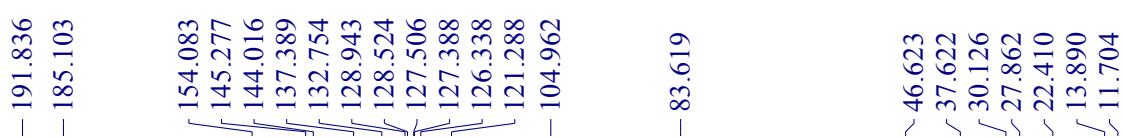<smiles>Cc1cc(C(CC(=O)C(=N)C(=O)c2ccccc2)c2ccccc2)c(C)o1</smiles>
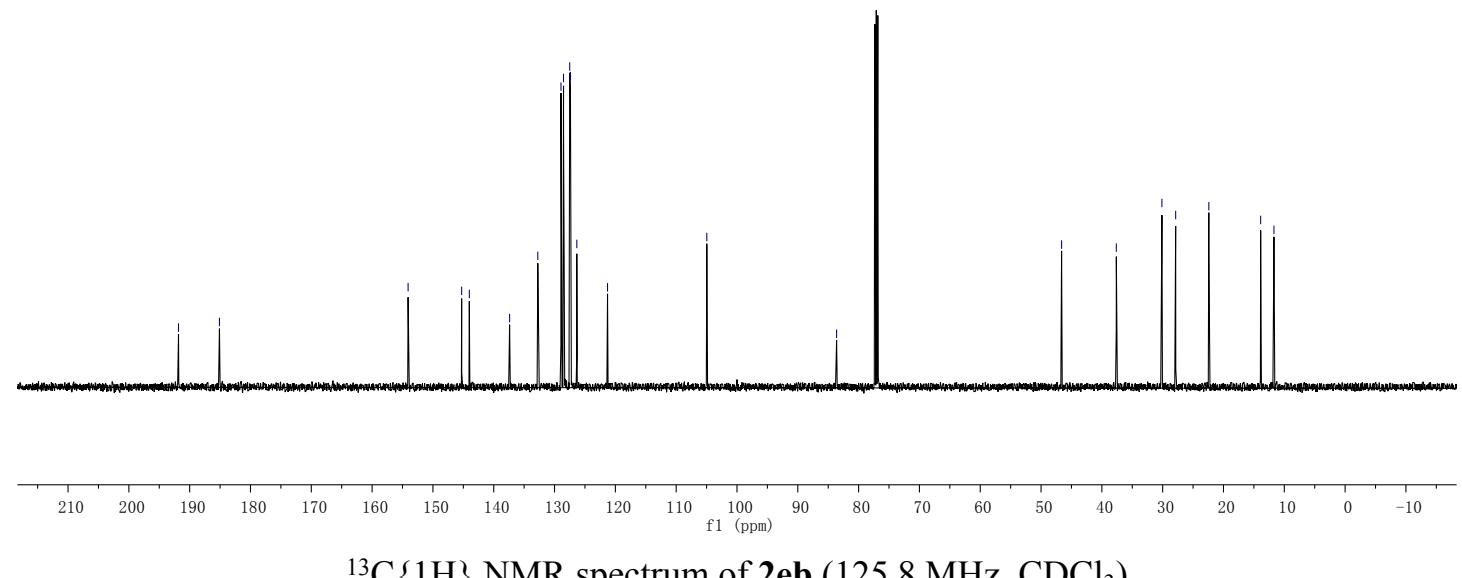

${ }^{13} \mathrm{C}\{1 \mathrm{H}\}$ NMR spectrum of $\mathbf{2 e b}\left(125.8 \mathrm{MHz}, \mathrm{CDCl}_{3}\right)$ 
<smiles>Cc1oc(C(C)(C)C)cc1C(CC(=O)C(=N)c1ccccc1)c1ccccc1</smiles>

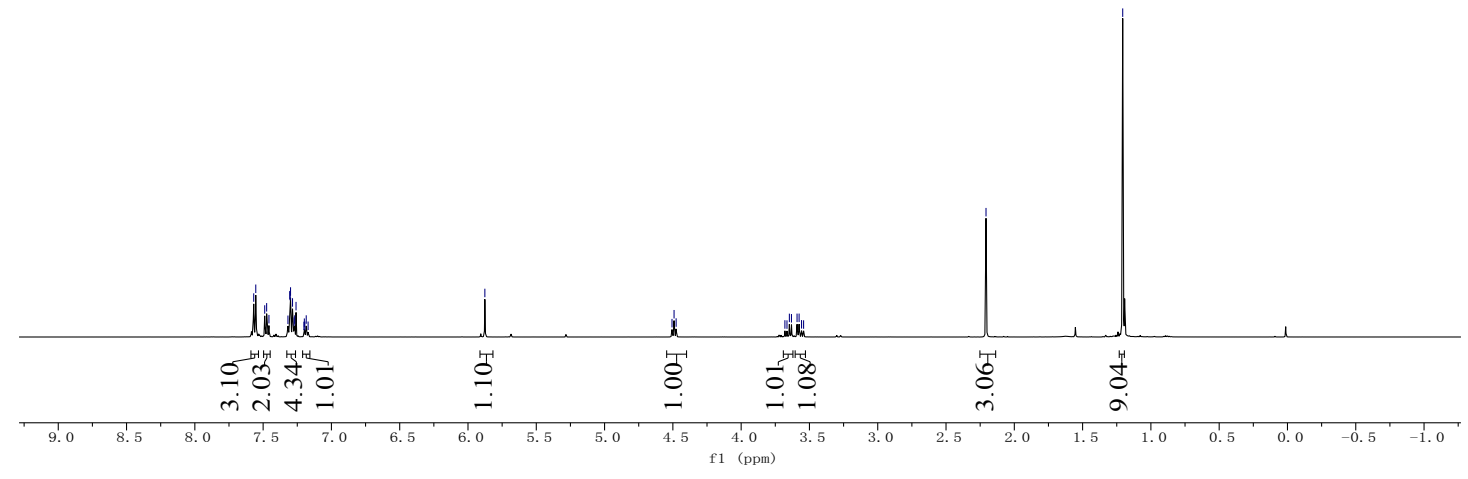

${ }^{1} \mathrm{H}$ NMR spectrum of $\mathbf{2} \mathbf{f b}\left(500 \mathrm{MHz}, \mathrm{CDCl}_{3}\right)$

曈重

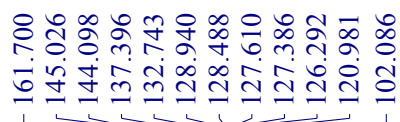

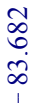

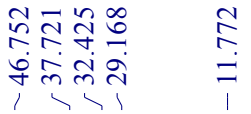
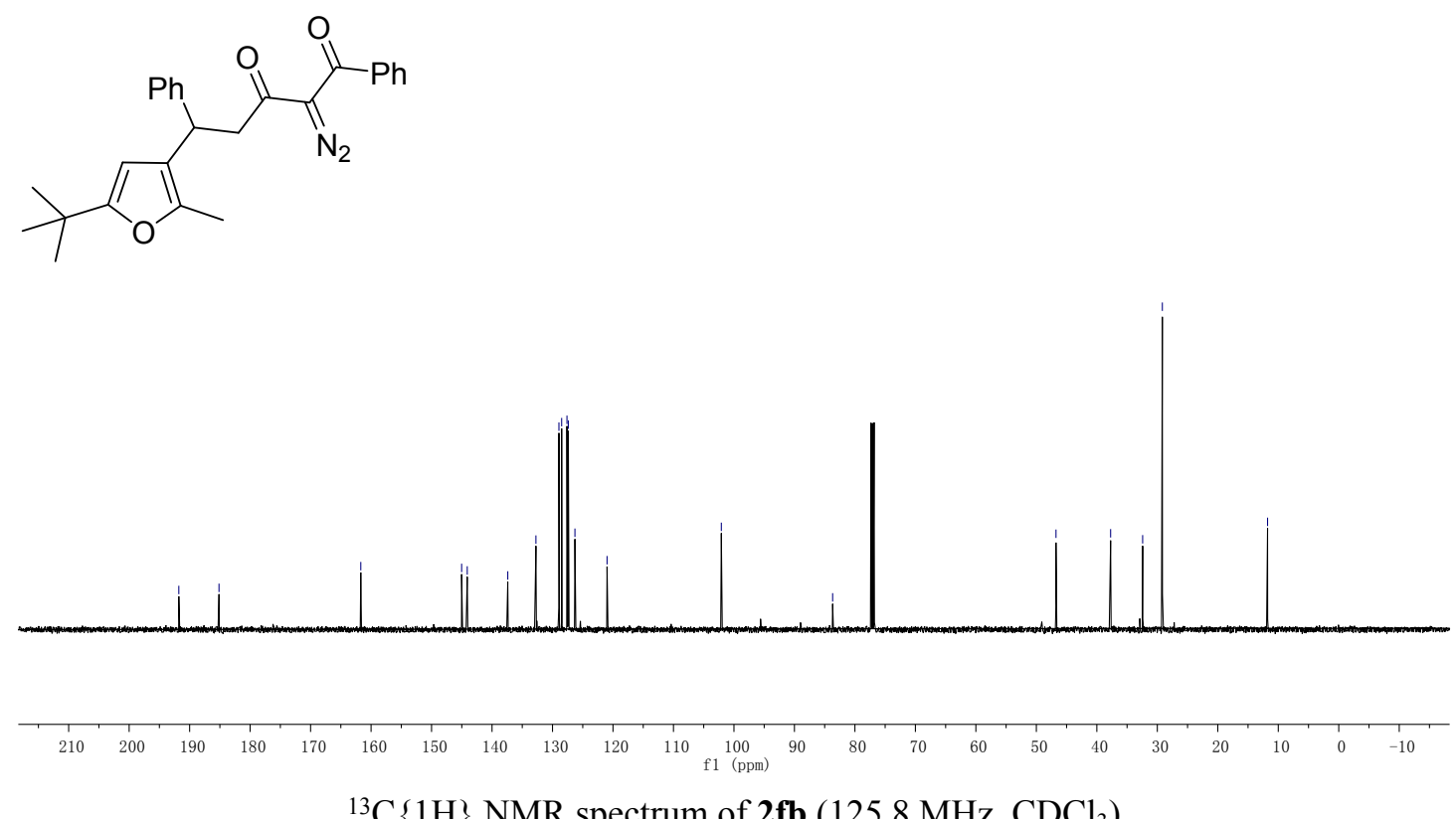

${ }^{13} \mathrm{C}\{1 \mathrm{H}\}$ NMR spectrum of $\mathbf{2} \mathbf{f b}\left(125.8 \mathrm{MHz}, \mathrm{CDCl}_{3}\right)$ 
<smiles>Cc1occc1C(CC(=O)C(=N)C(=O)c1ccccc1)c1ccccc1</smiles>

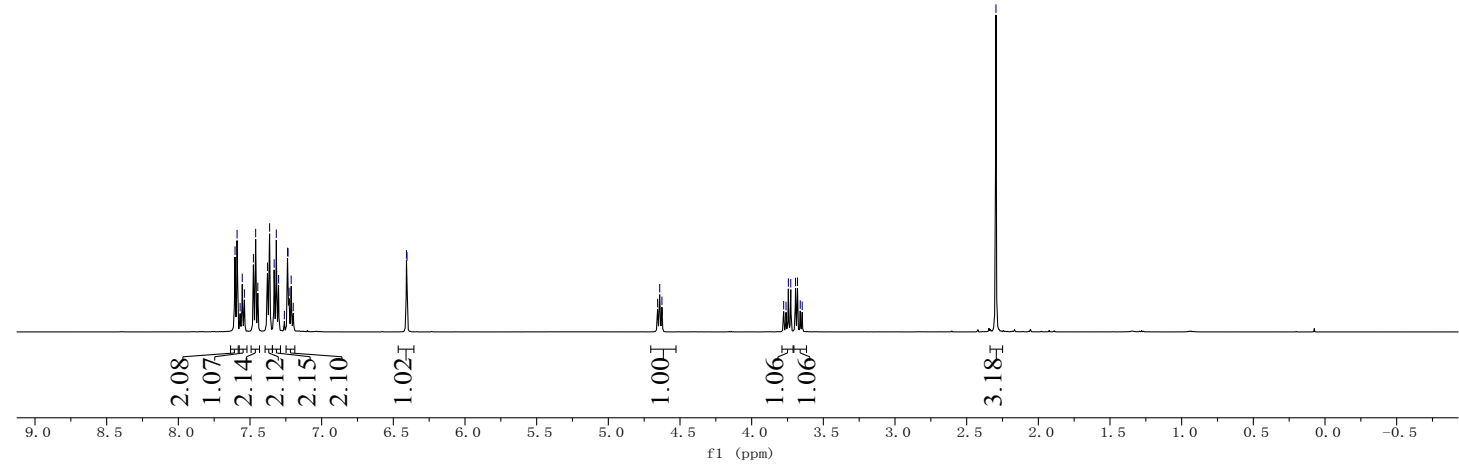

${ }^{1} \mathrm{H}$ NMR spectrum of $2 \mathbf{g b}\left(500 \mathrm{MHz}, \mathrm{CDCl}_{3}\right)$

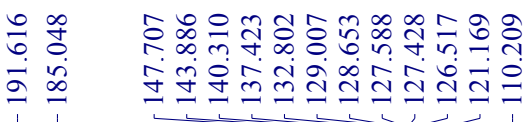

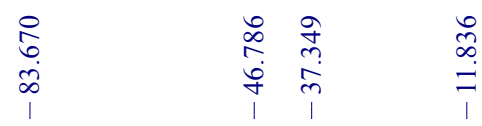<smiles>Cc1occc1C(CC(=O)C(=N)C(=O)c1ccccc1)c1ccccc1</smiles>
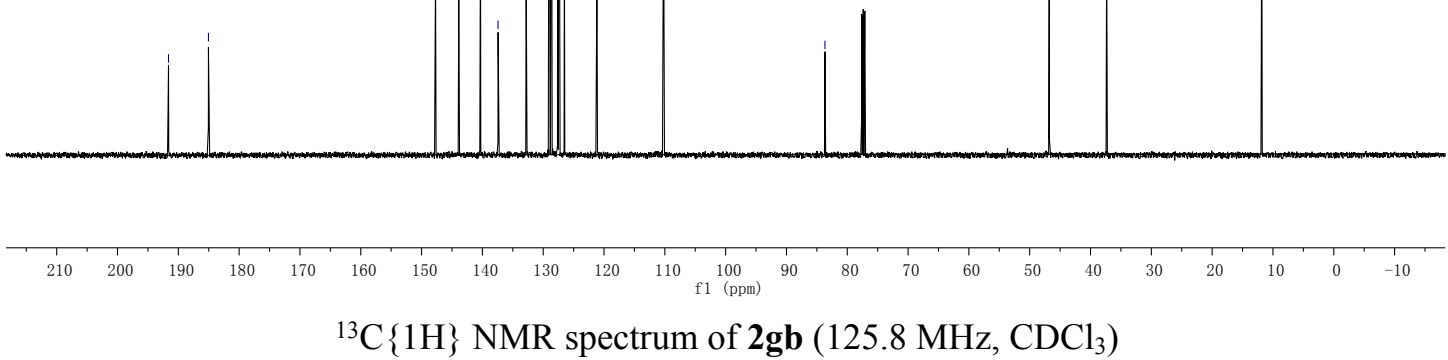
<smiles>CCOC(=O)C1=C(C)/C(=C\C(=O)c2ccccc2)C(c2ccccc2)CC1=O</smiles>

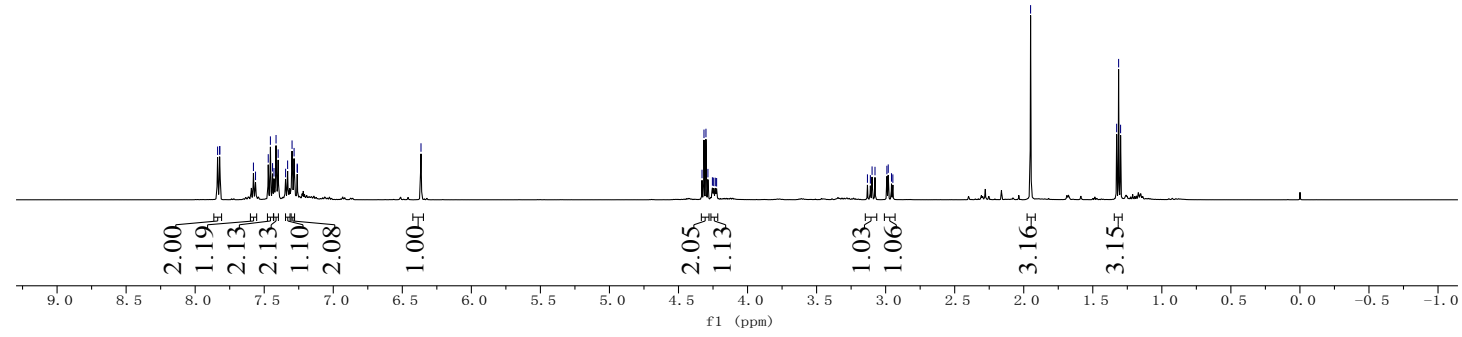

${ }^{1} \mathrm{H}$ NMR spectrum of $\mathbf{3 a a}\left(500 \mathrm{MHz}, \mathrm{CDCl}_{3}\right)$<smiles>CCOC(=O)C1=C(C)/C(=C\C(=O)c2ccccc2)C(c2ccccc2)CC1=O</smiles>
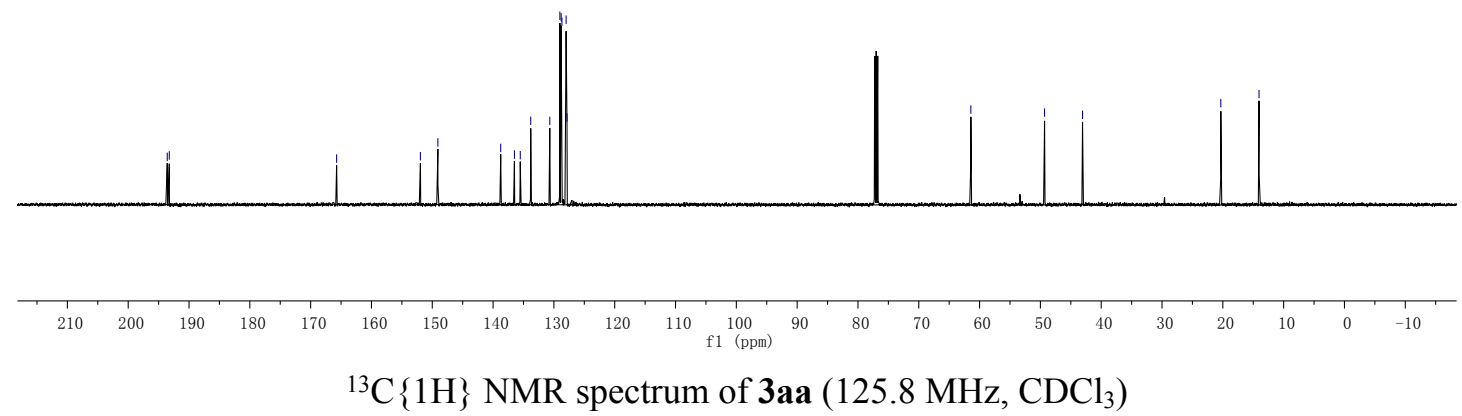
<smiles>CCOC(=O)C1=C(C)C(=CC(=O)c2ccc(F)cc2)C(c2ccccc2)CC1=O</smiles>

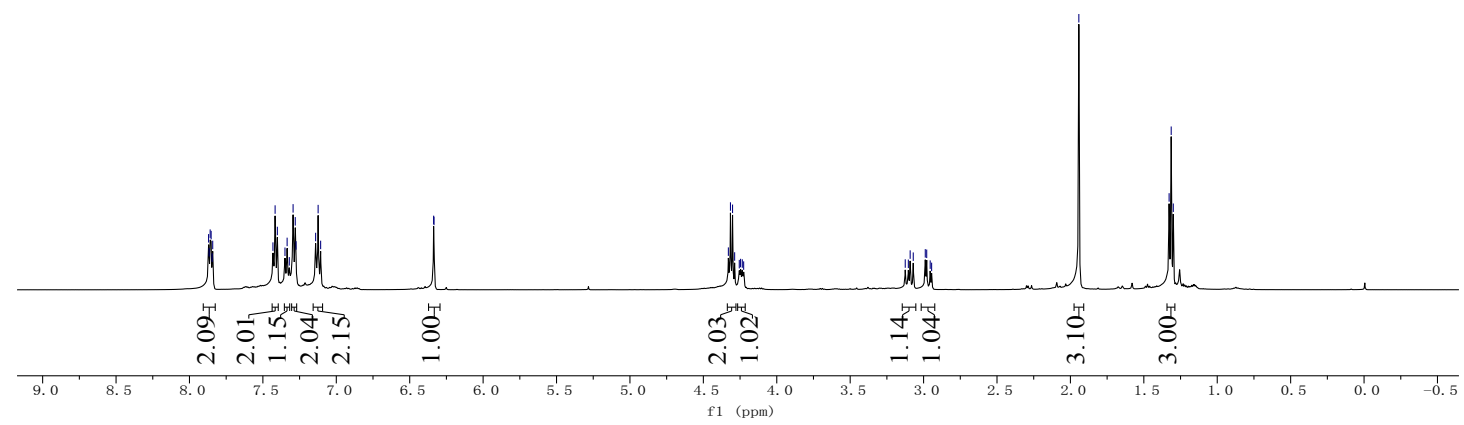

${ }^{1} \mathrm{H}$ NMR spectrum of $\mathbf{3 f a}\left(500 \mathrm{MHz}, \mathrm{CDCl}_{3}\right)$

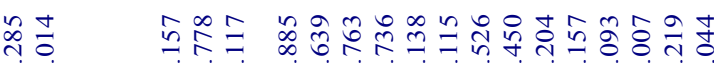

ڤ̆

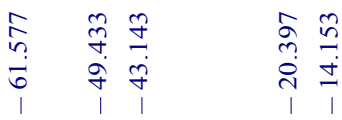<smiles>CCOC(=O)C1=C(C)C(=CC(=O)c2ccc(F)cc2)C(c2ccccc2)CC1=O</smiles>
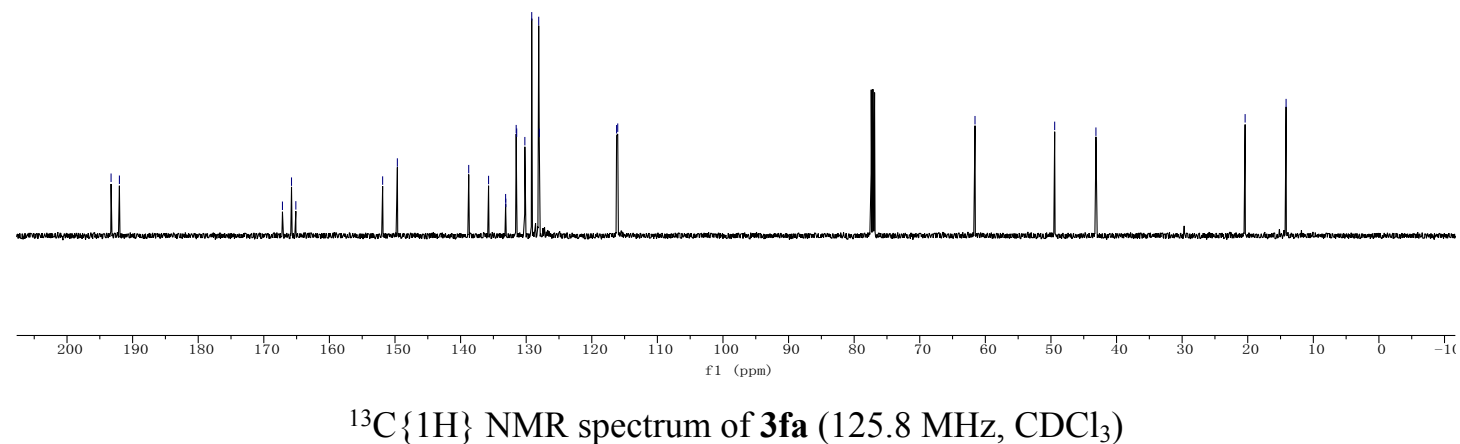

${ }^{13} \mathrm{C}\{1 \mathrm{H}\}$ NMR spectrum of $\mathbf{3 f a}\left(125.8 \mathrm{MHz}, \mathrm{CDCl}_{3}\right)$ 

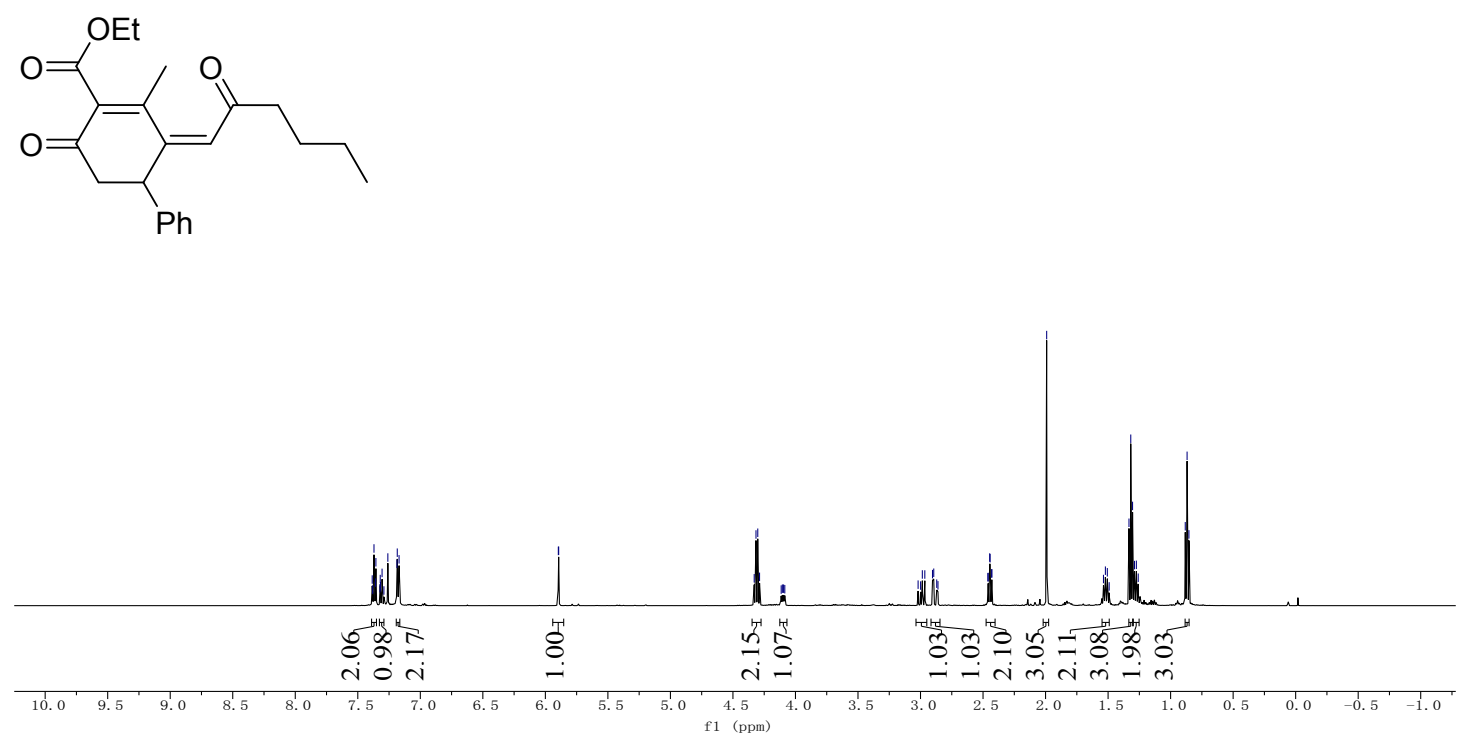

${ }^{1} \mathrm{H}$ NMR spectrum of 3 ha $\left(500 \mathrm{MHz}, \mathrm{CDCl}_{3}\right)$

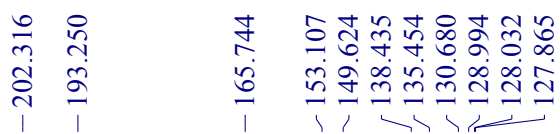

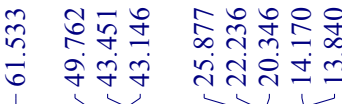
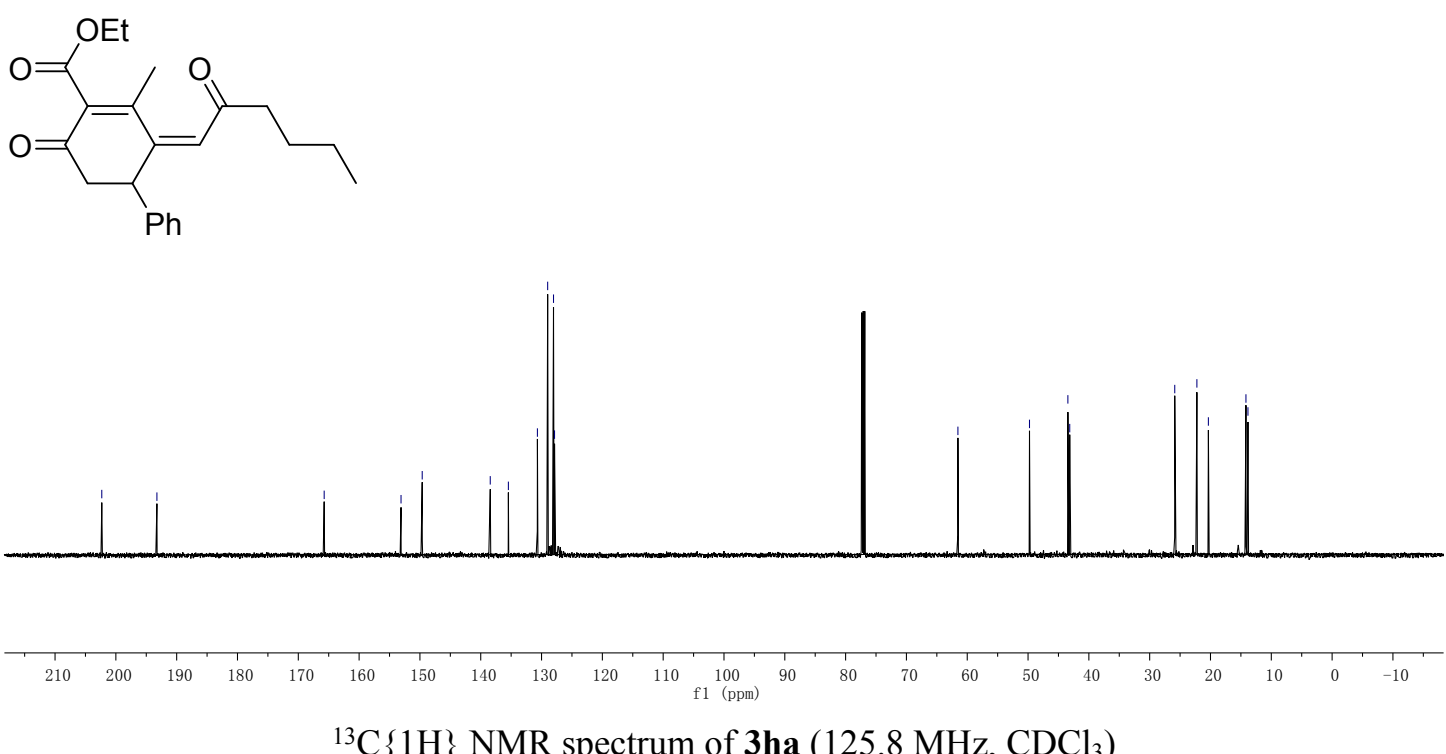

${ }^{13} \mathrm{C}\{1 \mathrm{H}\} \mathrm{NMR}$ spectrum of $\mathbf{3 h a}\left(125.8 \mathrm{MHz}, \mathrm{CDCl}_{3}\right)$ 
<smiles>CCOC(=O)C1=C(C)/C(=C\C(=O)C(C)(C)C)C(c2ccccc2)CC1=O</smiles>

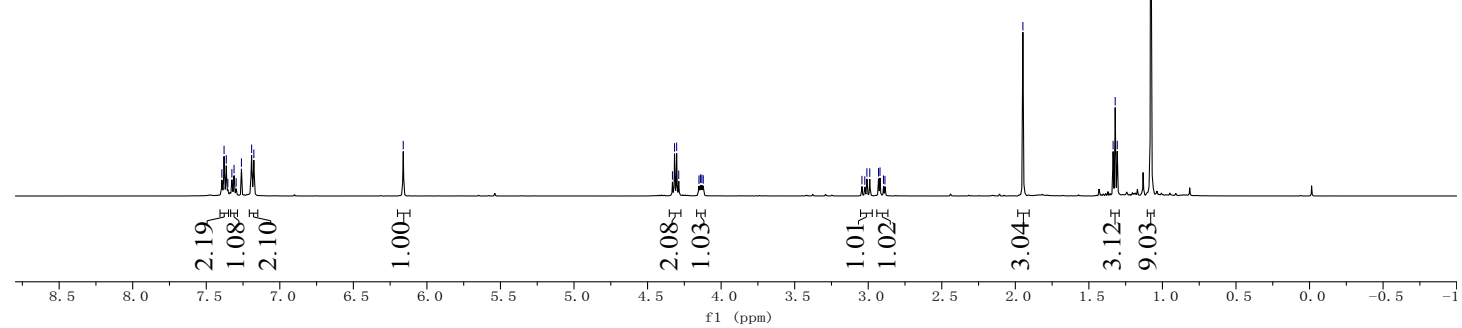

${ }^{1} \mathrm{H}$ NMR spectrum of 3 ia $\left(500 \mathrm{MHz}, \mathrm{CDCl}_{3}\right)$
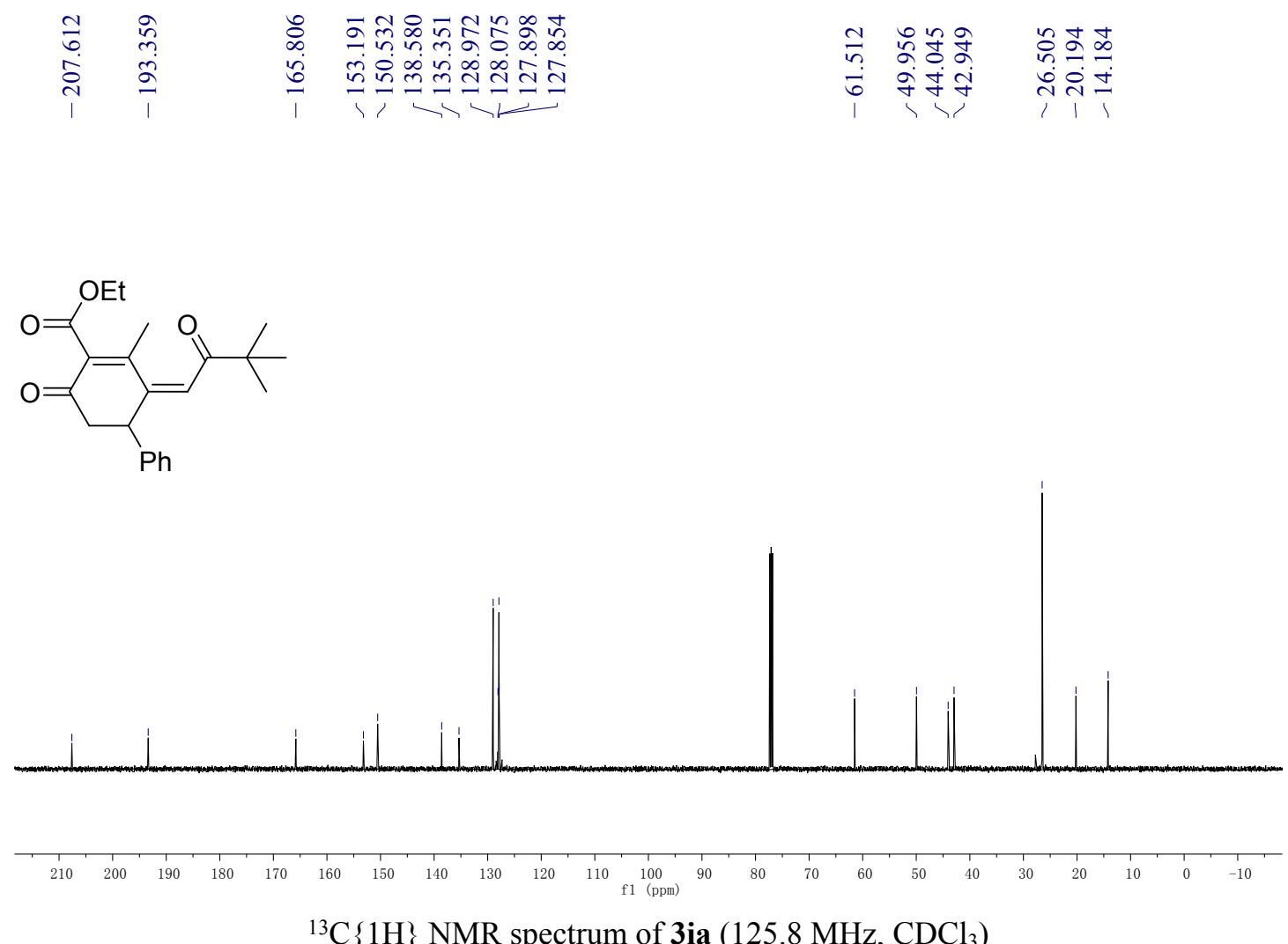

${ }^{13} \mathrm{C}\{1 \mathrm{H}\}$ NMR spectrum of 3 ia $\left(125.8 \mathrm{MHz}, \mathrm{CDCl}_{3}\right)$ 
<smiles>CCOC(=O)C1=C(C)C(=CC=O)C(c2ccccc2)CC1=O</smiles>

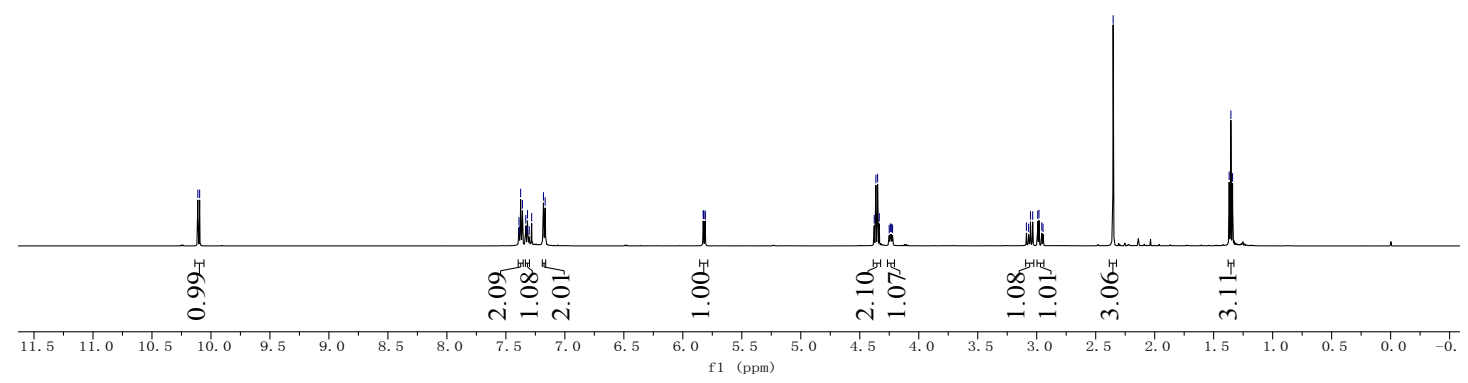

${ }^{1} \mathrm{H}$ NMR spectrum of $\mathbf{3 j a}\left(500 \mathrm{MHz}, \mathrm{CDCl}_{3}\right)$
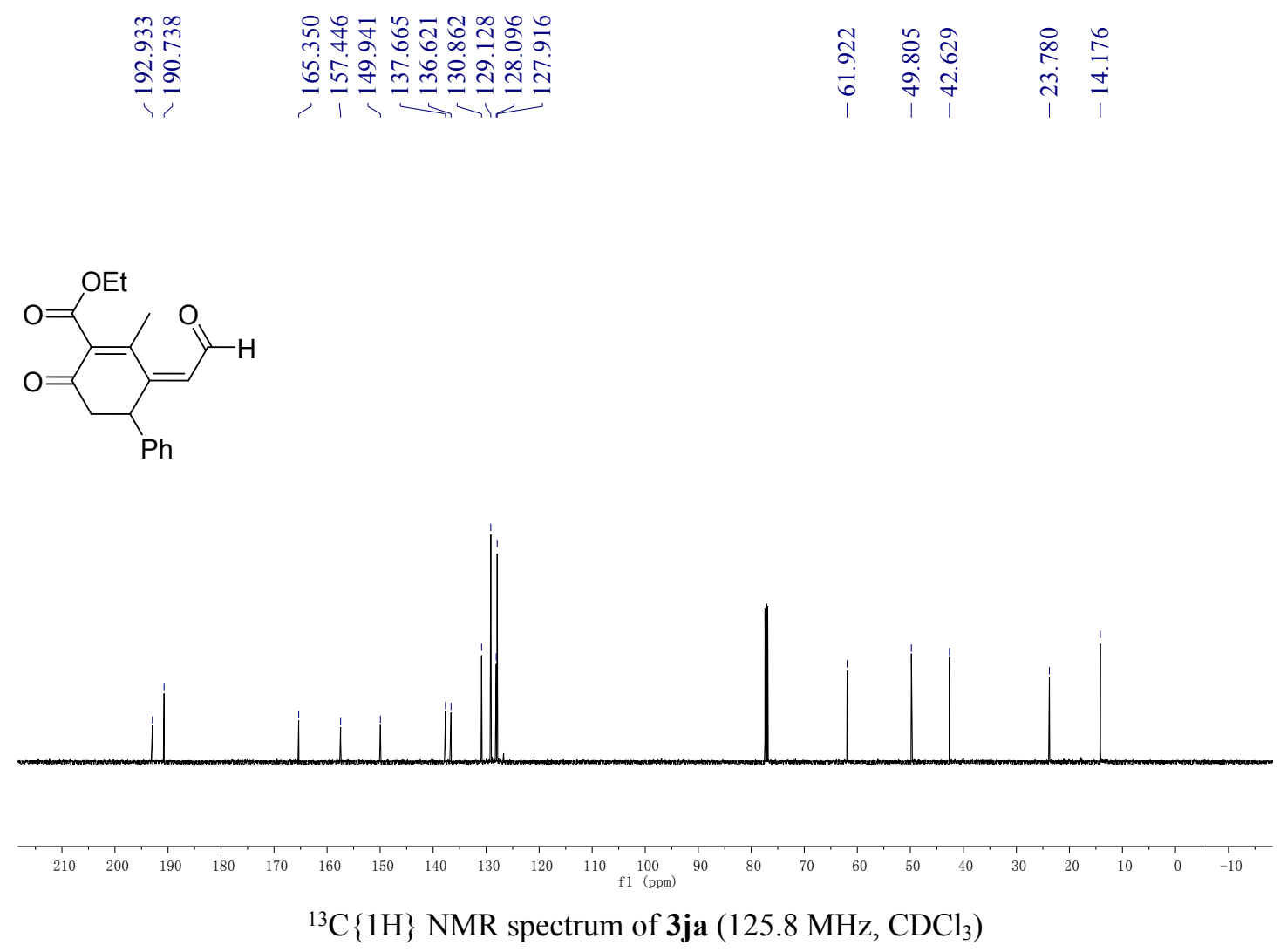

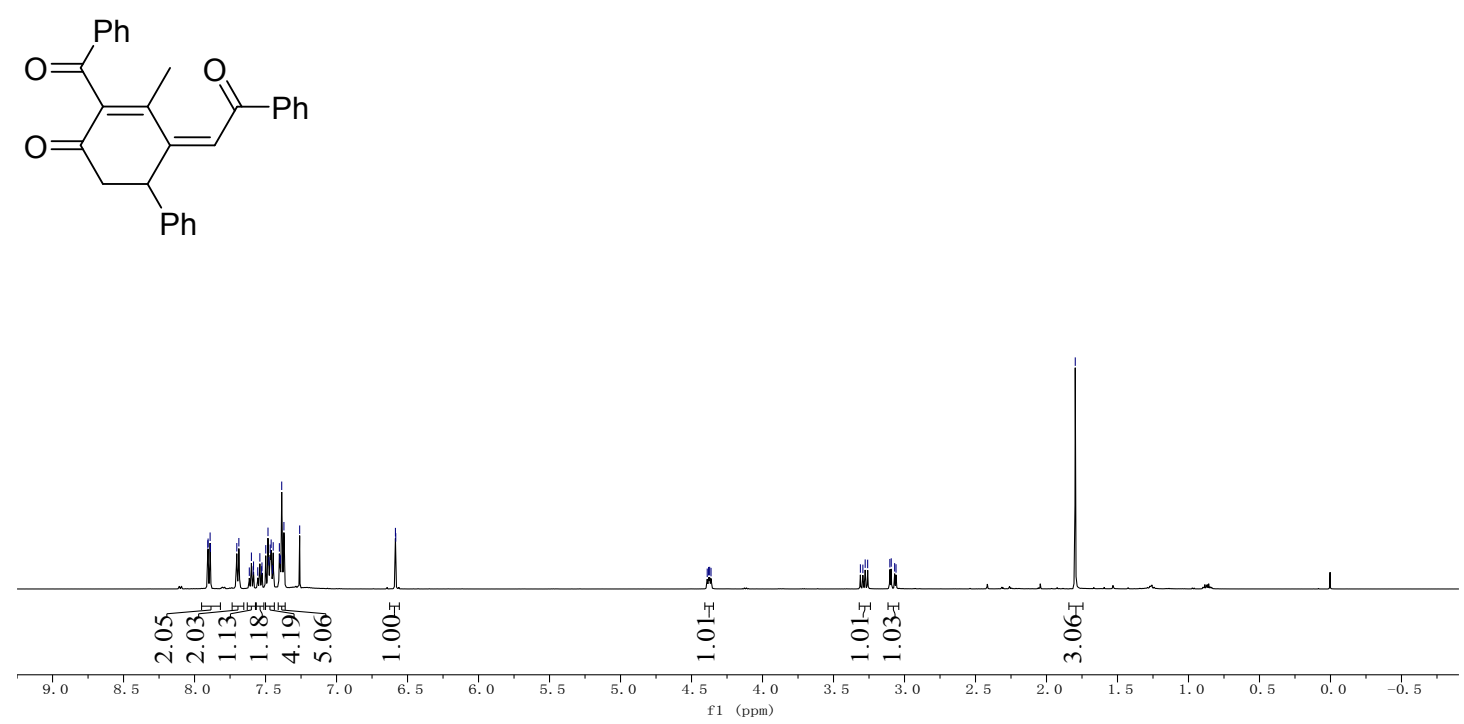

${ }^{1} \mathrm{H}$ NMR spectrum of $\mathbf{3 a b}\left(500 \mathrm{MHz}, \mathrm{CDCl}_{3}\right)$

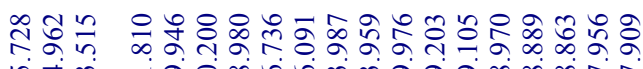

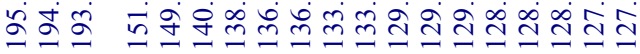

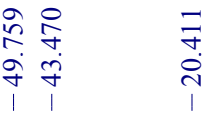<smiles>CC1=C(C(=O)c2ccccc2)C(=O)CC(c2ccccc2)C1=Cc1ccccc1</smiles>
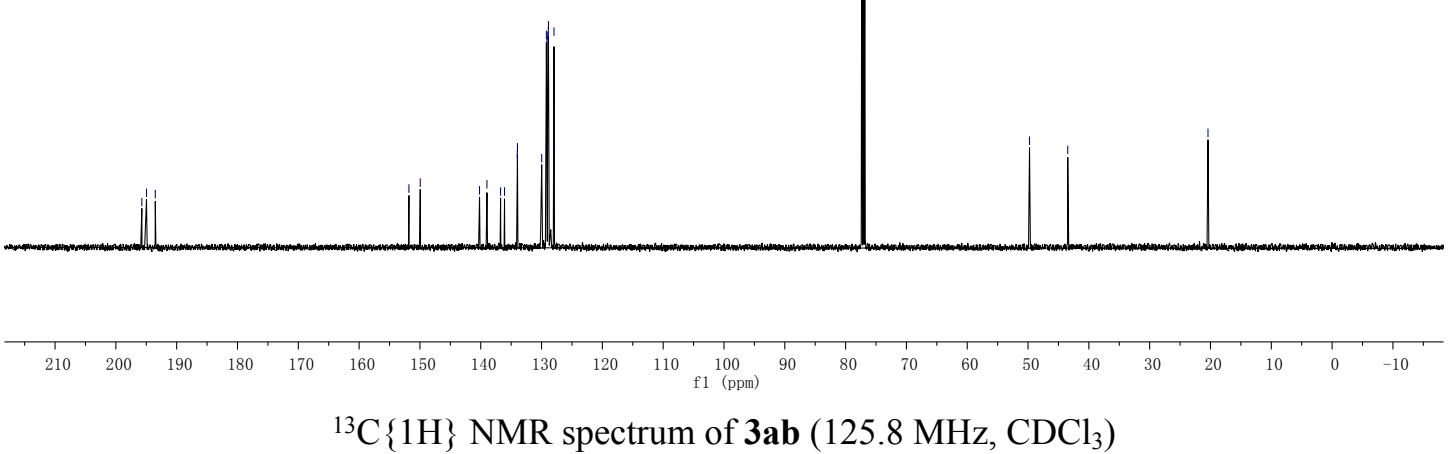
<smiles>CC1=C(C(=O)c2ccccc2)C(=O)CC(c2ccccc2)C1=CC(=O)c1ccc(C)cc1</smiles>

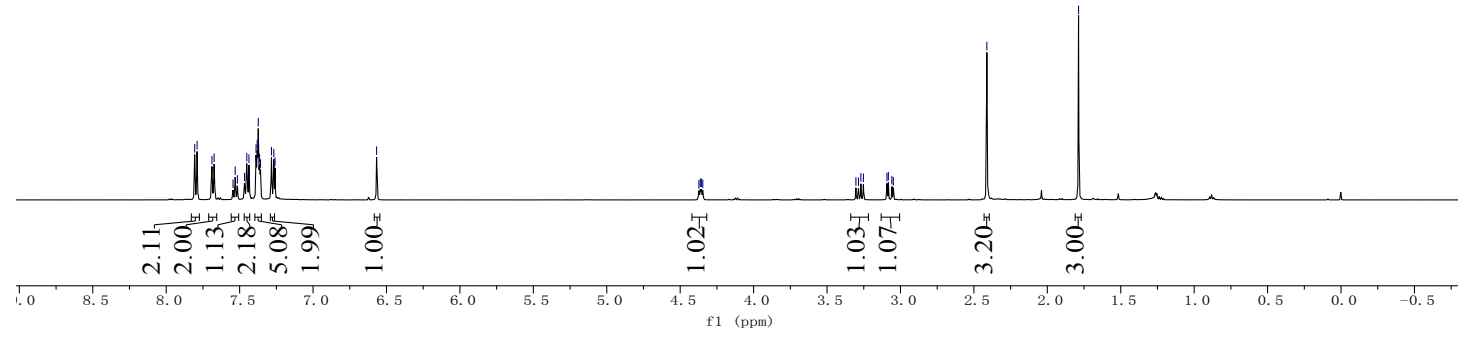

${ }^{1} \mathrm{H}$ NMR spectrum of $\mathbf{3 b b}\left(500 \mathrm{MHz}, \mathrm{CDCl}_{3}\right)$<smiles>CC1=C(C(=O)c2ccccc2)C(=O)CC(c2ccccc2)C1=CC(=O)c1ccc(C)cc1</smiles>
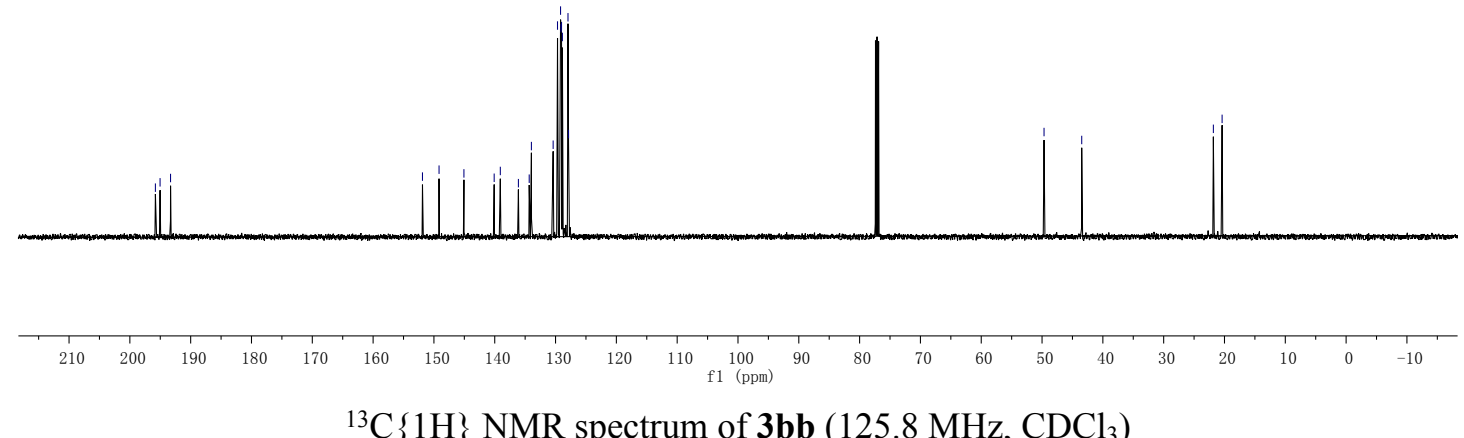

${ }^{13} \mathrm{C}\{1 \mathrm{H}\}$ NMR spectrum of $\mathbf{3 b b}\left(125.8 \mathrm{MHz}, \mathrm{CDCl}_{3}\right)$ 
<smiles>C=C1C(C)=C(C(=O)c2ccccc2)C(=O)CC1c1ccccc1</smiles>

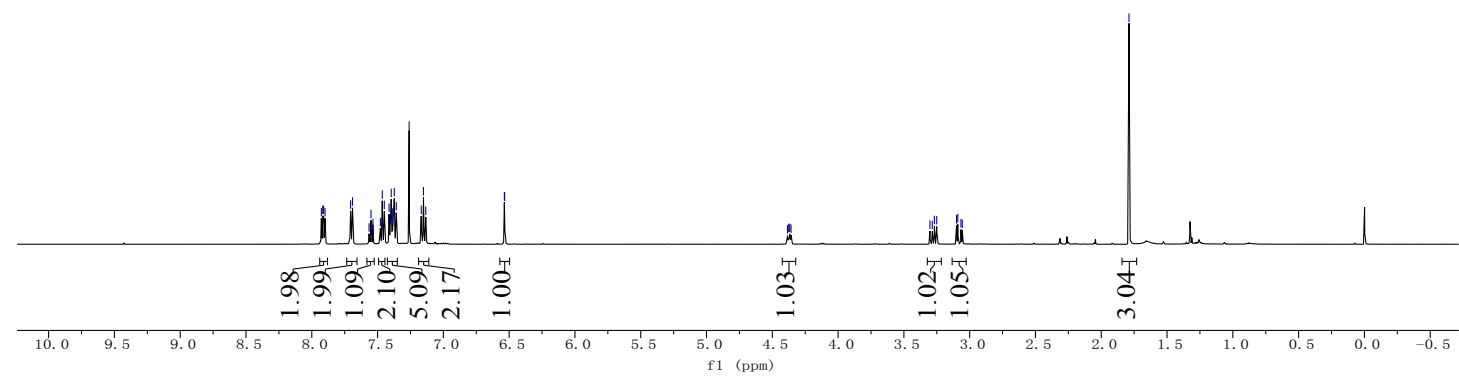

${ }^{1} \mathrm{H}$ NMR spectrum of $\mathbf{3 c b}\left(500 \mathrm{MHz}, \mathrm{CDCl}_{3}\right)$

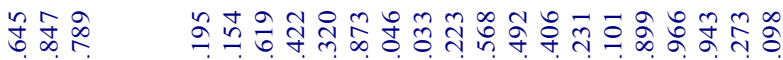

尚方示

(

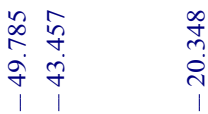<smiles>CC1=C(C(=O)c2ccccc2)C(=O)CC(c2ccccc2)C1=CC(=O)c1ccc(F)cc1</smiles>
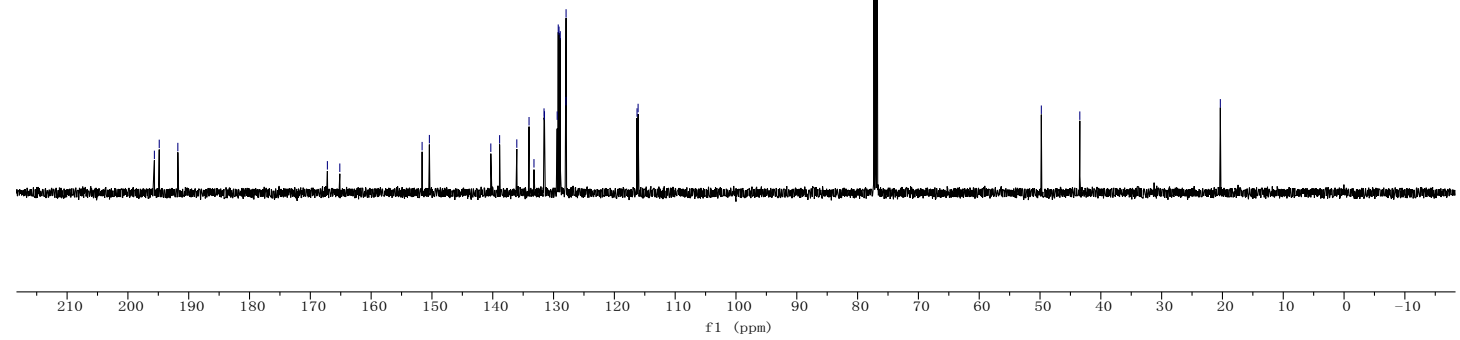

${ }^{13} \mathrm{C}\{1 \mathrm{H}\}$ NMR spectrum of $\mathbf{3 c b}\left(125.8 \mathrm{MHz}, \mathrm{CDCl}_{3}\right)$ 

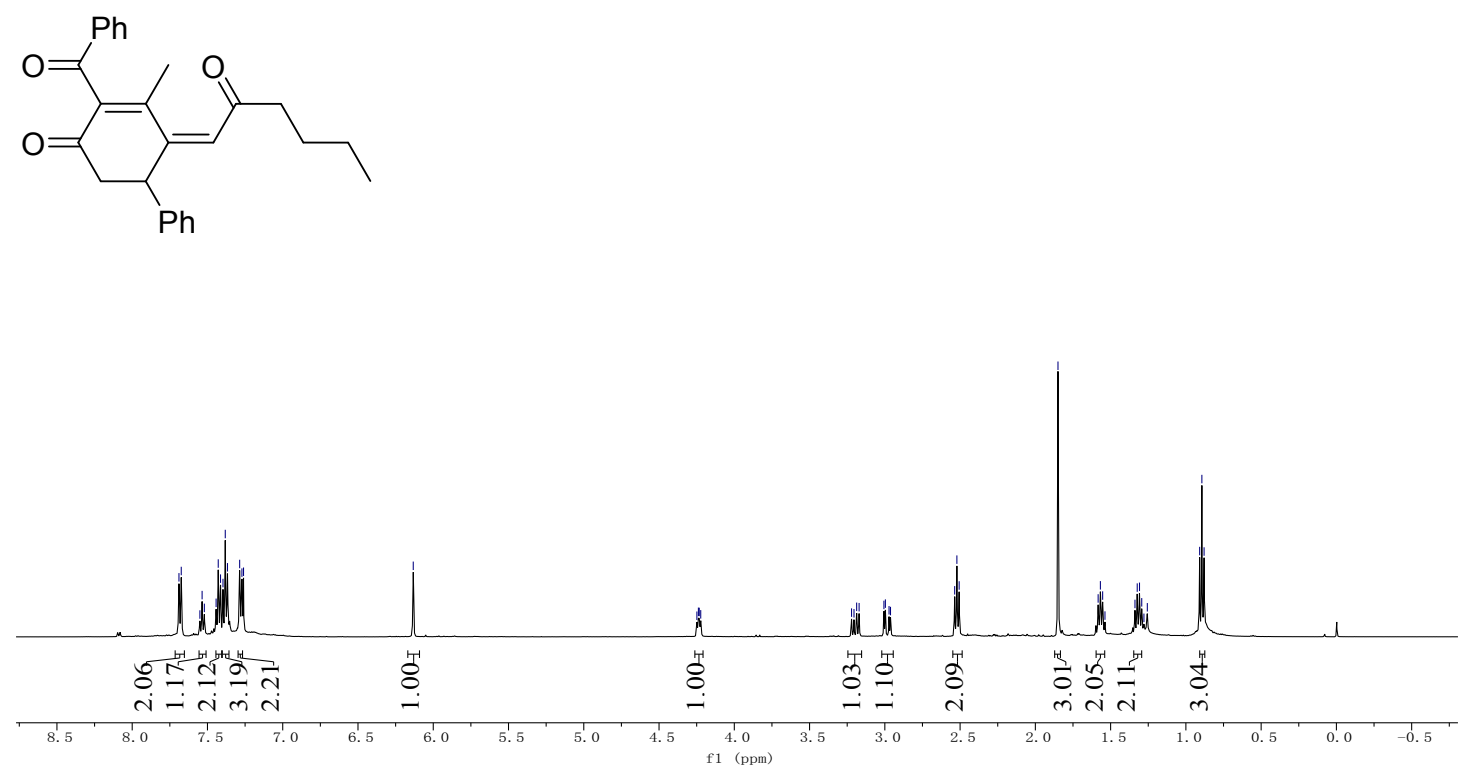

${ }^{1} \mathrm{H}$ NMR spectrum of $\mathbf{3 e b}\left(500 \mathrm{MHz}, \mathrm{CDCl}_{3}\right)$

\section{क $\overline{\mathrm{A}}$}

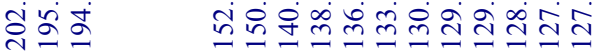
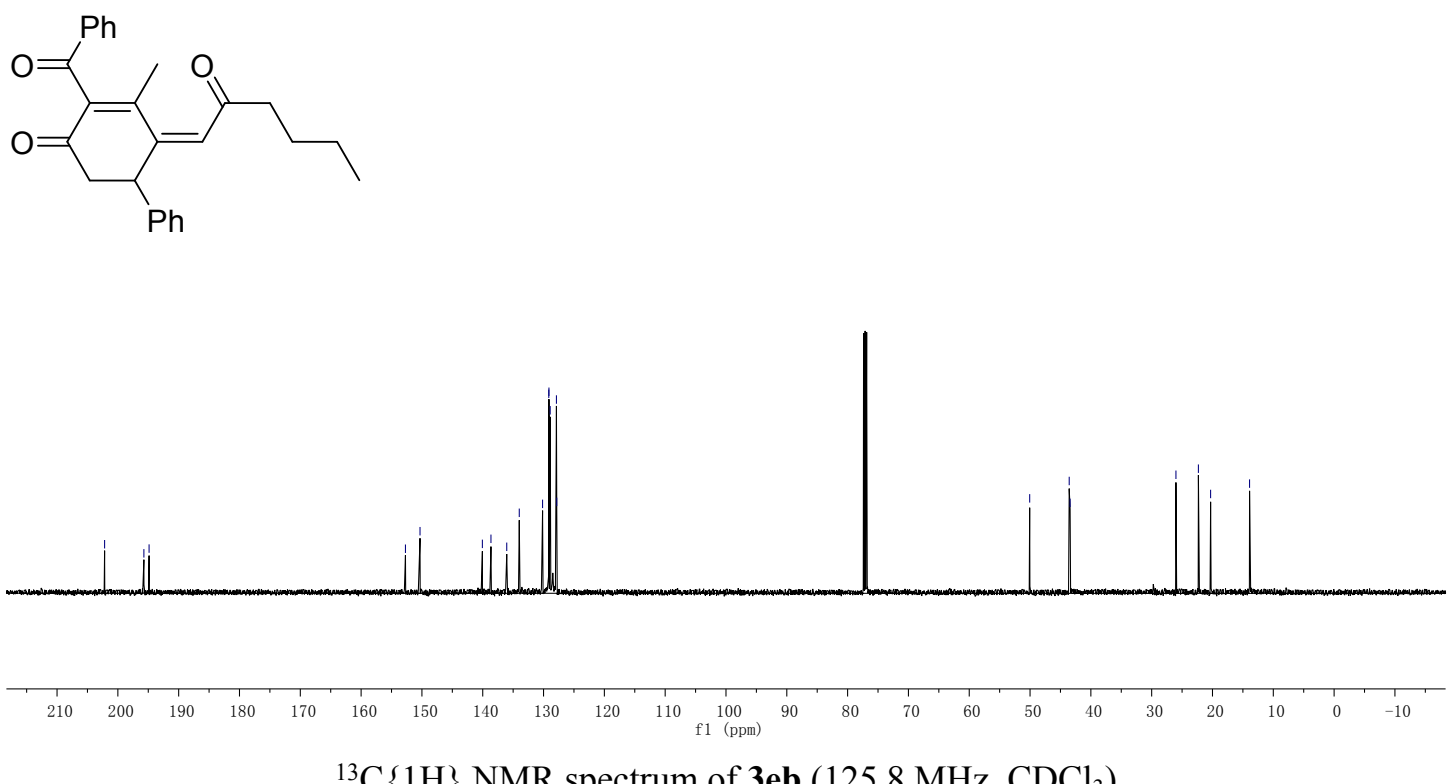

${ }^{13} \mathrm{C}\{1 \mathrm{H}\} \mathrm{NMR}$ spectrum of $\mathbf{3 e b}\left(125.8 \mathrm{MHz}, \mathrm{CDCl}_{3}\right)$ 
<smiles>C=C1C(C)=C(C(=O)c2ccccc2)C(=O)CC1c1ccccc1</smiles>

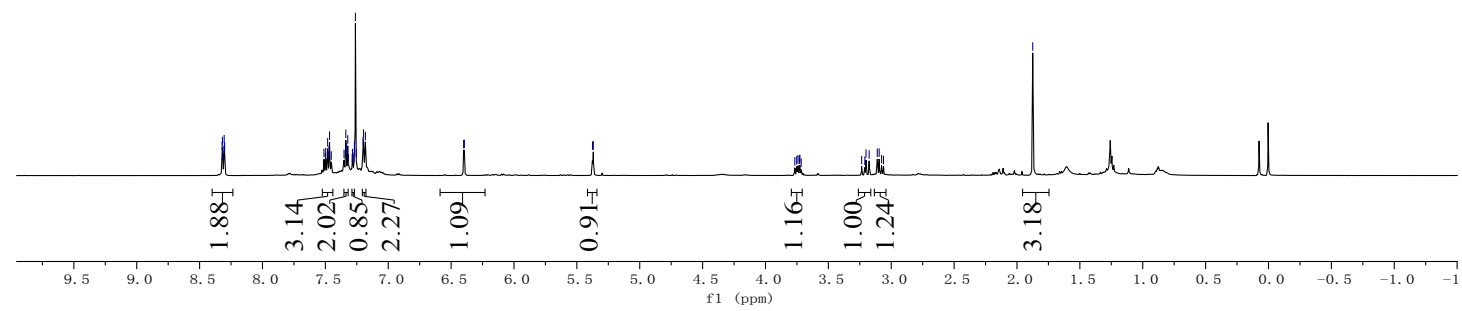

${ }^{1} \mathrm{H}$ NMR spectrum of $\mathbf{3 g b}\left(500 \mathrm{MHz}, \mathrm{CDCl}_{3}\right)$

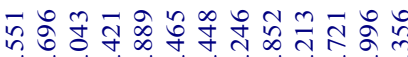

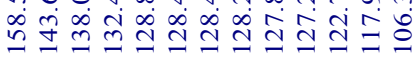

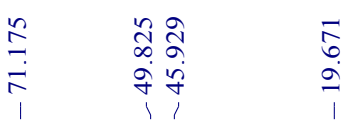<smiles>C=C1C(C)=C(C(=O)c2ccccc2)C(=O)CC1c1ccccc1</smiles>
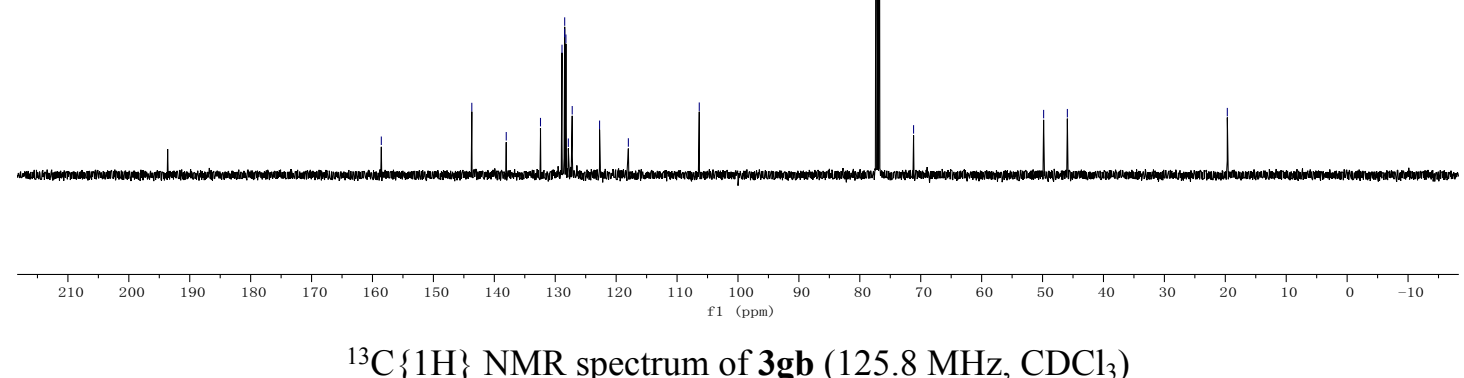

${ }^{13} \mathrm{C}\{1 \mathrm{H}\}$ NMR spectrum of $\mathbf{3 g b}\left(125.8 \mathrm{MHz}, \mathrm{CDCl}_{3}\right)$ 
<smiles>CCOC(=O)c1c(O)cc(-c2ccccc2)c(CC(=O)c2ccccc2)c1C</smiles>

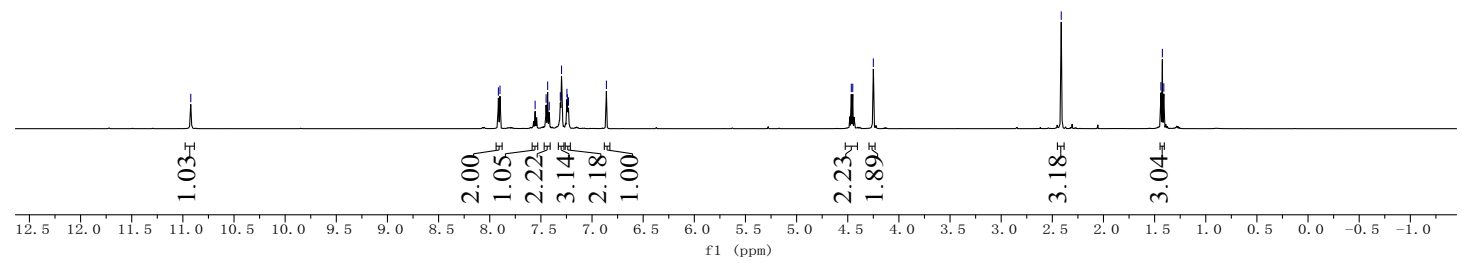

${ }^{1} \mathrm{H}$ NMR spectrum of $\mathbf{4 a a}\left(500 \mathrm{MHz}, \mathrm{CDCl}_{3}\right)$

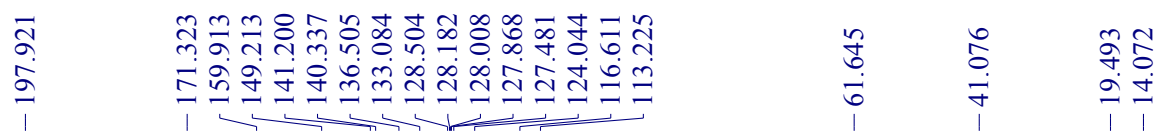<smiles>CCOC(=O)c1c(O)cc(-c2ccccc2)c(CC(=O)c2ccccc2)c1C</smiles>
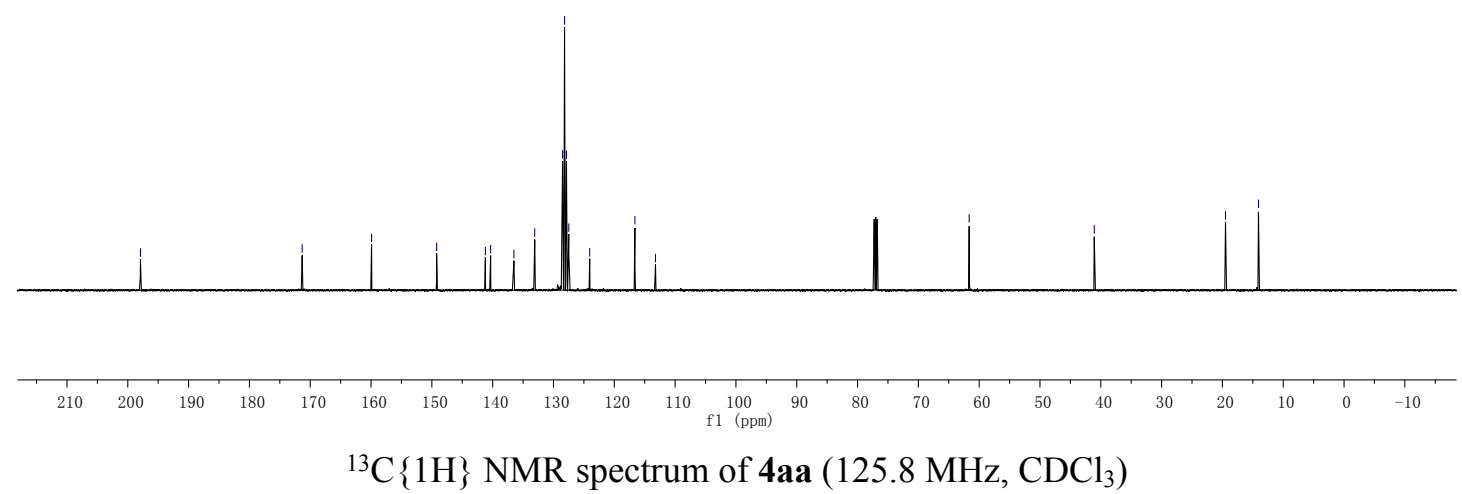


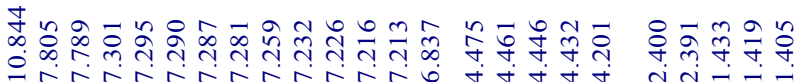

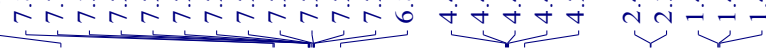
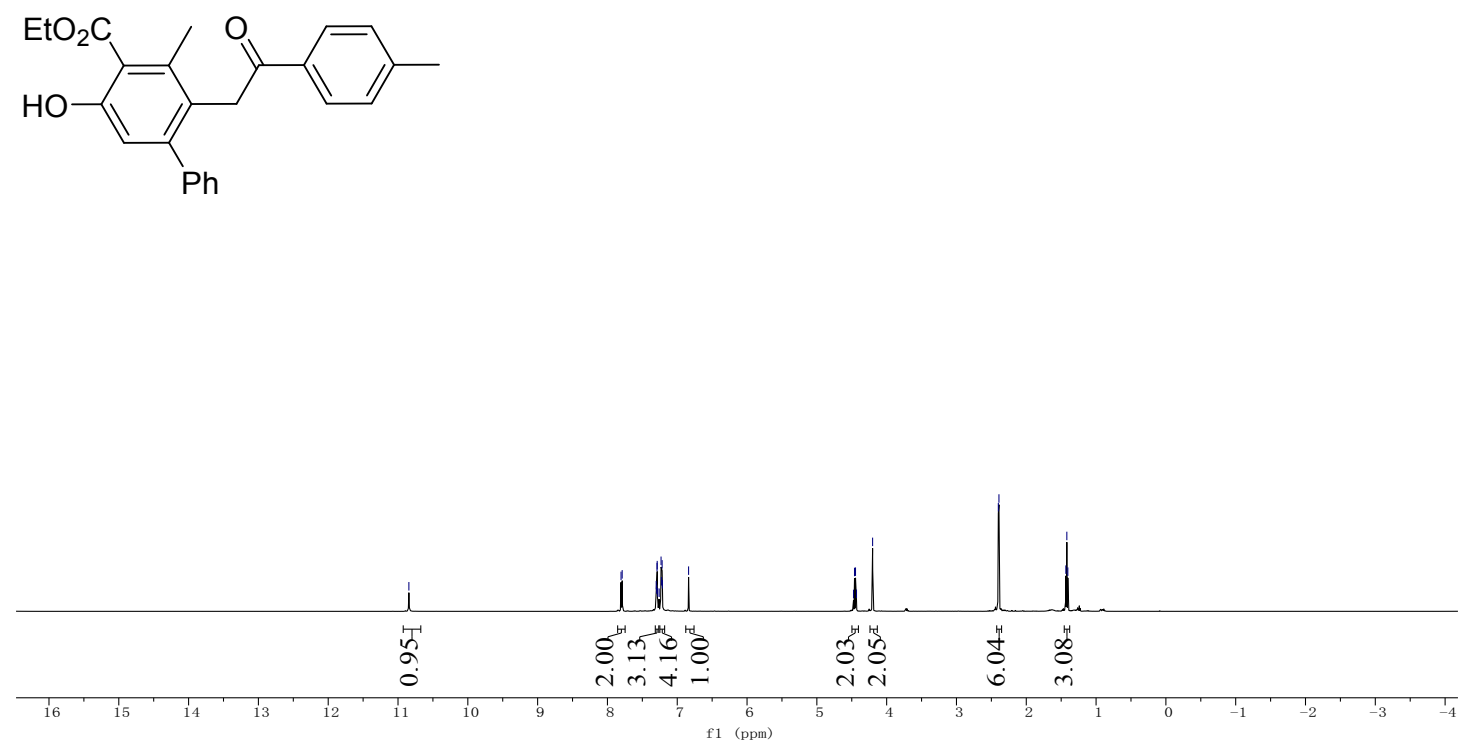

${ }^{1} \mathrm{H}$ NMR spectrum of $\mathbf{4 b a}\left(500 \mathrm{MHz}, \mathrm{CDCl}_{3}\right)$

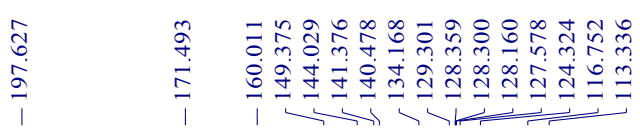

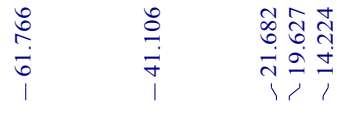
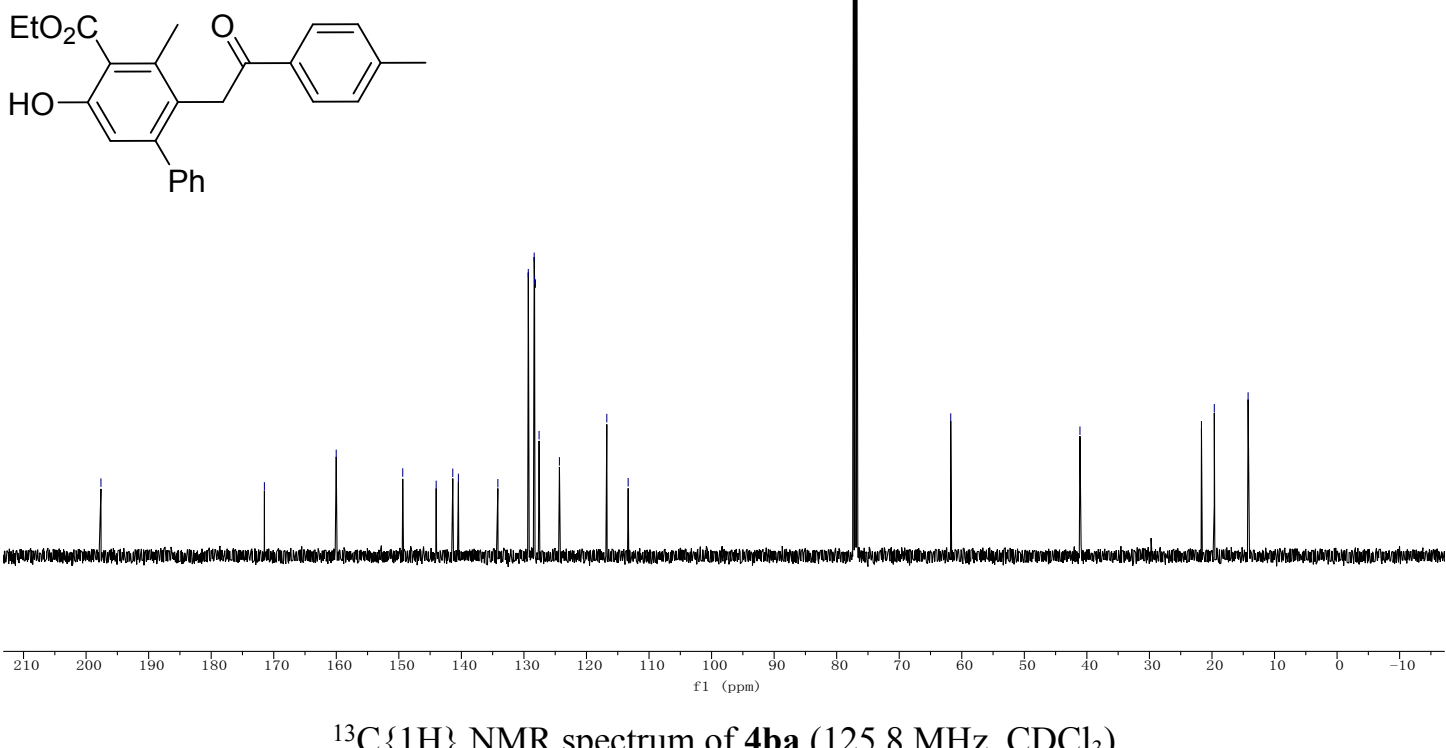

${ }^{13} \mathrm{C}\{1 \mathrm{H}\} \mathrm{NMR}$ spectrum of $\mathbf{4 b a}\left(125.8 \mathrm{MHz}, \mathrm{CDCl}_{3}\right)$ 

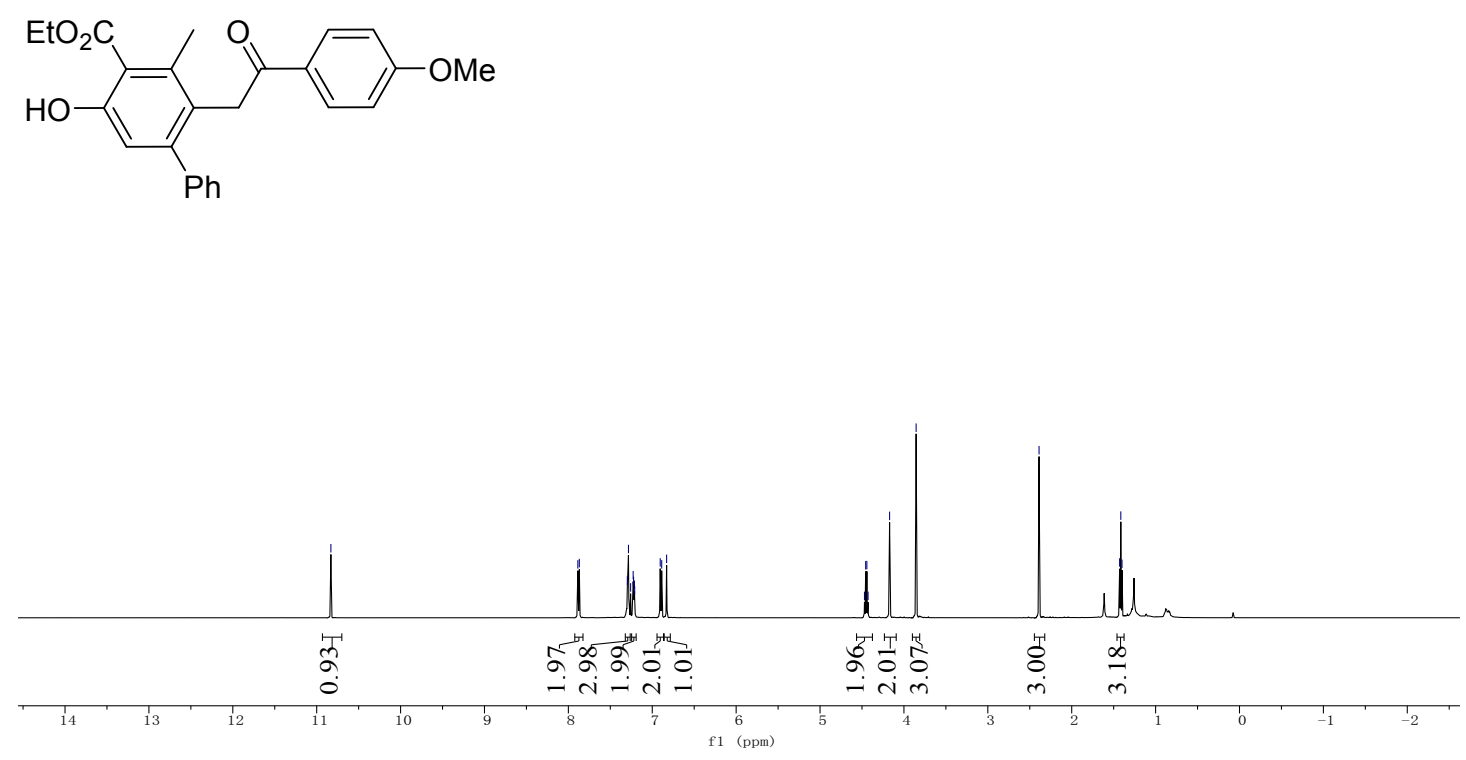

${ }^{1} \mathrm{H}$ NMR spectrum of $\mathbf{4 c a}\left(500 \mathrm{MHz}, \mathrm{CDCl}_{3}\right)$

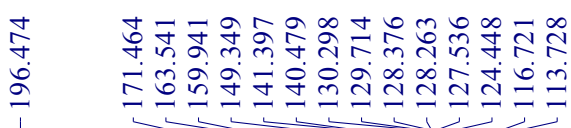

$\vec{n} \mathfrak{g}$

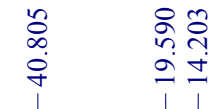
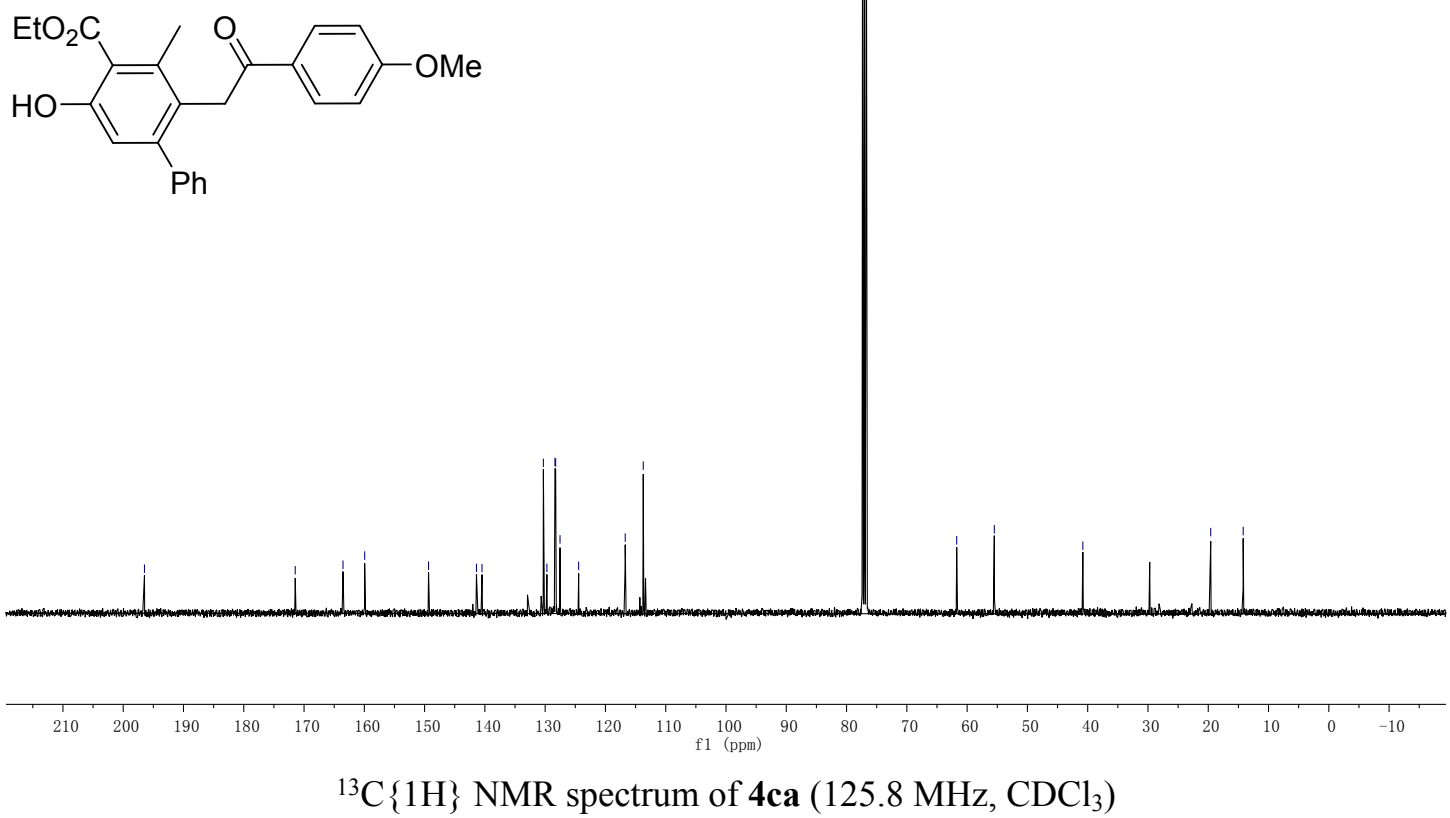

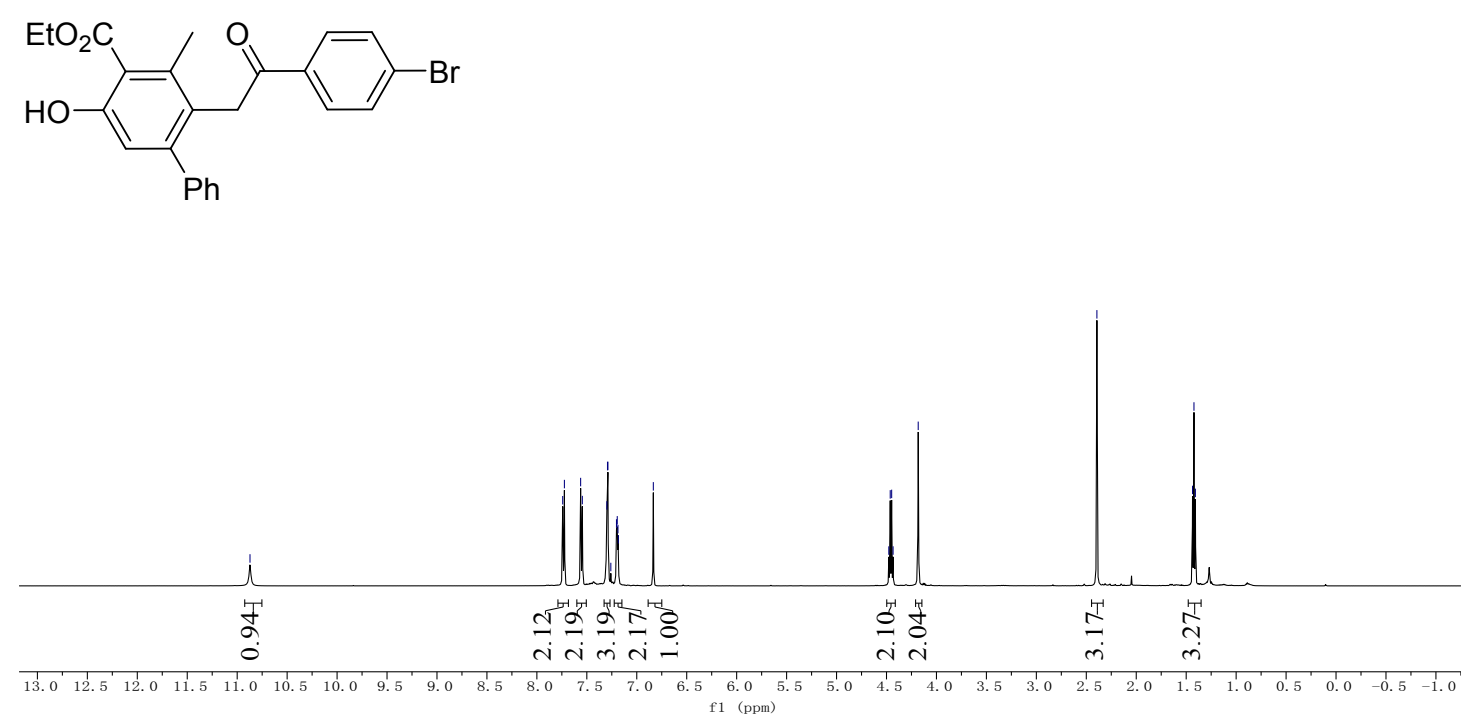

${ }^{1} \mathrm{H}$ NMR spectrum of $\mathbf{4 d a}\left(500 \mathrm{MHz}, \mathrm{CDCl}_{3}\right)$
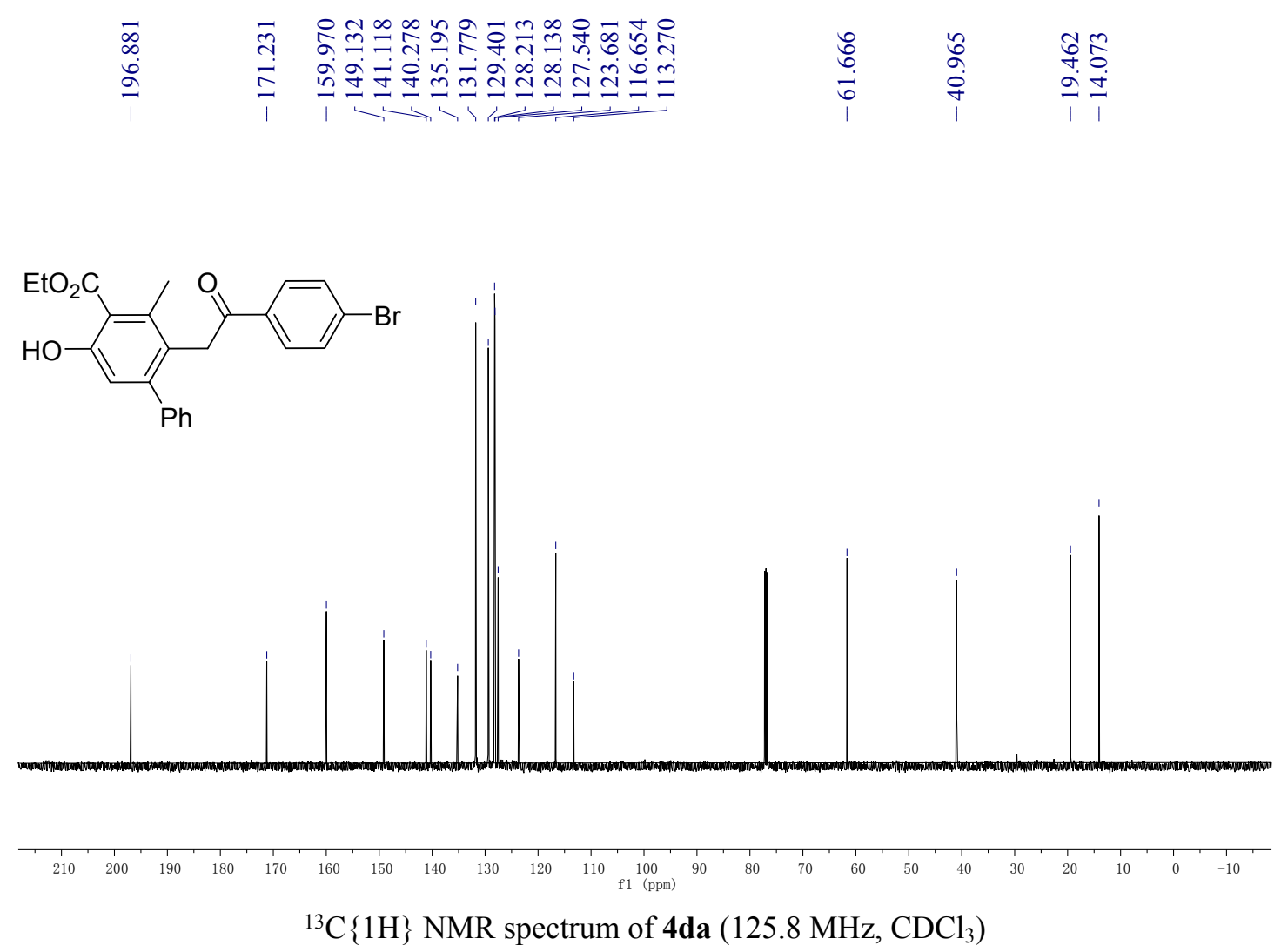

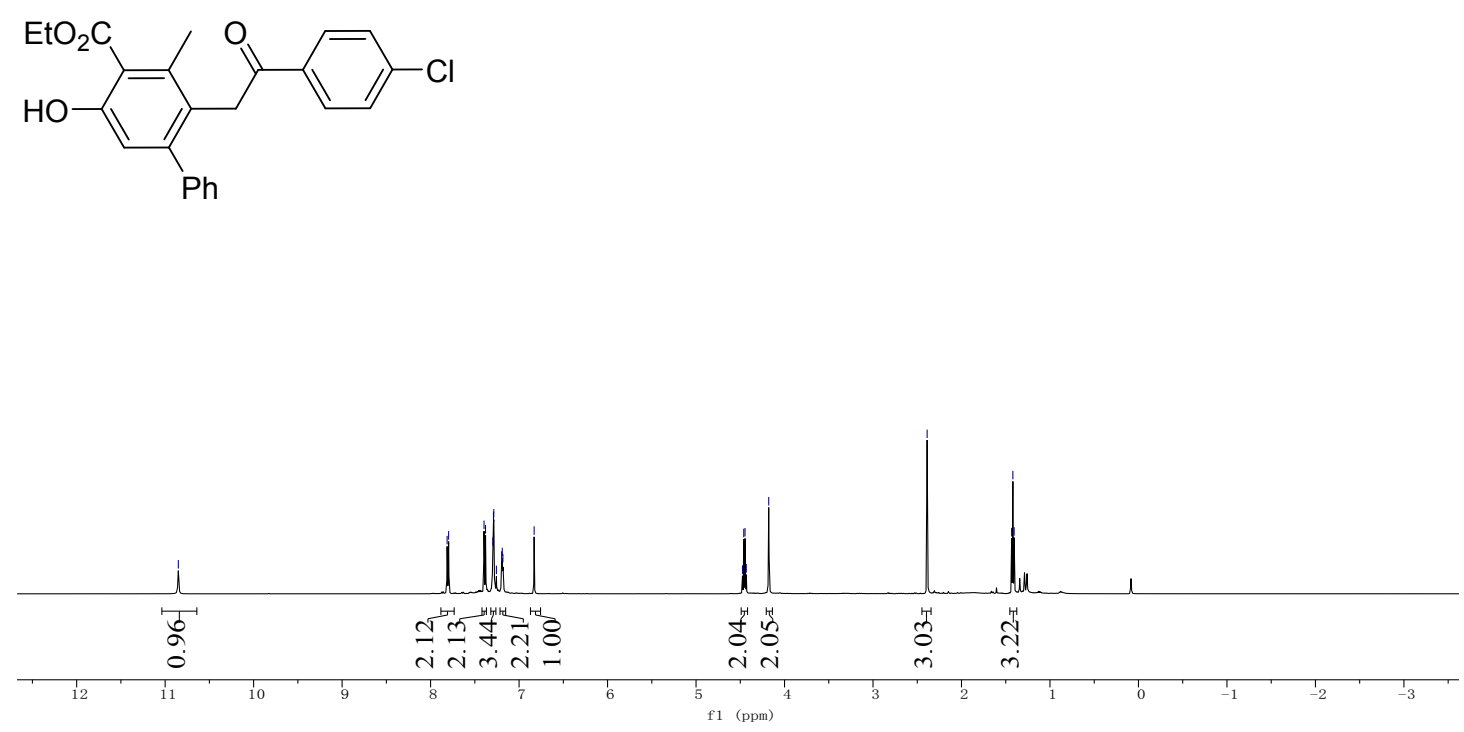

${ }^{1} \mathrm{H}$ NMR spectrum of 4 ea $\left(500 \mathrm{MHz}, \mathrm{CDCl}_{3}\right)$
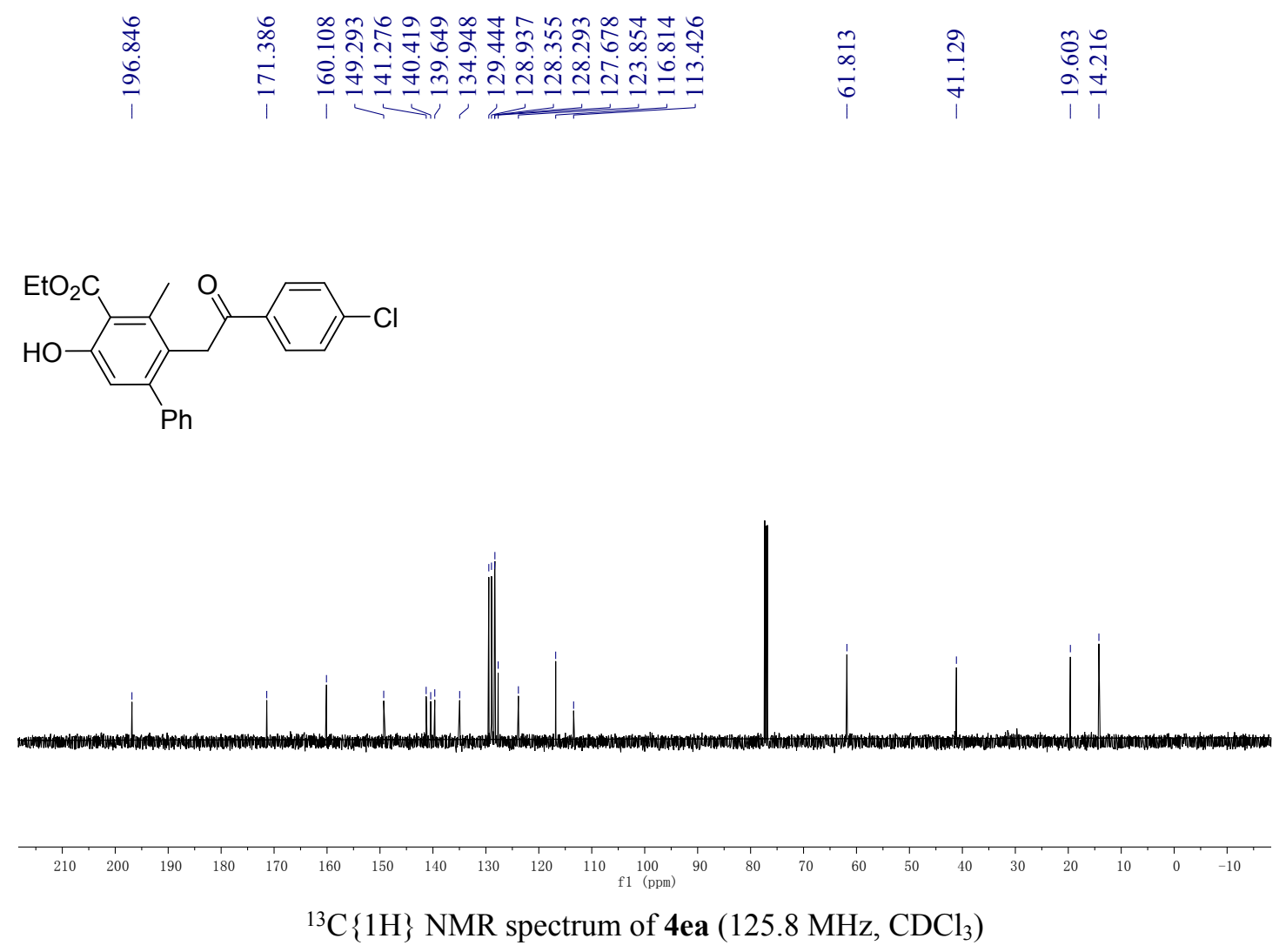

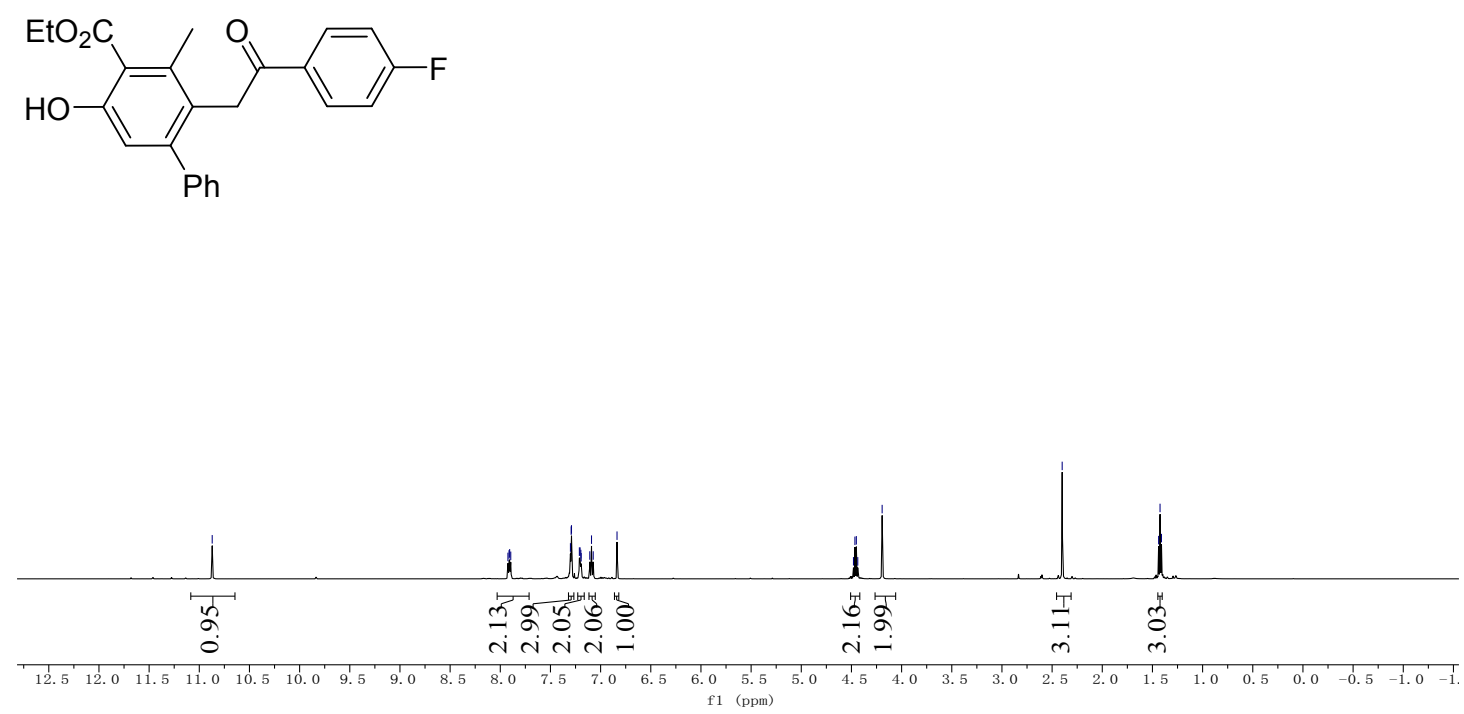

${ }^{1} \mathrm{H}$ NMR spectrum of $\mathbf{4 f a}\left(500 \mathrm{MHz}, \mathrm{CDCl}_{3}\right)$
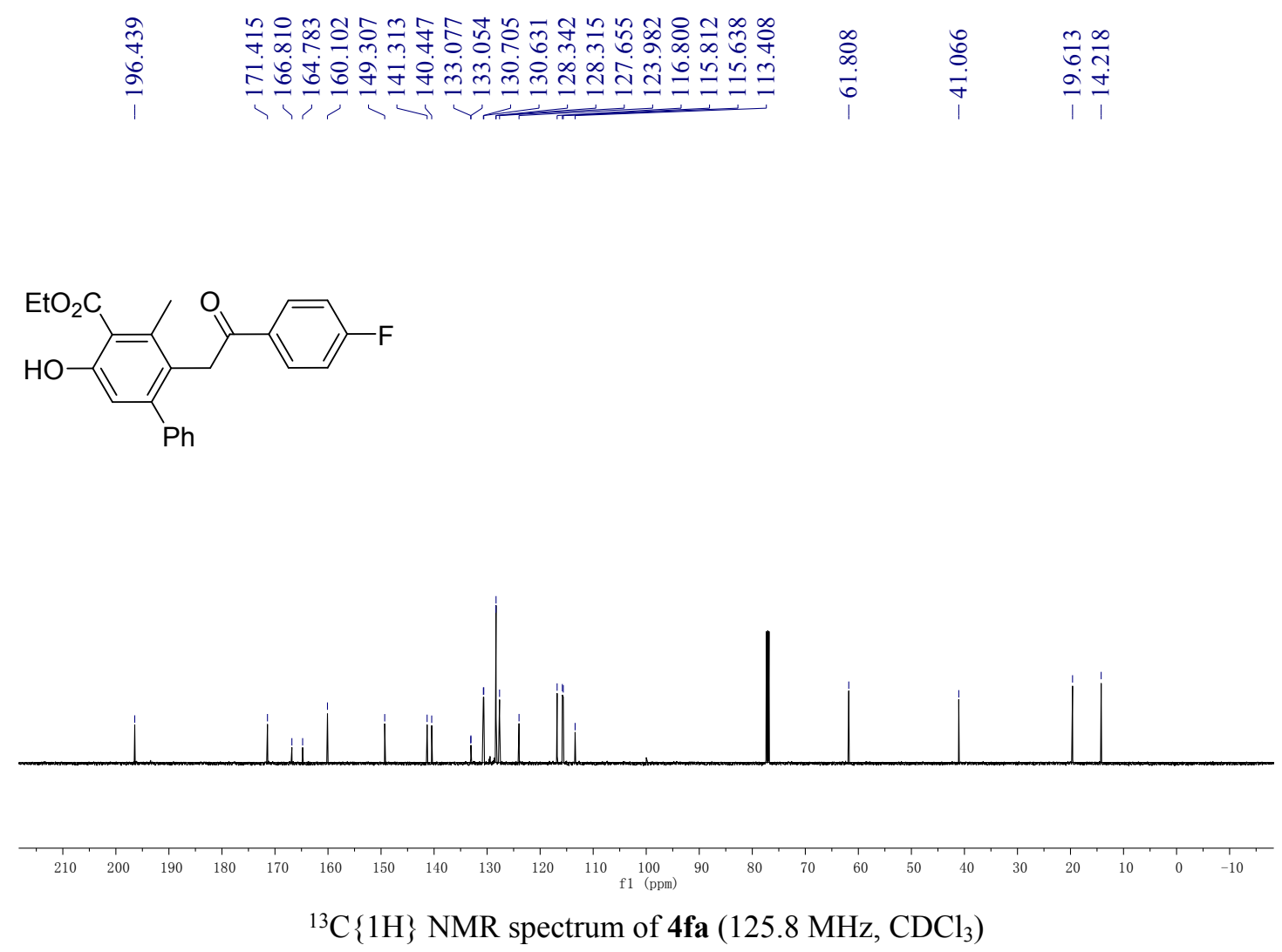
<smiles>C=[AsH2]</smiles>

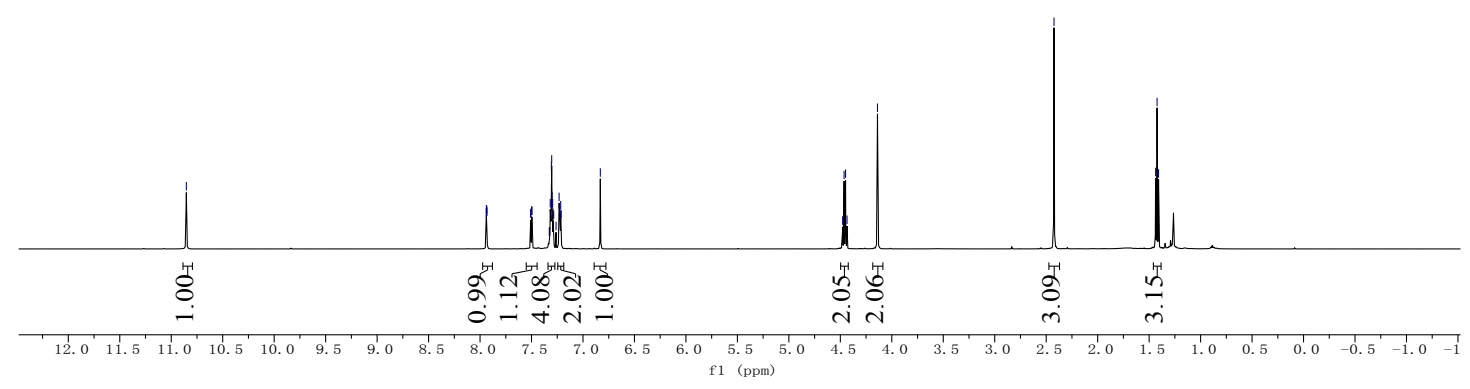

${ }^{1} \mathrm{H}$ NMR spectrum of $\mathbf{4 g a}\left(500 \mathrm{MHz}, \mathrm{CDCl}_{3}\right)$
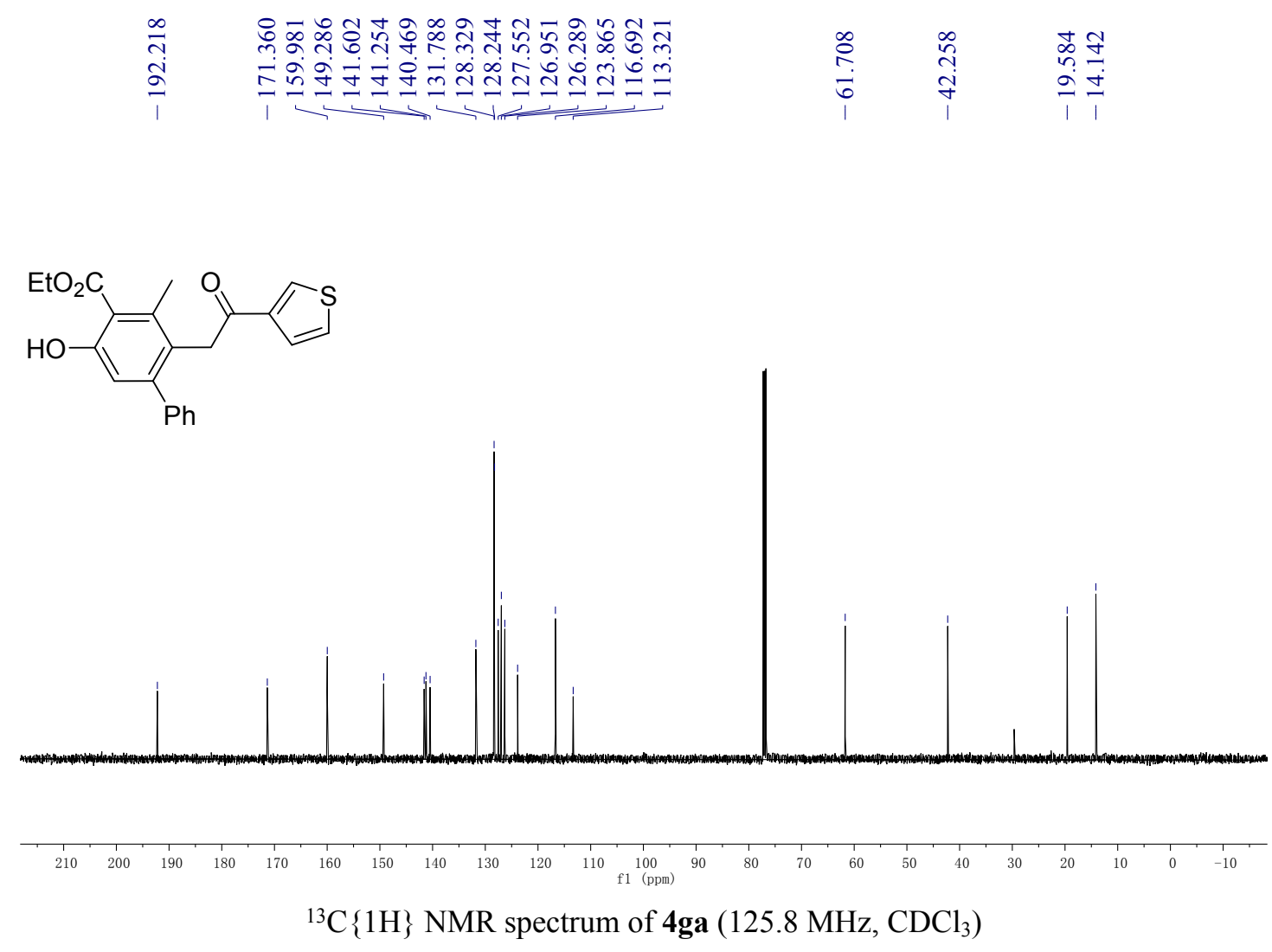


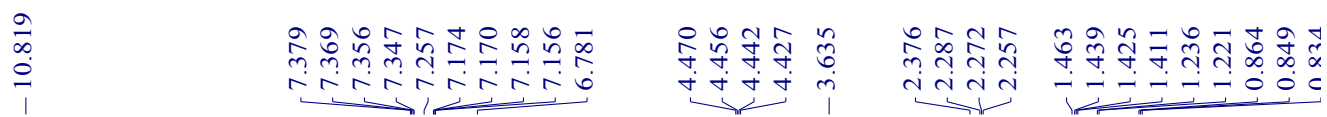
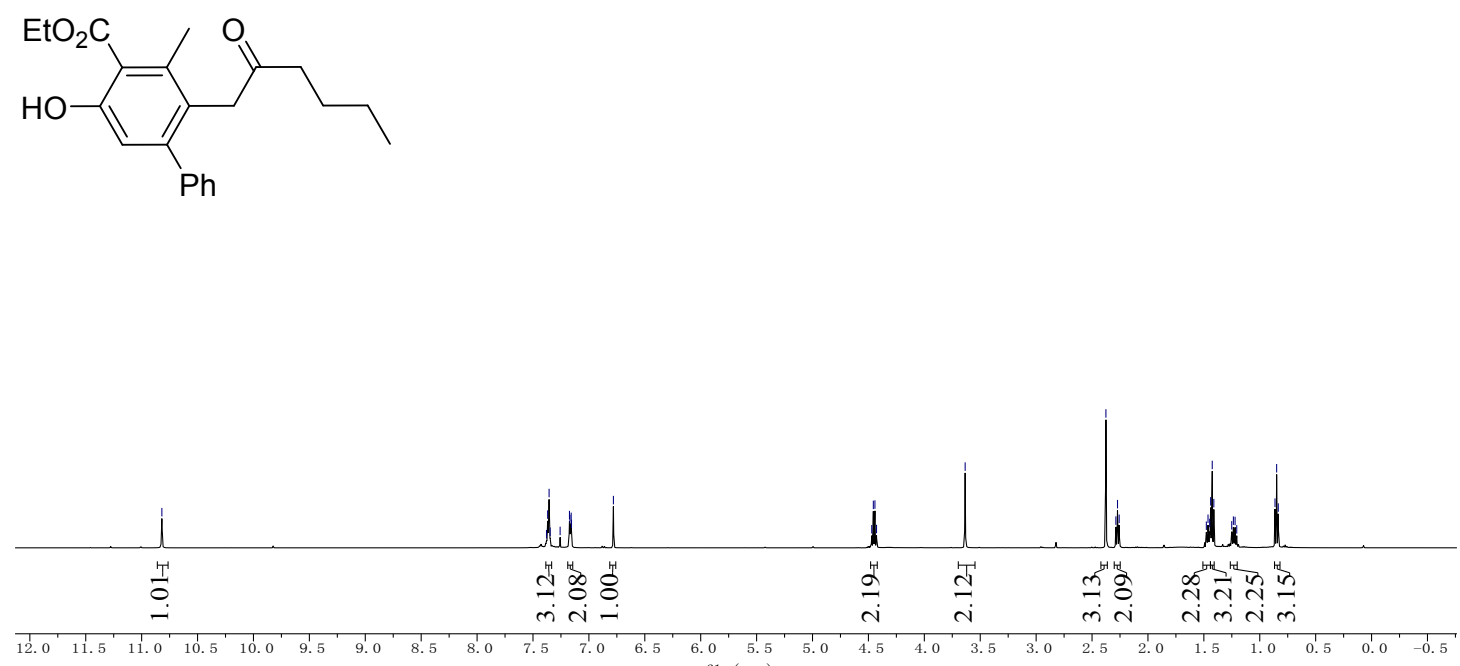

${ }^{1} \mathrm{H}$ NMR spectrum of 4 ha $\left(500 \mathrm{MHz}, \mathrm{CDCl}_{3}\right)$
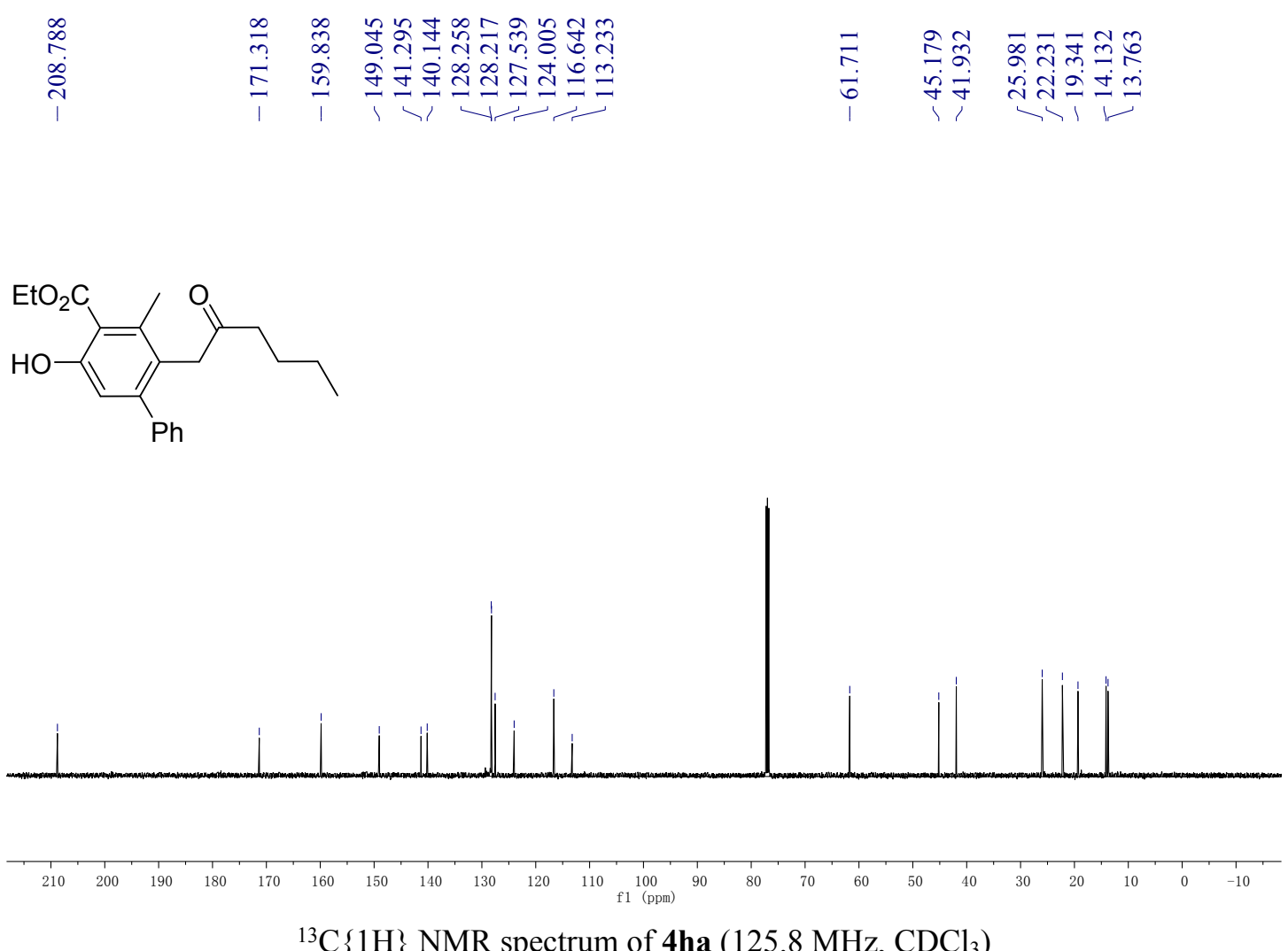

${ }^{13} \mathrm{C}\{1 \mathrm{H}\}$ NMR spectrum of tha $\left(125.8 \mathrm{MHz}, \mathrm{CDCl}_{3}\right)$ 

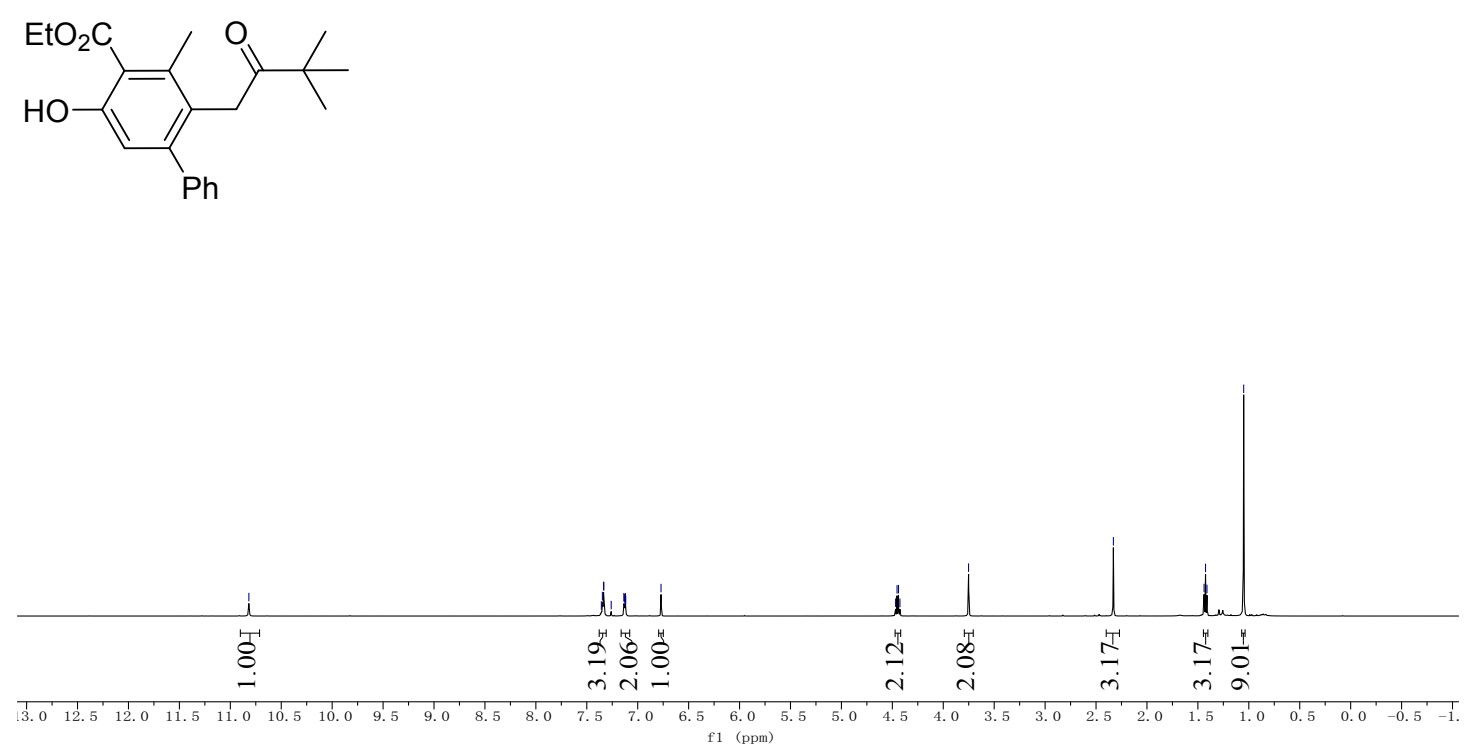

${ }^{1} \mathrm{H}$ NMR spectrum of 4 ia $\left(500 \mathrm{MHz}, \mathrm{CDCl}_{3}\right)$
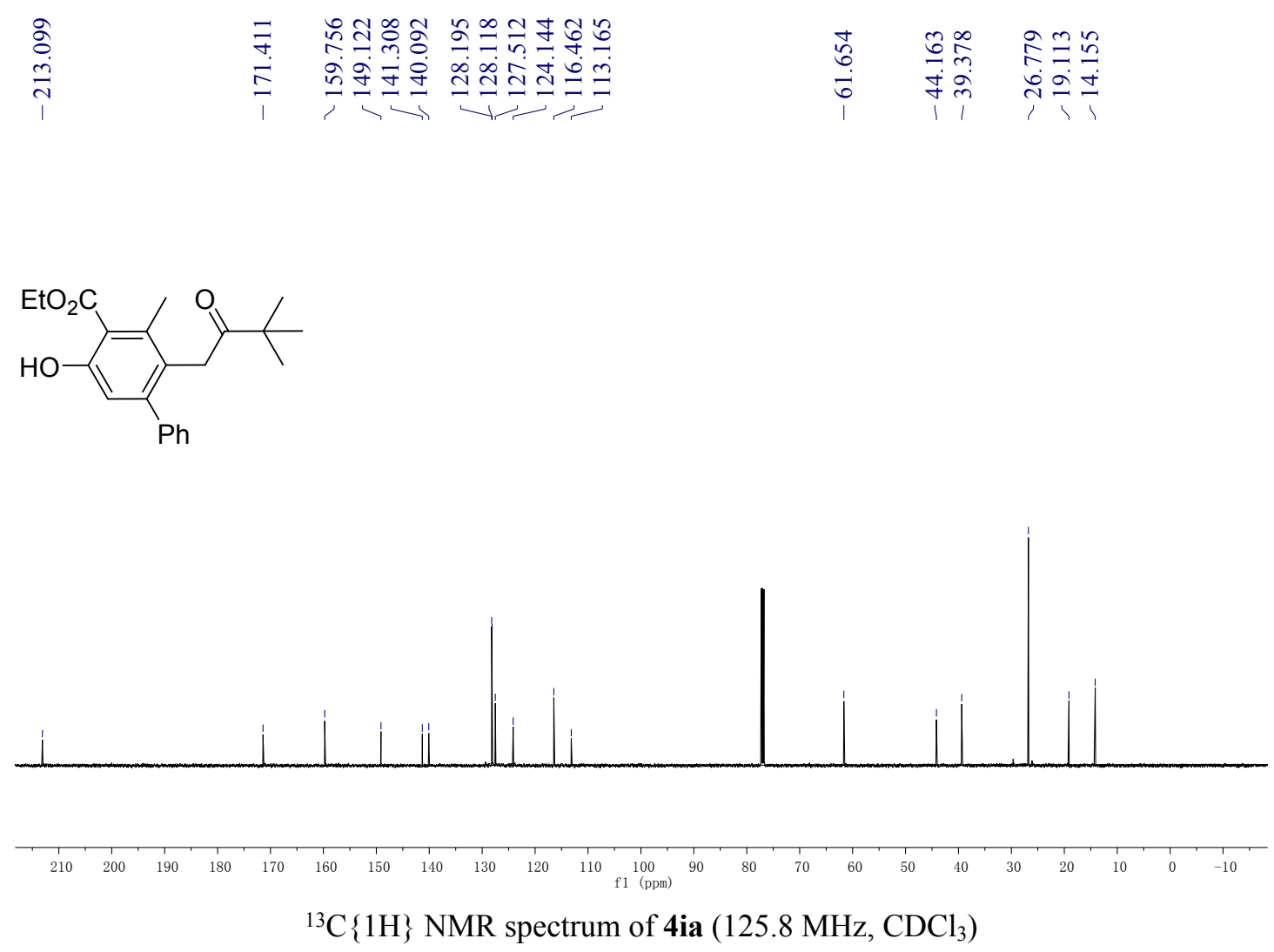
<smiles>Cc1c(CC(=O)c2ccccc2)cc(O)c(C(=O)c2ccccc2)c1-c1ccccc1</smiles>

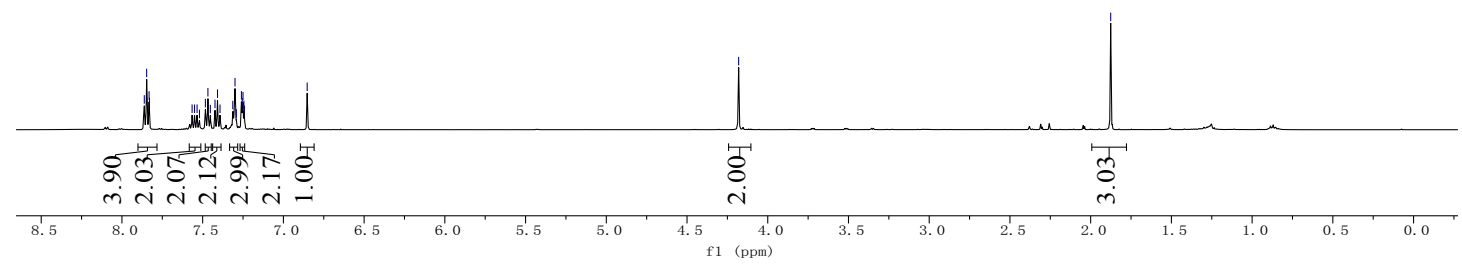

${ }^{1} \mathrm{H}$ NMR spectrum of $\mathbf{4 a b}\left(500 \mathrm{MHz}, \mathrm{CDCl}_{3}\right)$

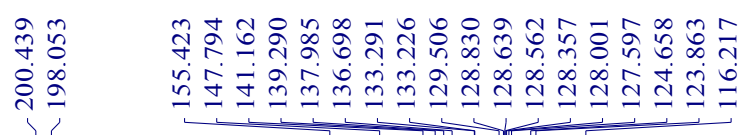

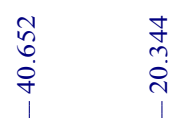<smiles>Cc1c(-c2ccccc2)cc(O)c(C(=O)c2ccccc2)c1CC(=O)c1ccccc1</smiles>
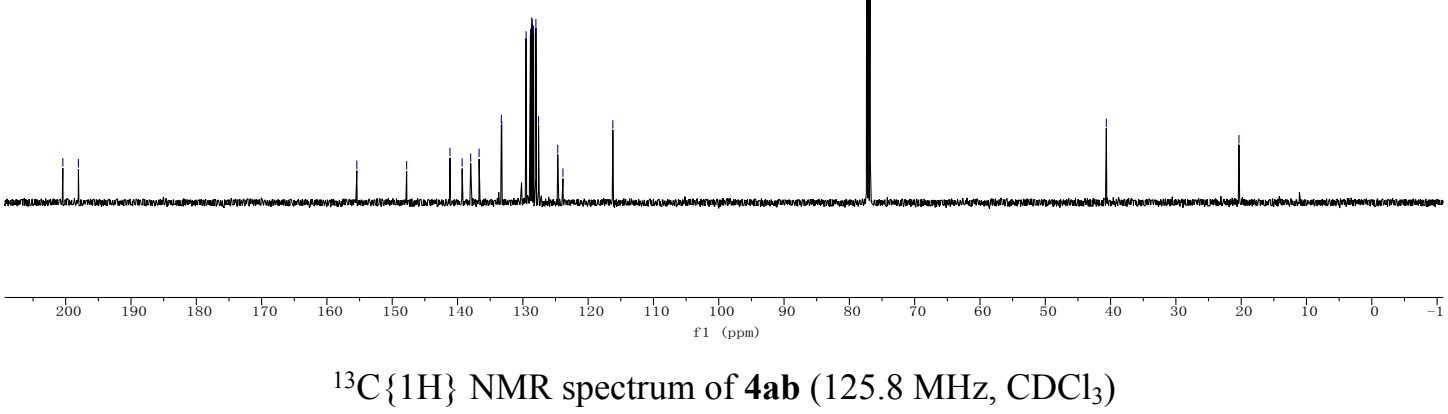

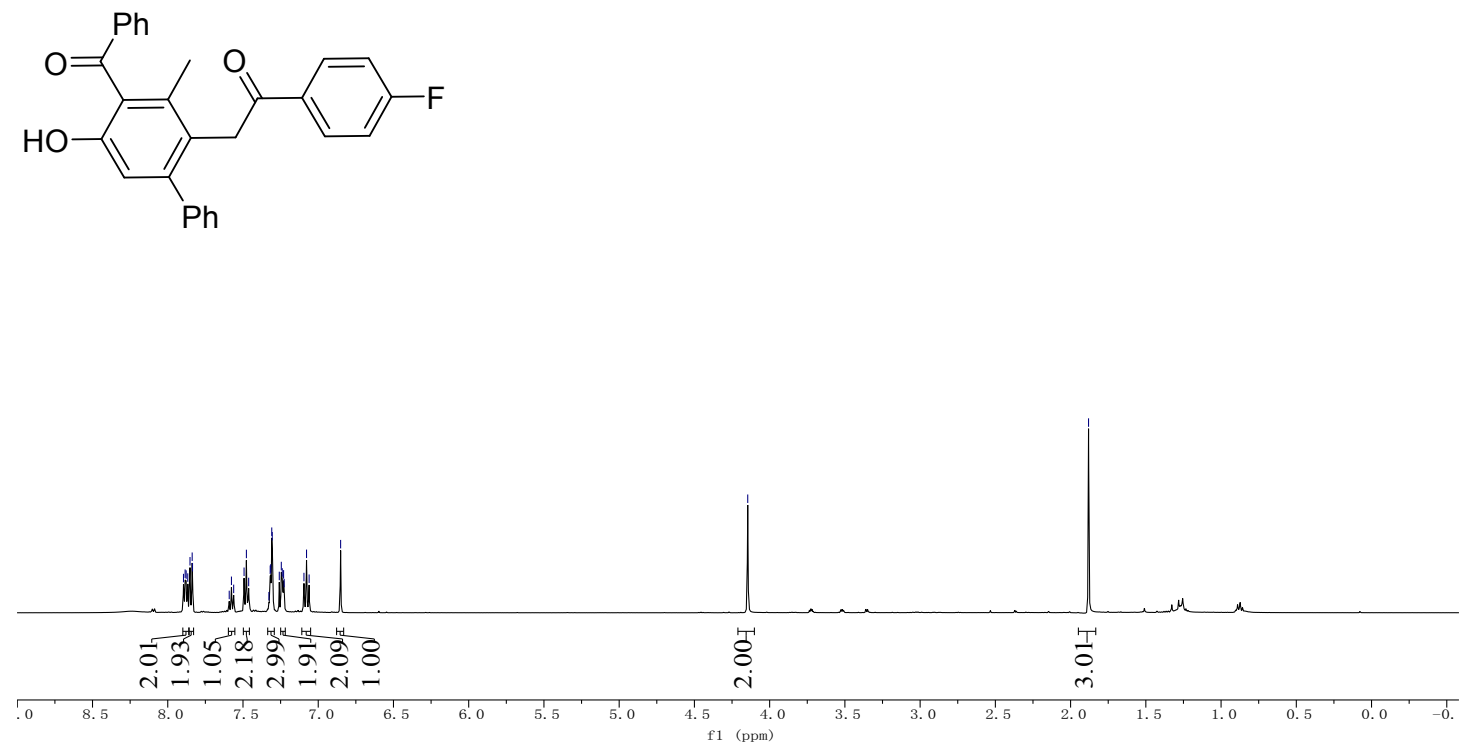

${ }^{1} \mathrm{H}$ NMR spectrum of $\mathbf{4 c b}\left(500 \mathrm{MHz}, \mathrm{CDCl}_{3}\right)$

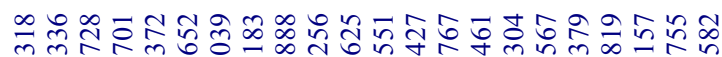

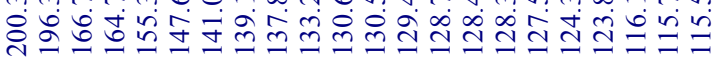

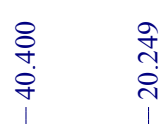
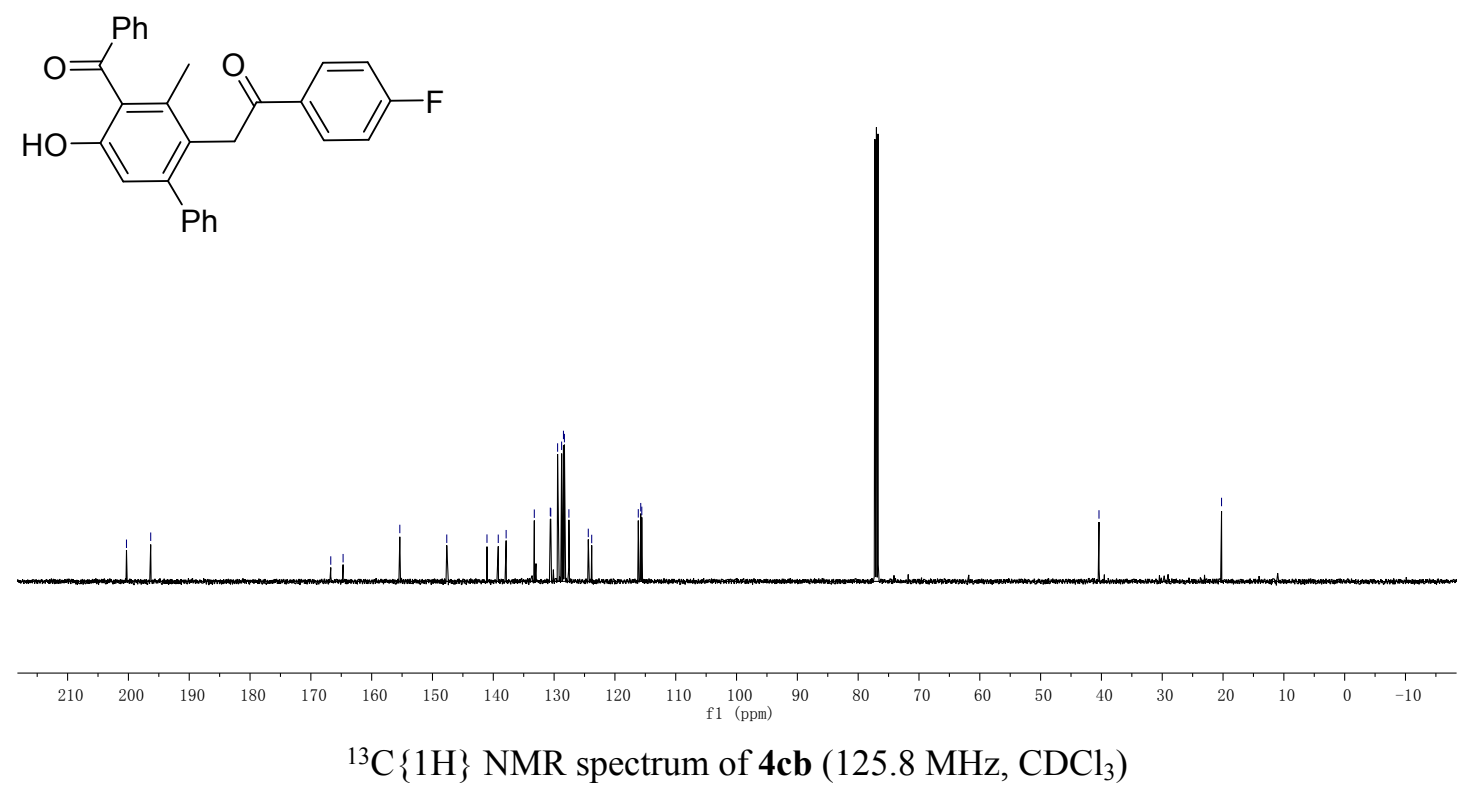
<smiles>CCC(=O)Cc1c(-c2ccccc2)cc(O)c(C(=O)c2ccccc2)c1C</smiles>

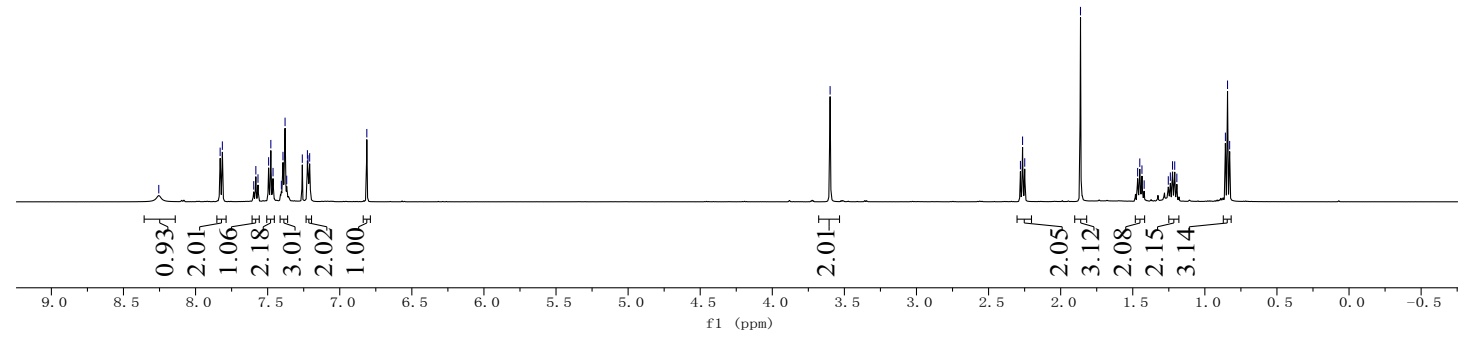

${ }^{1} \mathrm{H}$ NMR spectrum of $4 \mathbf{e b}\left(500 \mathrm{MHz}, \mathrm{CDCl}_{3}\right)$

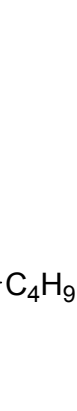

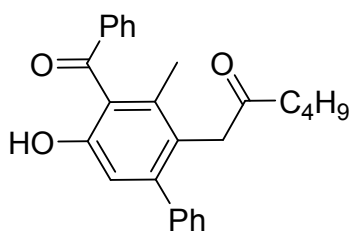
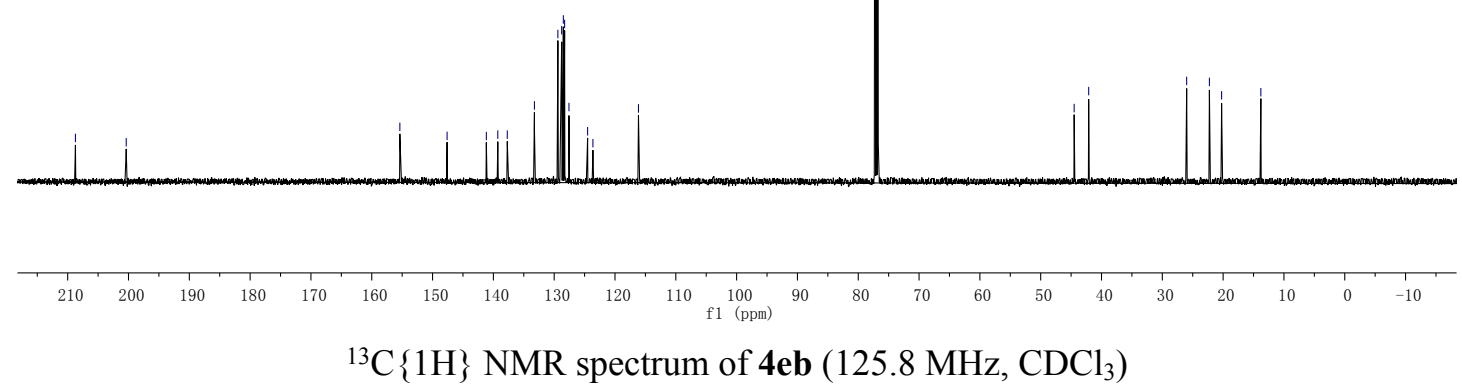
<smiles>CCOC(=O)C(=N)C(=O)CC(C(C)=O)C(=Cc1ccccc1)c1ccccc1</smiles>

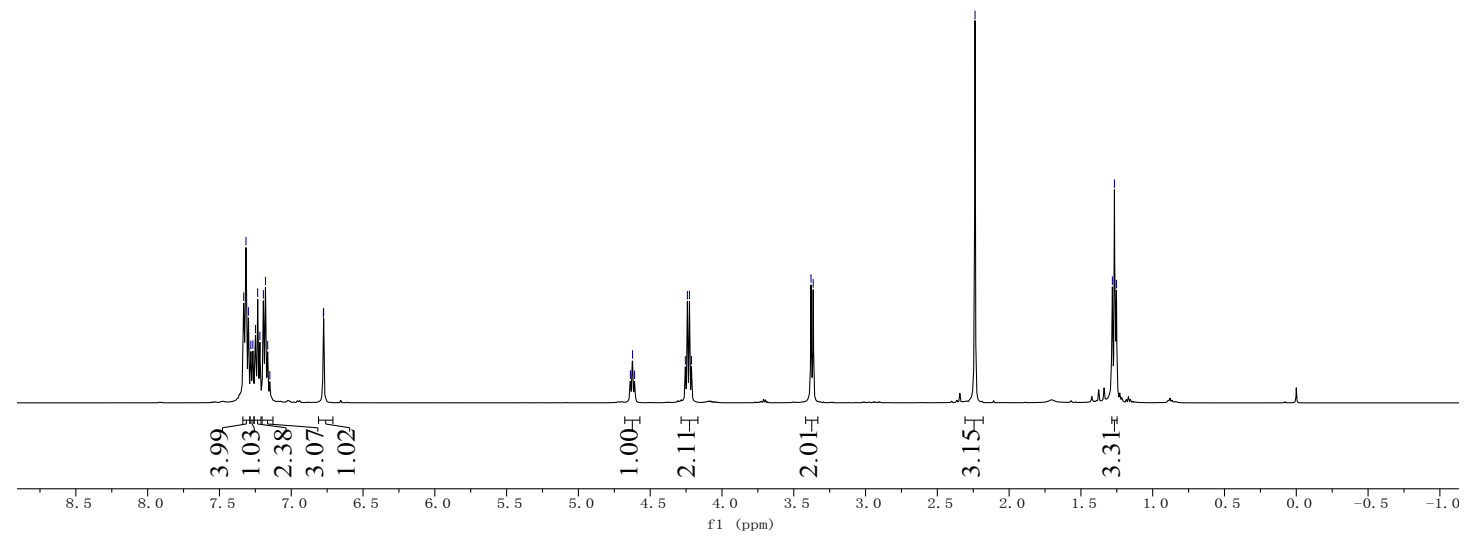

${ }^{1} \mathrm{H}$ NMR spectrum of $\mathbf{1 a a}\left(500 \mathrm{MHz}, \mathrm{CDCl}_{3}\right.$

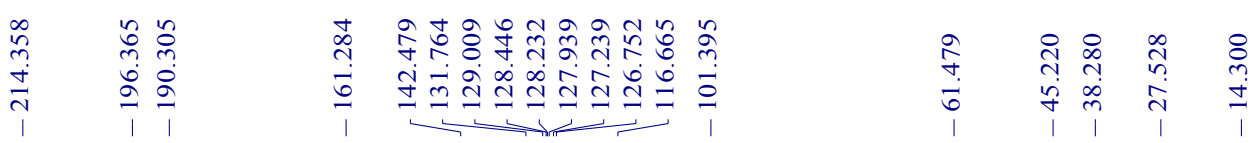<smiles>CCOC(=O)C(=N)C(=O)CC(C(C)=O)C(=Cc1ccccc1)c1ccccc1</smiles>
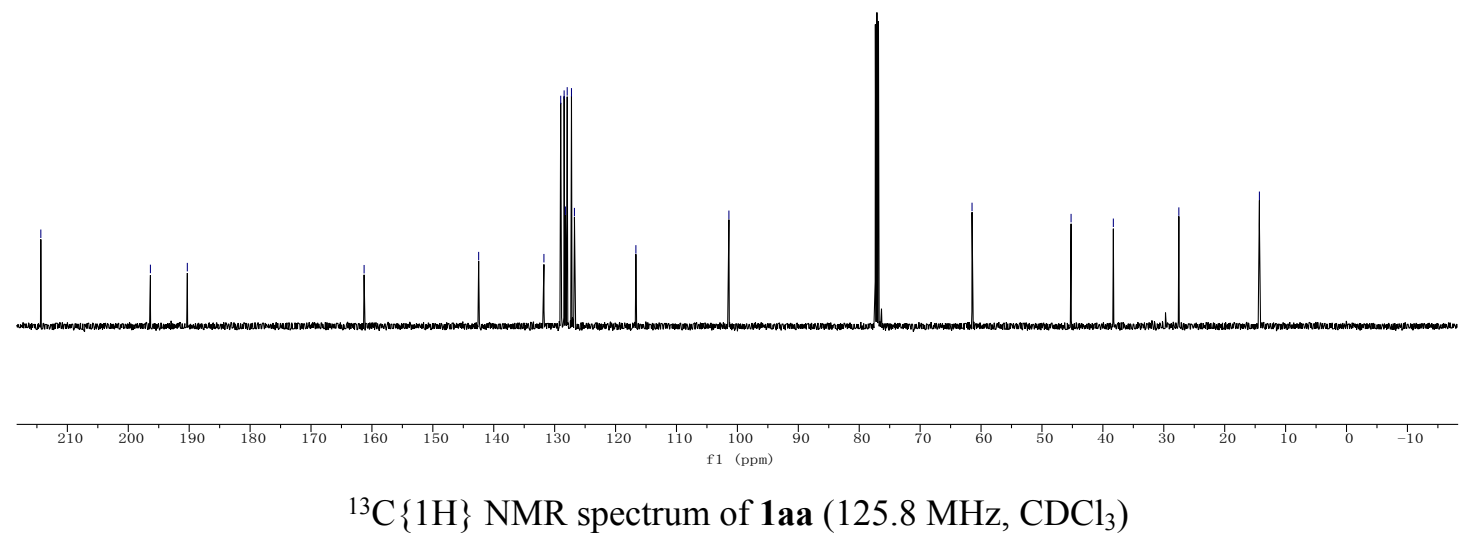


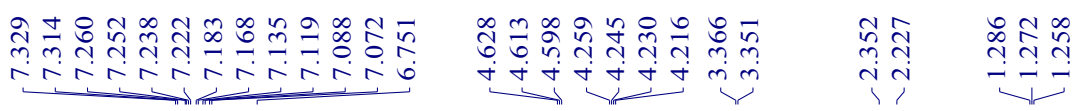<smiles>CCOC(=O)C(=O)C(=O)CC(C(C)=O)C(=C=CCc1ccc(C)cc1)c1ccccc1</smiles>

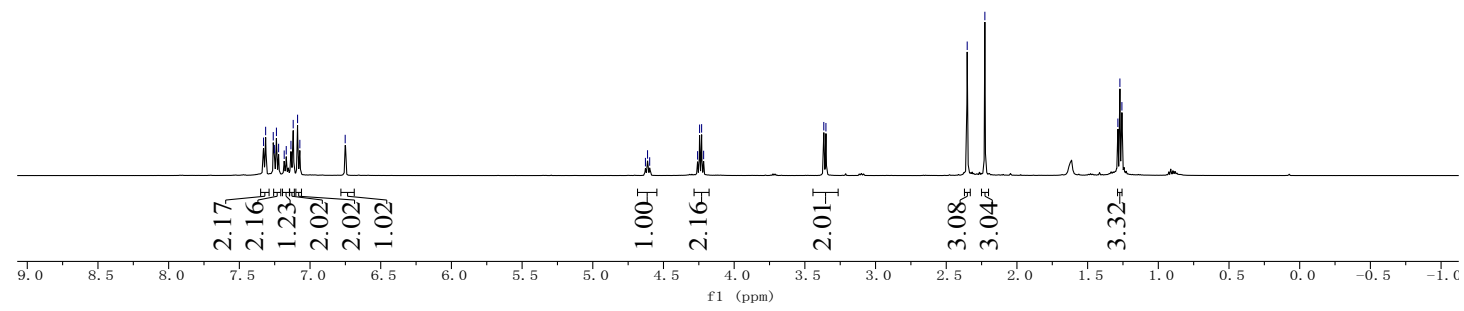

${ }^{1} \mathrm{H}$ NMR spectrum of $\mathbf{1 b a}\left(500 \mathrm{MHz}, \mathrm{CDCl}_{3}\right)$

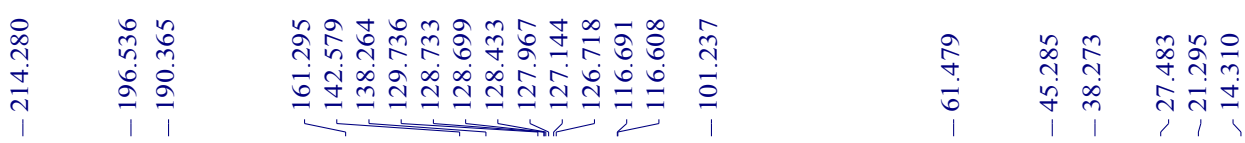<smiles>CCOC(=O)C(=[N+]=O)C(=O)CC(C(C)=O)C(=C=CCc1ccc(C)cc1)c1ccccc1</smiles>
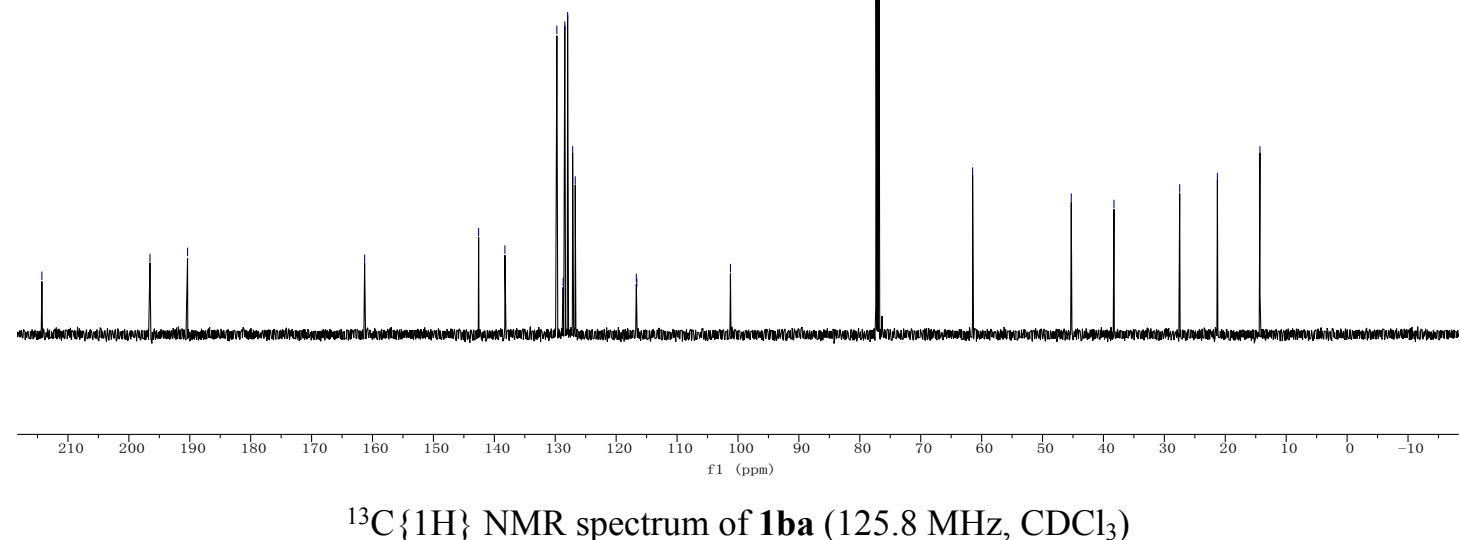

${ }^{3} \mathrm{C}\{1 \mathrm{H}\}$ NMR spectrum of $\mathbf{1 b a}\left(125.8 \mathrm{MHz}, \mathrm{CDCl}_{3}\right)$ 
<smiles>CCOC(=O)C(=N)C(=O)CC(C(C)=O)C(=Cc1ccc(OC)cc1)c1ccccc1</smiles>

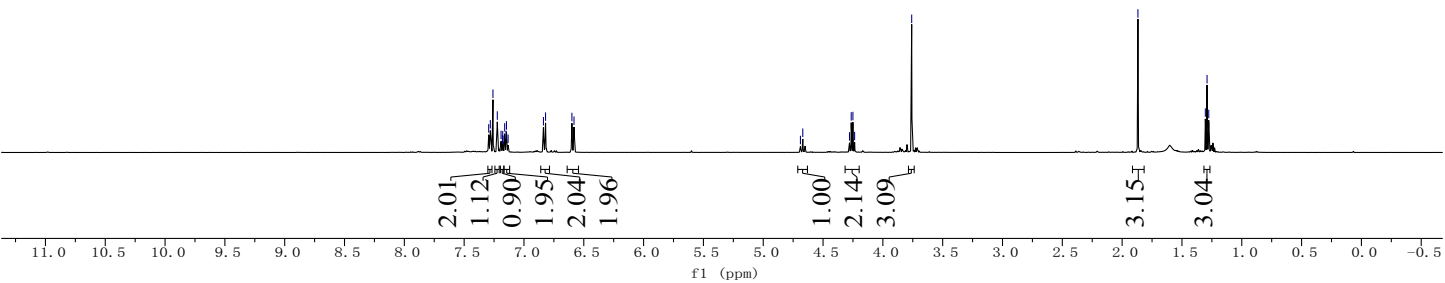

${ }^{1} \mathrm{H}$ NMR spectrum of $\mathbf{1 c a}\left(500 \mathrm{MHz}, \mathrm{CDCl}_{3}\right)$

\begin{tabular}{|c|c|c|c|c|c|c|}
\hline $\begin{array}{l}\dot{0} \\
\dot{\sigma} \\
\text { na }\end{array}$ & 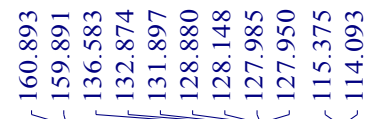 & $\begin{array}{l}\vec{\gamma} \\
\infty \\
\infty\end{array}$ & & $\infty$ & & \\
\hline
\end{tabular}<smiles>CCOC(=O)C(=N)C(=O)CC(C(C)=O)C(=Cc1ccc(OC)cc1)c1ccccc1</smiles>
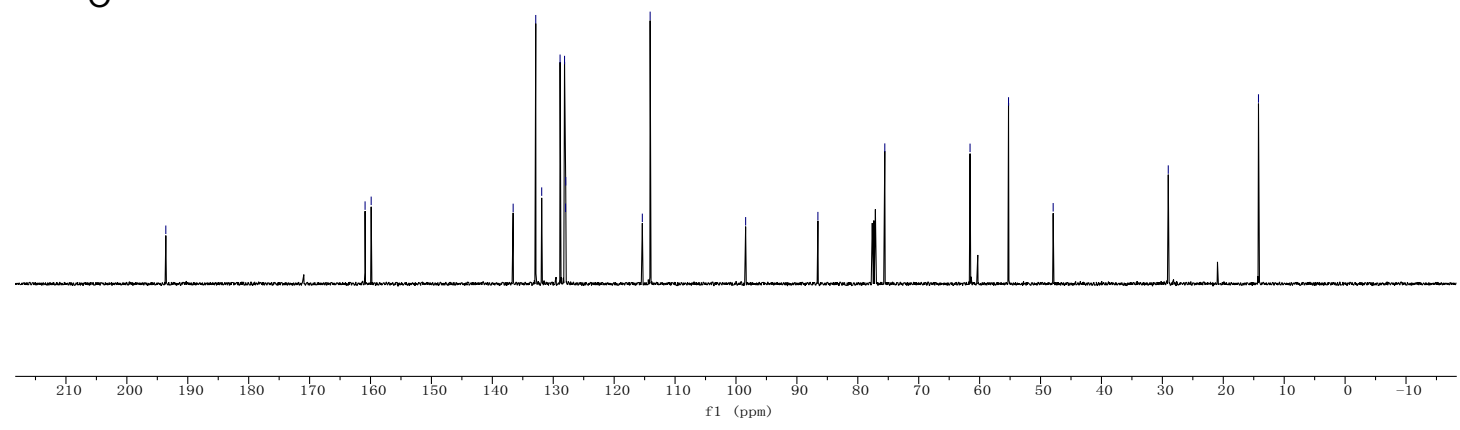

${ }^{13} \mathrm{C}\{1 \mathrm{H}\} \mathrm{NMR}$ spectrum of $\mathbf{1 c a}\left(125.8 \mathrm{MHz}, \mathrm{CDCl}_{3}\right)$ 
<smiles>CCOC(=O)C(=[W])C(=O)CC(C(C)=O)C(=Cc1ccc(Br)cc1)c1ccccc1</smiles>

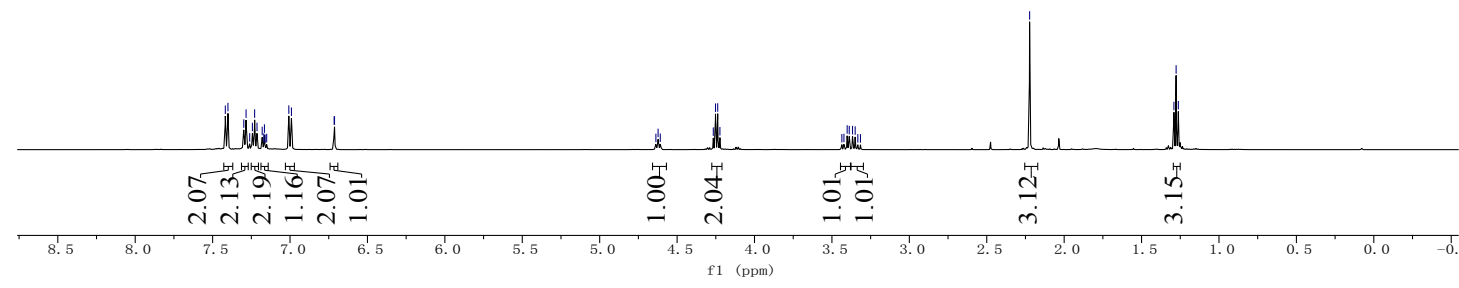

${ }^{1} \mathrm{H}$ NMR spectrum of $\mathbf{1 d a}\left(500 \mathrm{MHz}, \mathrm{CDCl}_{3}\right)$
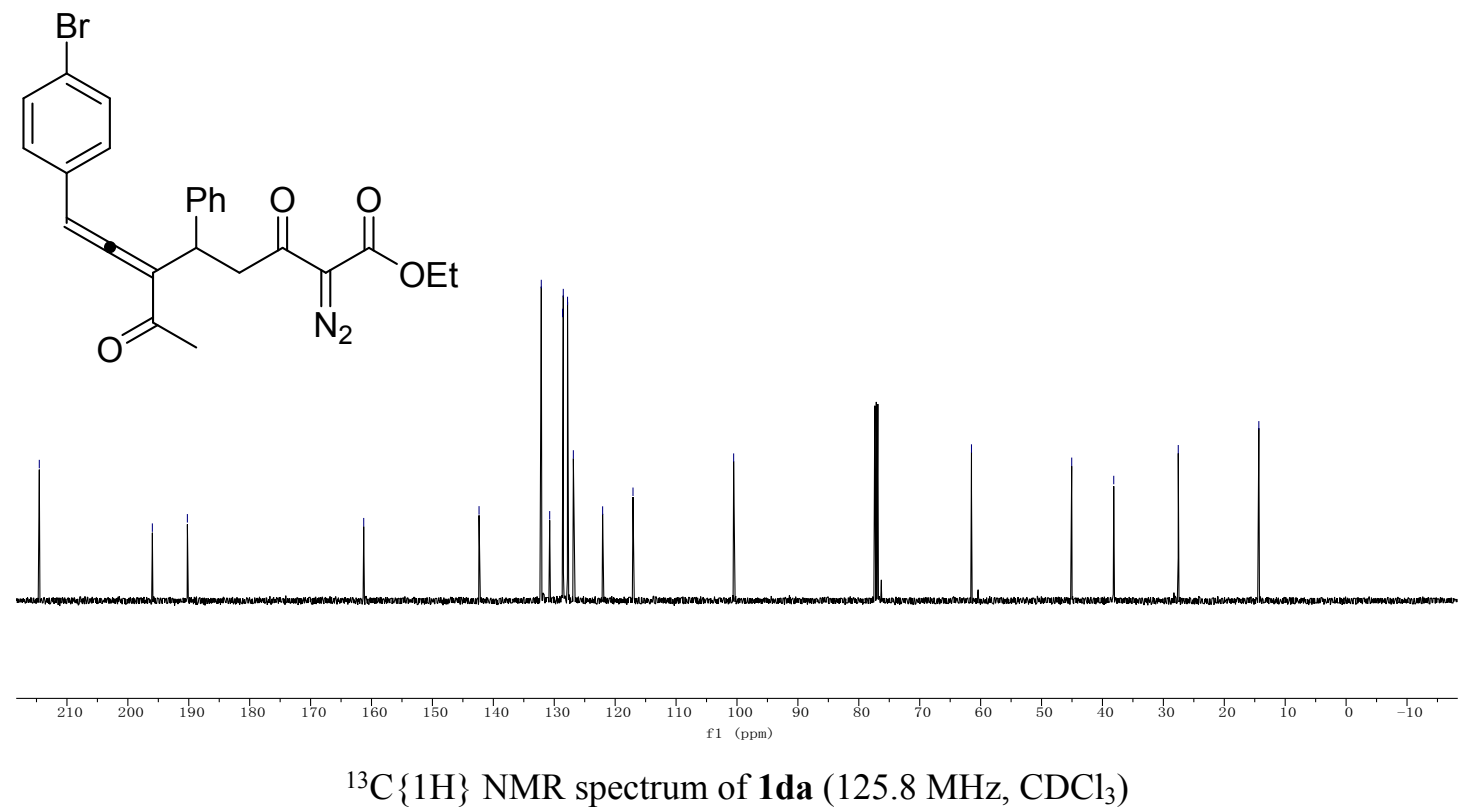
<smiles>CCOC(=O)C(=N)C(=O)CC(C(C)=O)C(=Cc1ccc(Cl)cc1)c1ccccc1</smiles>

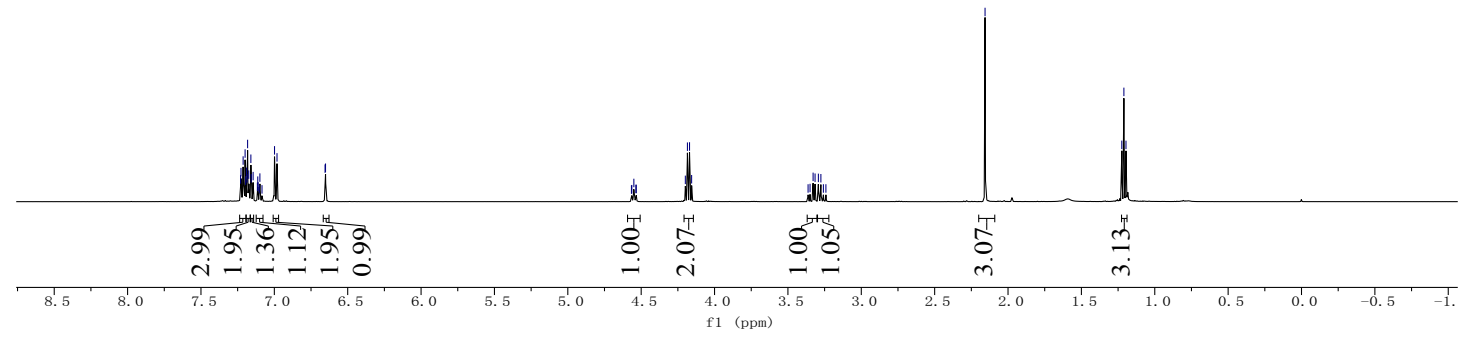

${ }^{1} \mathrm{H}$ NMR spectrum of $\mathbf{1 e a}\left(500 \mathrm{MHz}, \mathrm{CDCl}_{3}\right)$

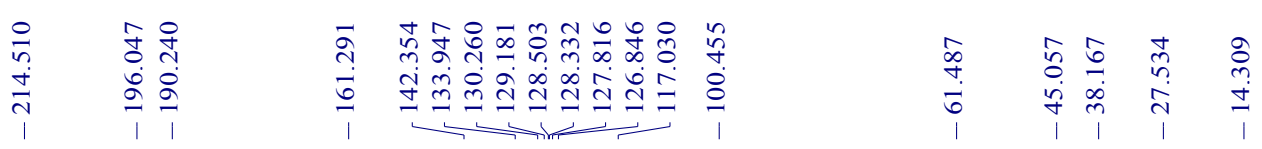
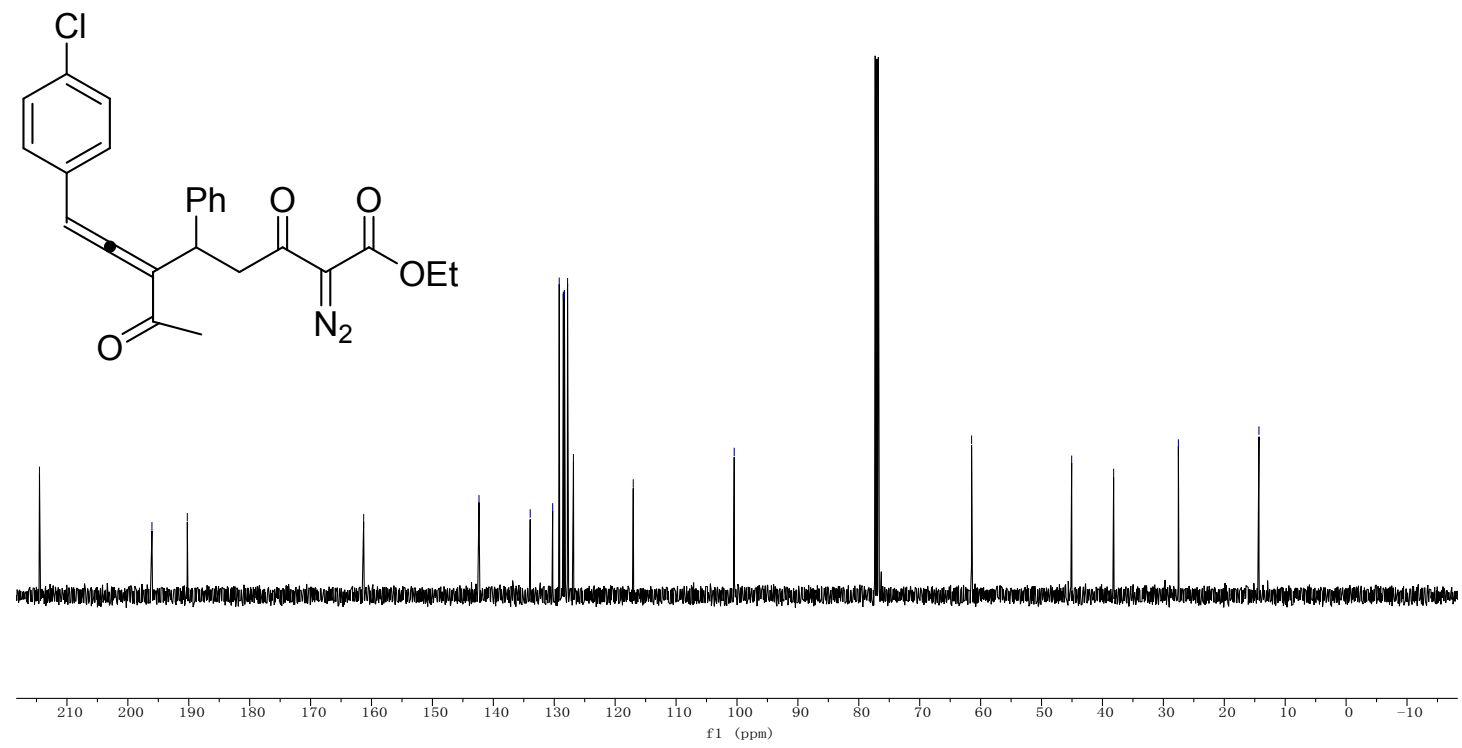

${ }^{13} \mathrm{C}\{1 \mathrm{H}\} \mathrm{NMR}$ spectrum of 1 ea $\left(125.8 \mathrm{MHz}, \mathrm{CDCl}_{3}\right)$ 
<smiles>CCOC(=O)C(=N)C(=O)CC(C(C)=O)C(=Cc1ccc(F)cc1)c1ccccc1</smiles>

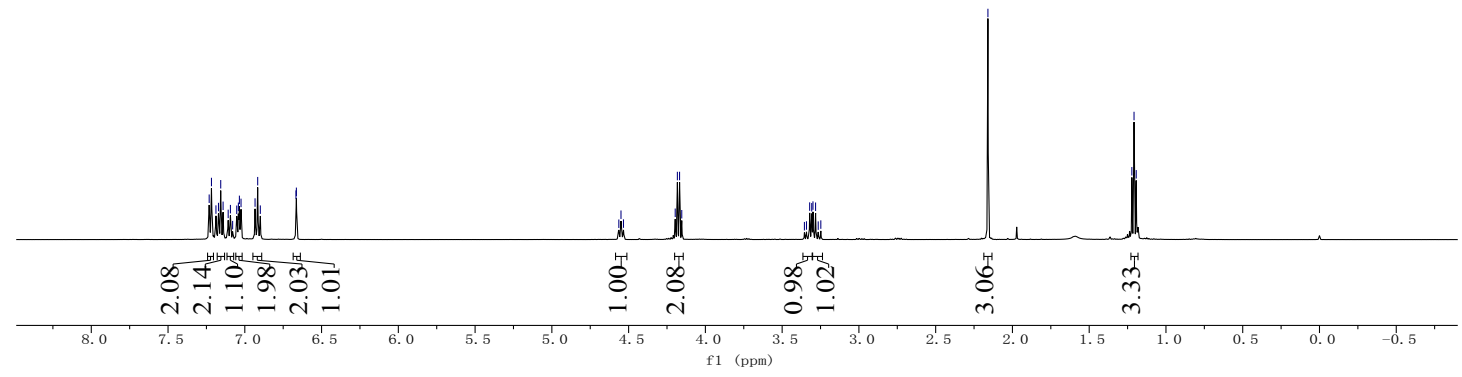

${ }^{1} \mathrm{H}$ NMR spectrum of $\mathbf{1 f a}\left(500 \mathrm{MHz}, \mathrm{CDCl}_{3}\right)$

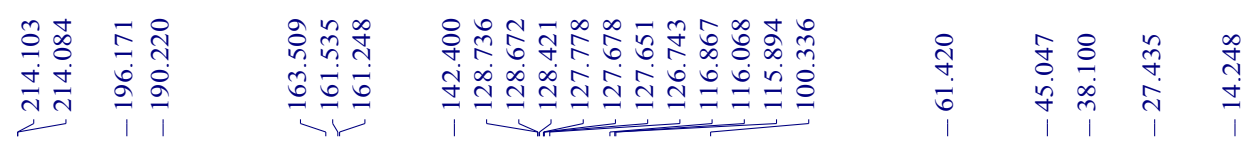<smiles>CCOC(=O)C(=N)C(=O)CC(C(C)=O)C(=Cc1ccc(F)cc1)c1ccccc1</smiles>

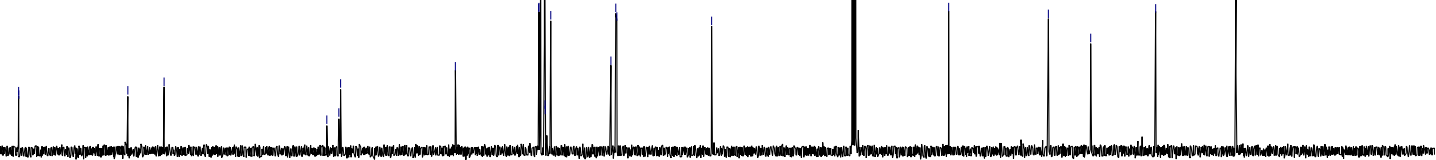

${ }^{13} \mathrm{C}\{1 \mathrm{H}\}$ NMR spectrum of $\mathbf{1 f a}\left(125.8 \mathrm{MHz}, \mathrm{CDCl}_{3}\right)$ 


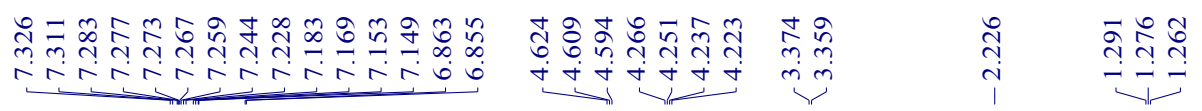<smiles>CCOC(=O)C(=[W])C(=O)CC(C(C)=O)C(=C=Cc1ccsc1)c1ccccc1</smiles>

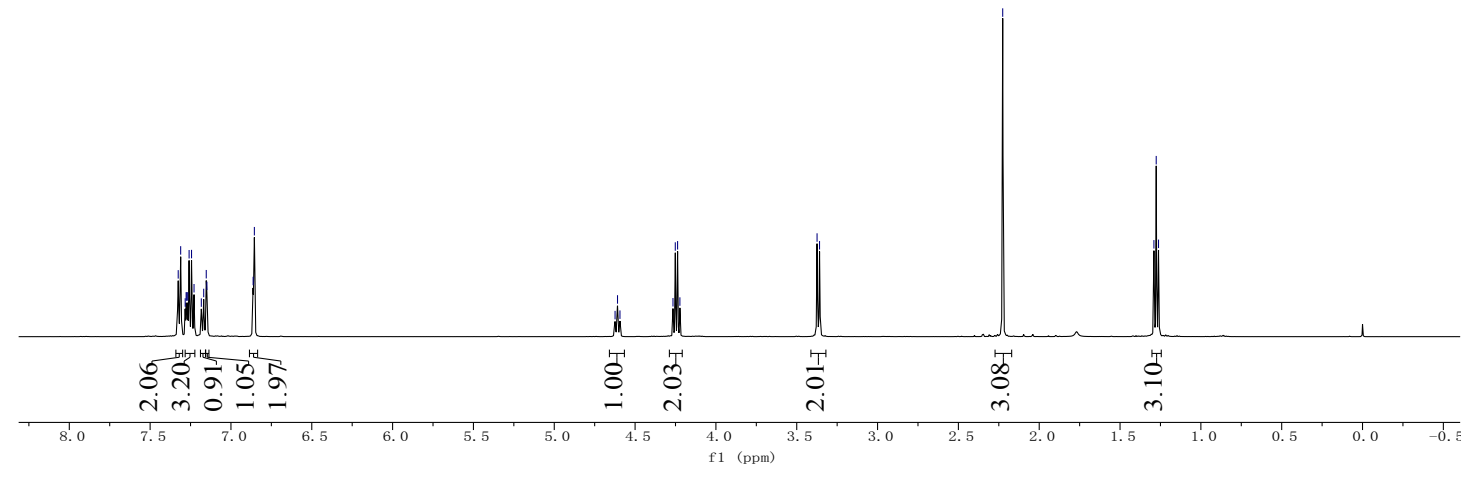

${ }^{1} \mathrm{H}$ NMR spectrum of 1 ga $\left(500 \mathrm{MHz}, \mathrm{CDCl}_{3}\right)$

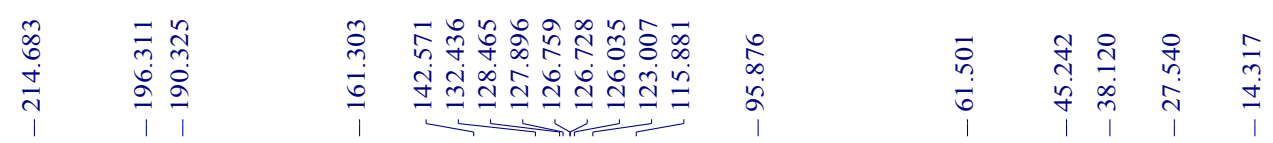<smiles>CCOC(=O)C(=[W])C(=O)CC(C(C)=O)C(=Cc1ccsc1)c1ccccc1</smiles>
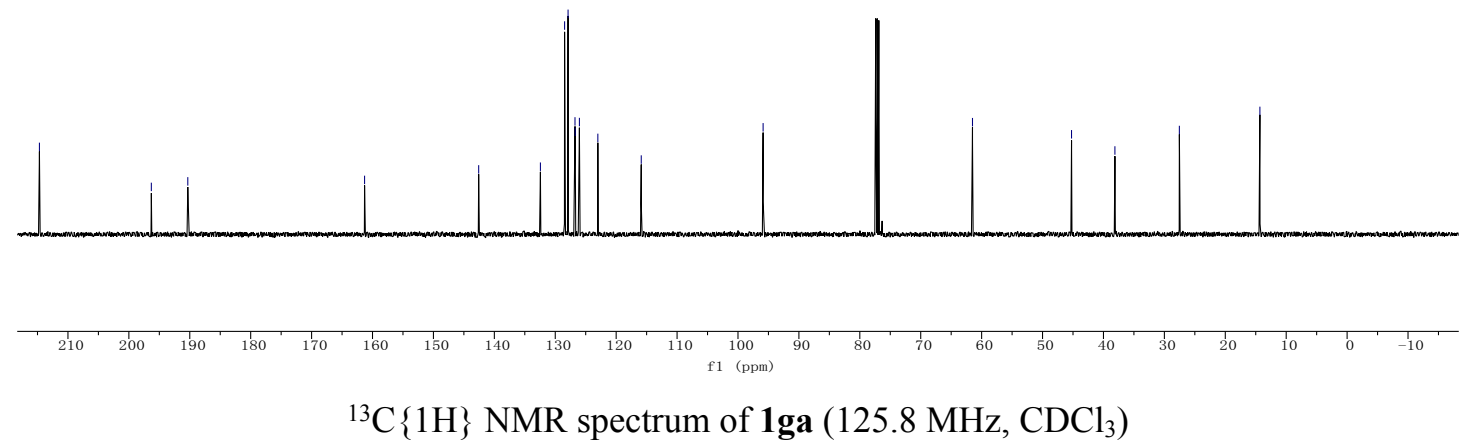

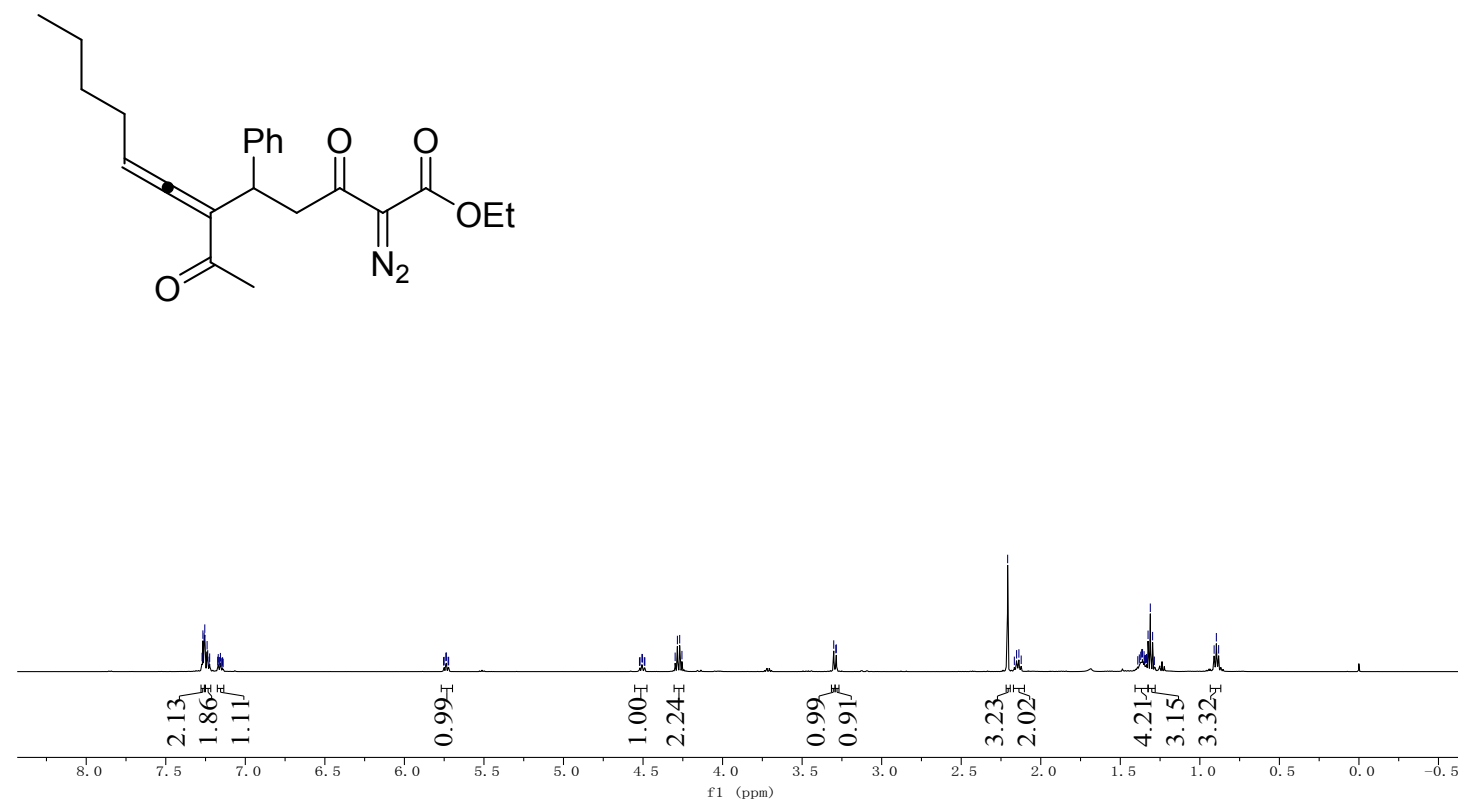

${ }^{1} \mathrm{H}$ NMR spectrum of $\mathbf{1 h a}\left(500 \mathrm{MHz}, \mathrm{CDCl}_{3}\right)$

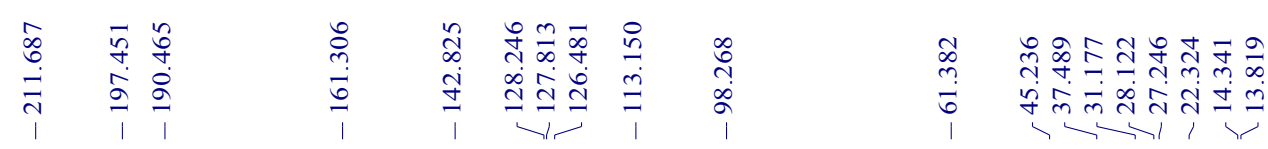<smiles>CCCCC=C=C(C(C)=O)C(CC(=O)C(=N)OCC)c1ccccc1</smiles>
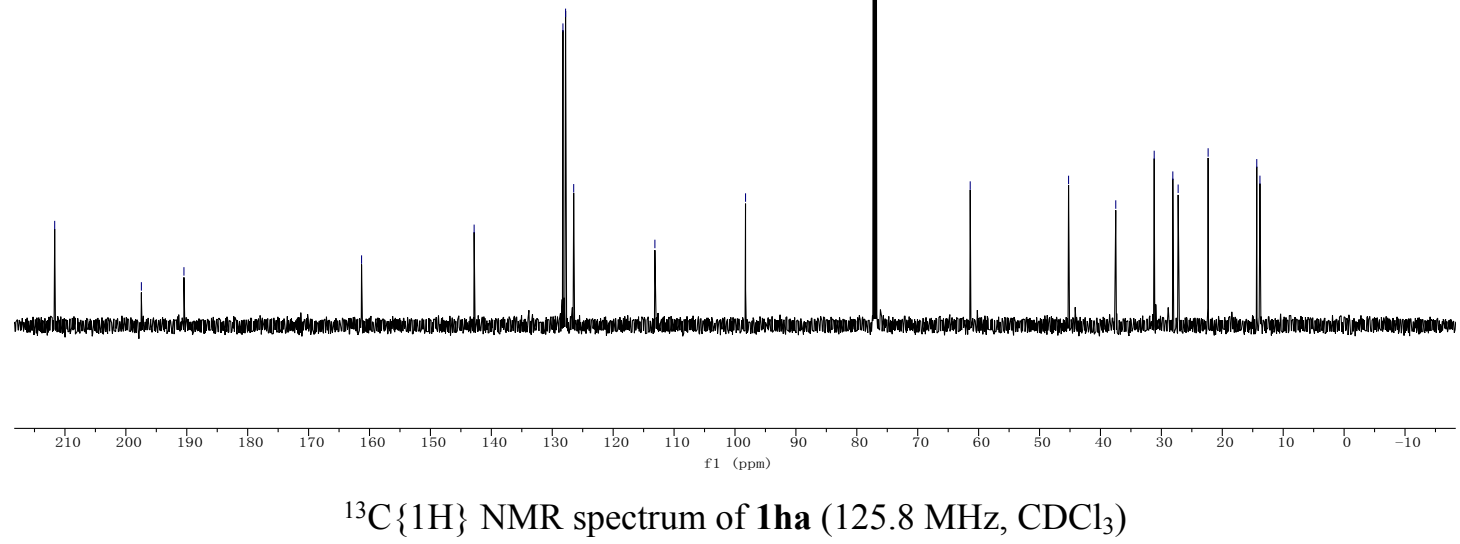
<smiles>CCOC(=O)C(=N)C(=O)CC(C(C)=O)C(=CC(C)(C)C)c1ccccc1</smiles>

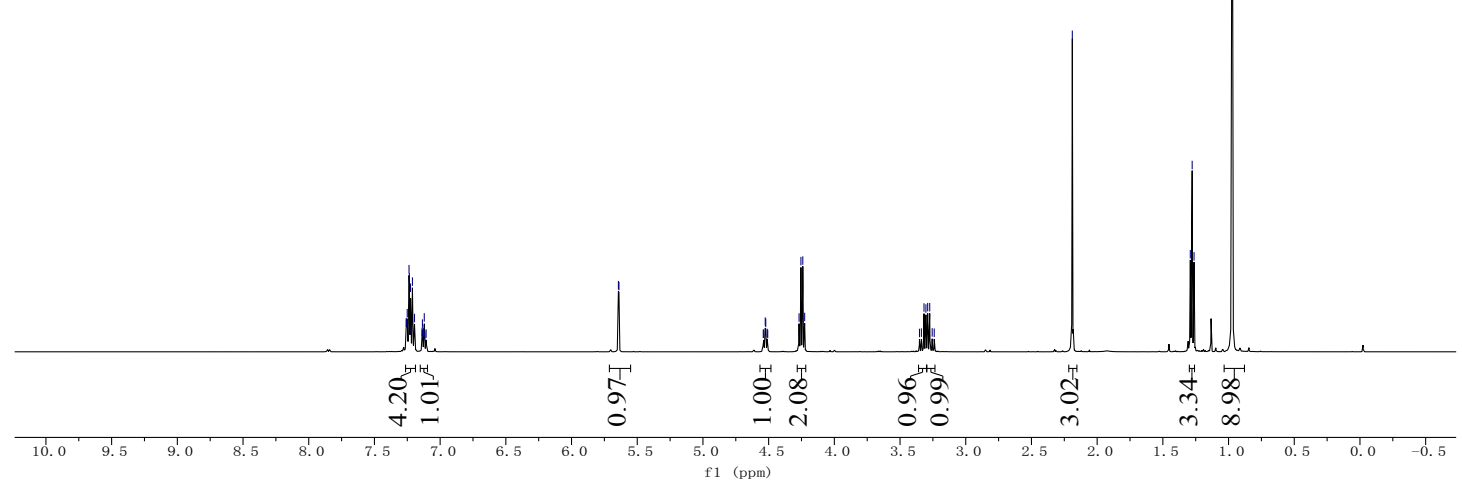

${ }^{1} \mathrm{H}$ NMR spectrum of $\mathbf{1} \mathbf{i a}\left(500 \mathrm{MHz}, \mathrm{CDCl}_{3}\right)$
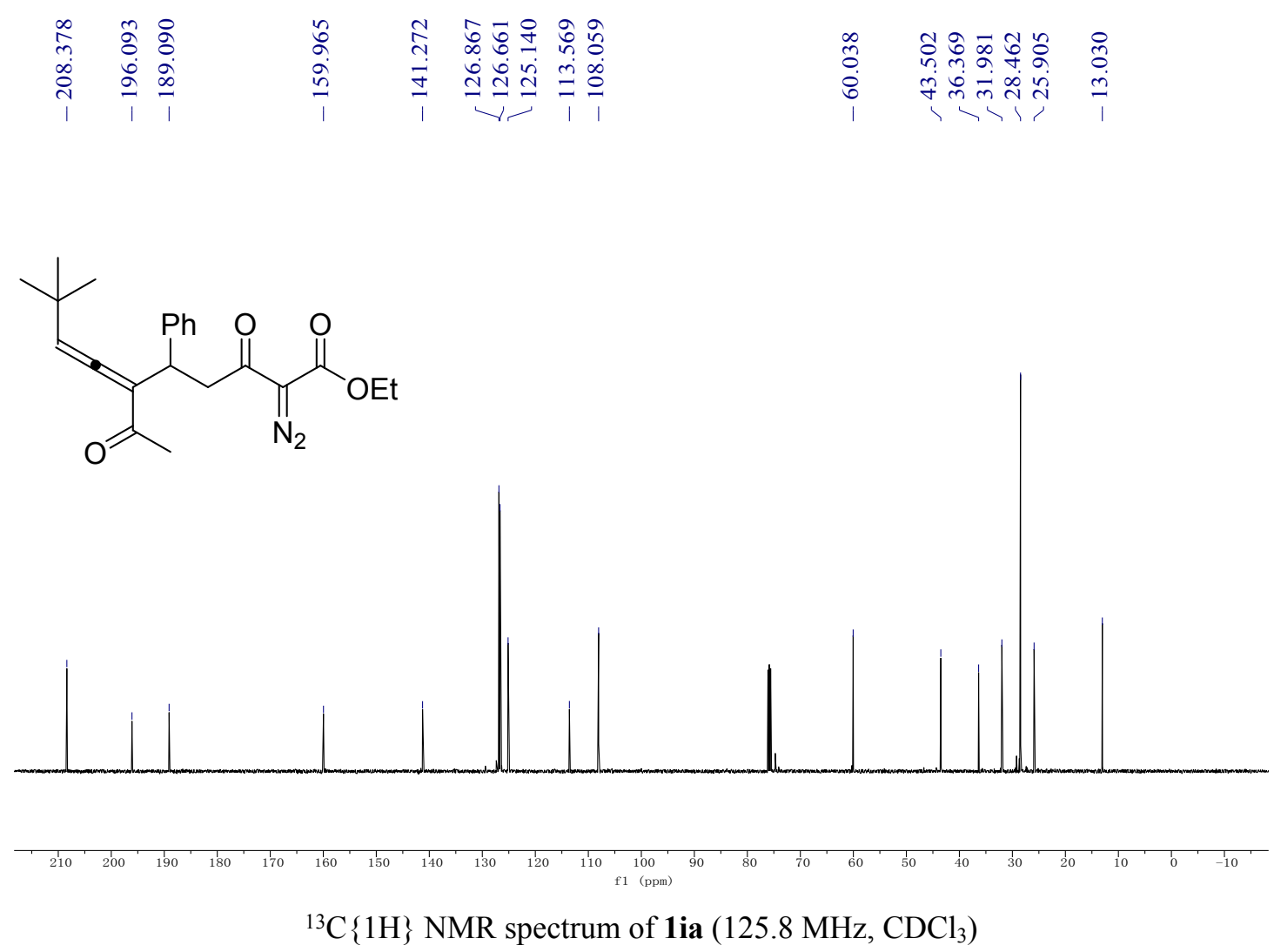
<smiles>C=C=C(C(C)=O)C(CC(=O)C(=N)C(=O)OCC)c1ccccc1</smiles>

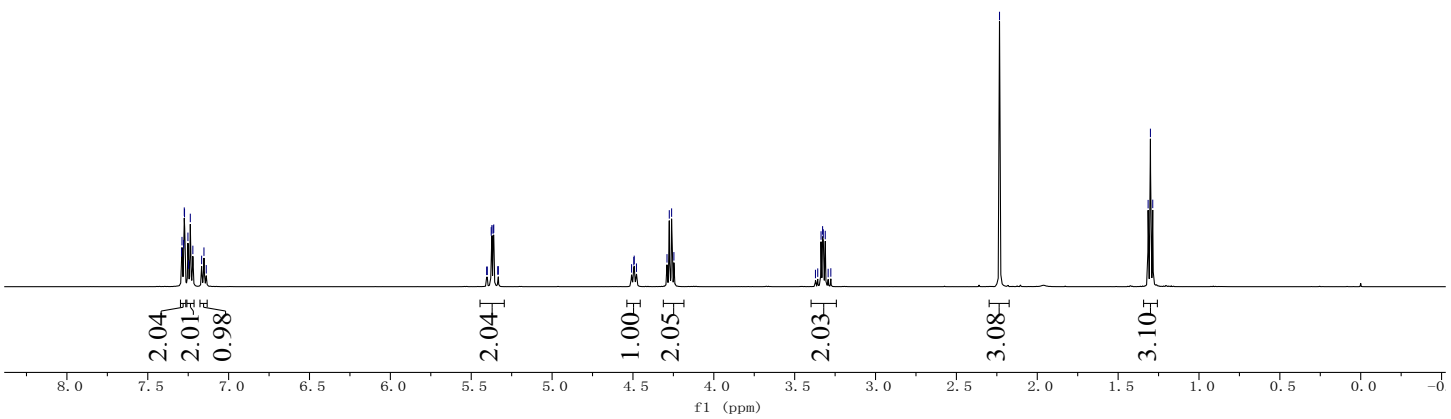

${ }^{1} \mathrm{H}$ NMR spectrum of $\mathbf{1} \mathbf{j a}\left(500 \mathrm{MHz}, \mathrm{CDCl}_{3}\right)$

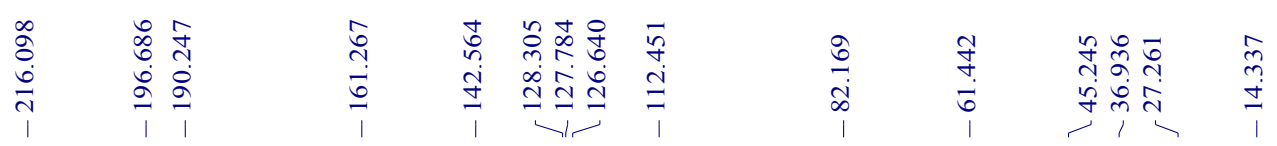<smiles>C=C=C(C(C)=O)C(CC(=O)C(=N)C(=O)OCC)c1ccccc1</smiles>
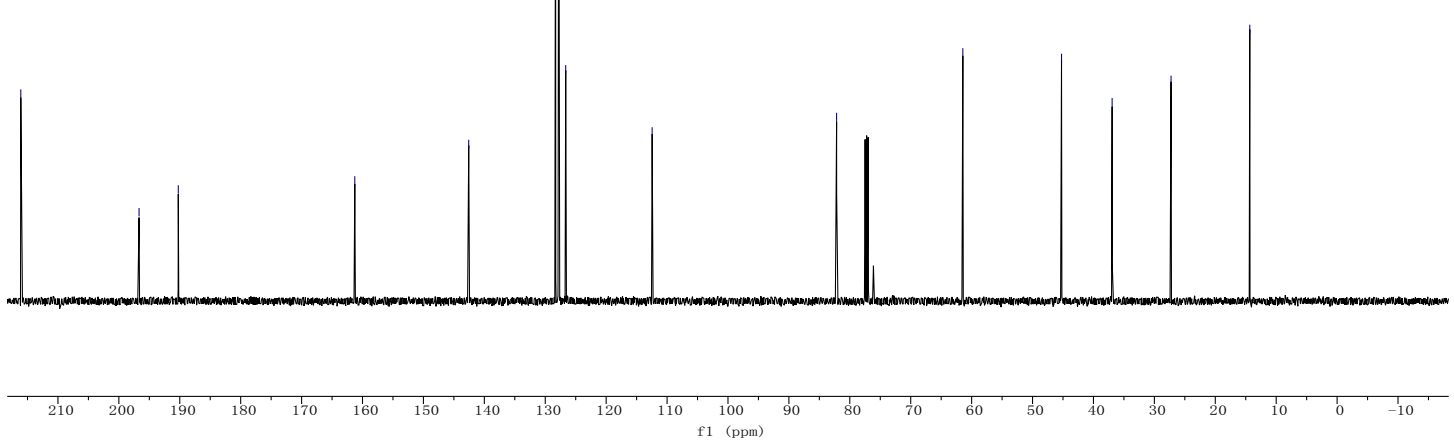

${ }^{13} \mathrm{C}\{1 \mathrm{H}\}$ NMR spectrum of $\mathbf{1 j a}\left(125.8 \mathrm{MHz}, \mathrm{CDCl}_{3}\right)$ 
<smiles>CC(=O)C(=Cc1ccccc1)C(CC(=O)C(=[N+])c1ccccc1)c1ccccc1</smiles>

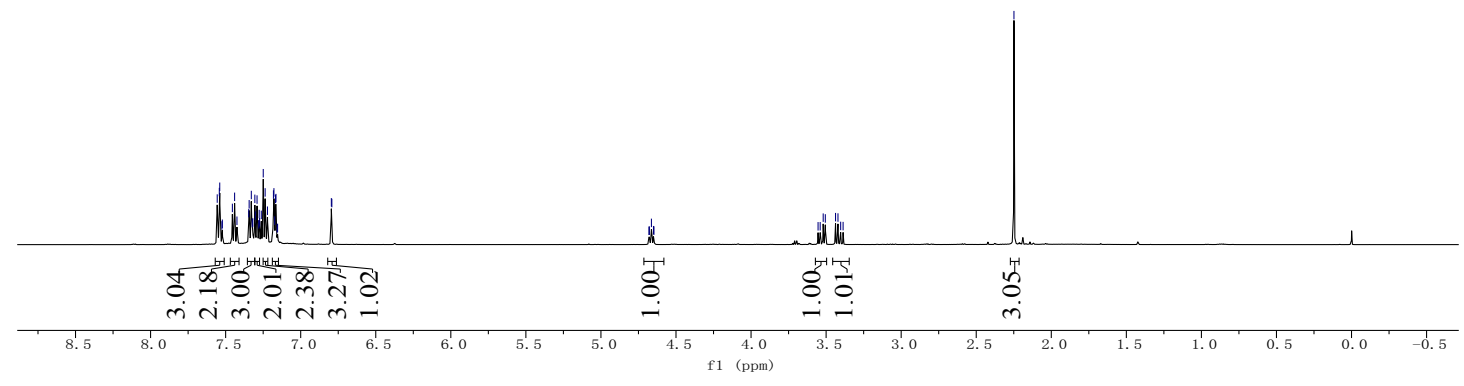

${ }^{1} \mathrm{H}$ NMR spectrum of $\mathbf{1 a b}\left(500 \mathrm{MHz}, \mathrm{CDCl}_{3}\right)$

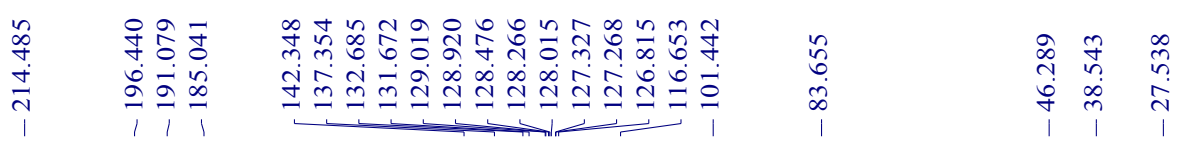<smiles>CC(=O)C(=Cc1ccccc1)C(CC(=O)C(=N)C(=O)c1ccccc1)c1ccccc1</smiles>
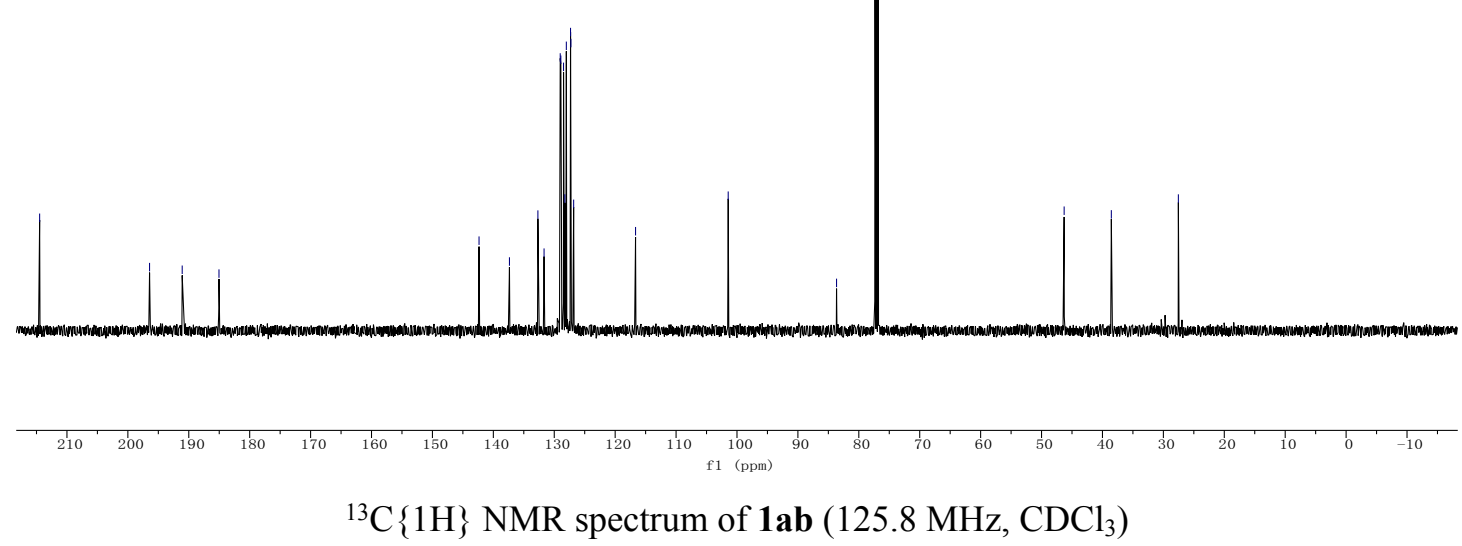
(c)

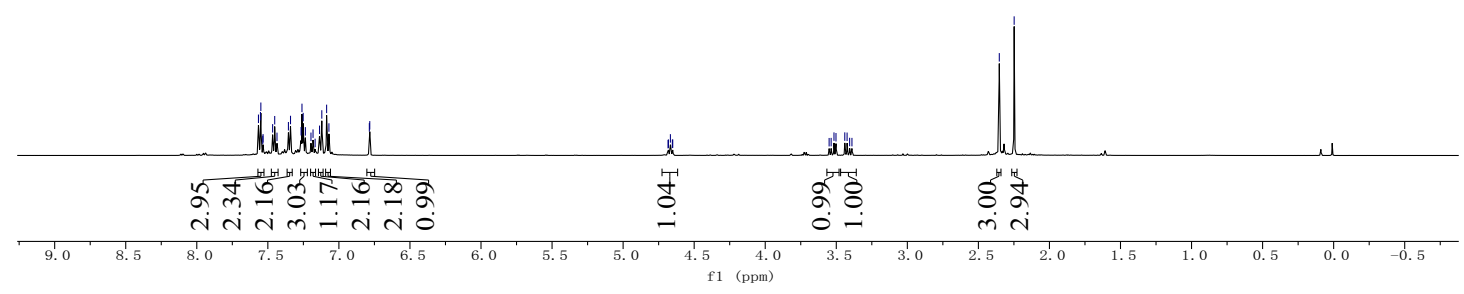

${ }^{1} \mathrm{H}$ NMR spectrum of $\mathbf{1 b b}\left(500 \mathrm{MHz}, \mathrm{CDCl}_{3}\right)$

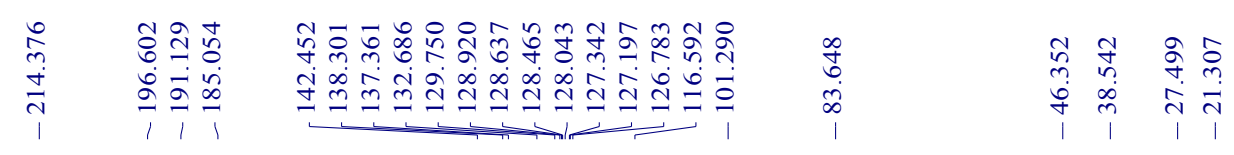<smiles>CC(=O)C(=Cc1ccc(C)cc1)C(CC(=O)C(=N)c1ccccc1)c1ccccc1</smiles>
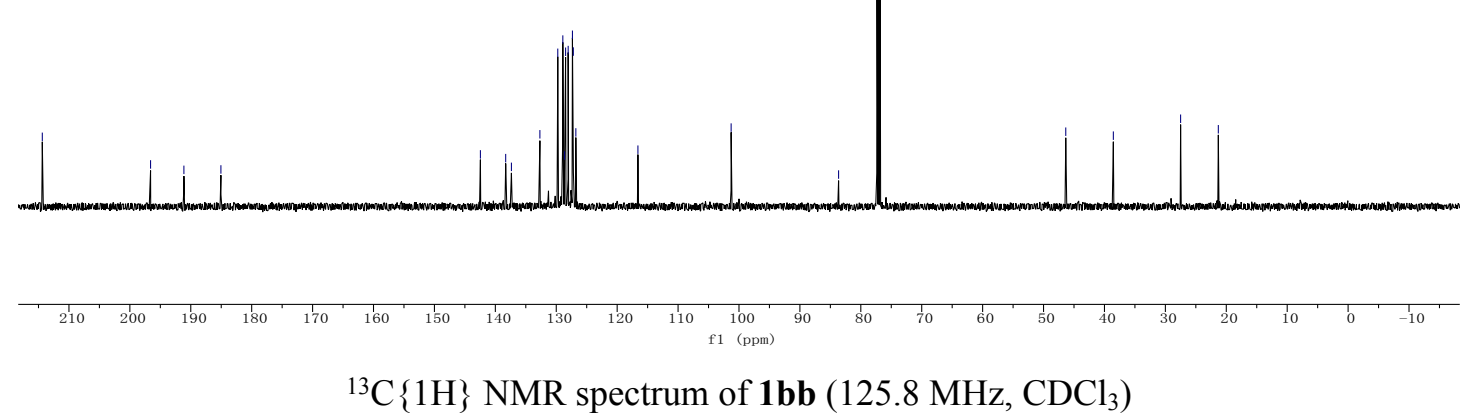

${ }^{13} \mathrm{C}\{1 \mathrm{H}\}$ NMR spectrum of $\mathbf{1 b b}\left(125.8 \mathrm{MHz}, \mathrm{CDCl}_{3}\right)$ 
<smiles>CC(=O)C(=Cc1ccc(F)cc1)C(CC(=O)C(=N)c1ccccc1)c1ccccc1</smiles>

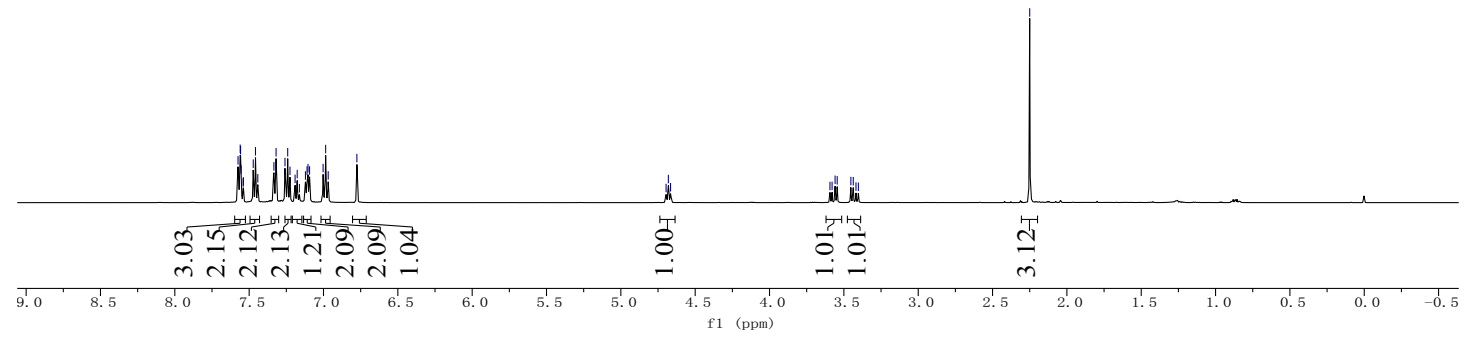

${ }^{1} \mathrm{H}$ NMR spectrum of $\mathbf{1 c b}\left(500 \mathrm{MHz}, \mathrm{CDCl}_{3}\right)$
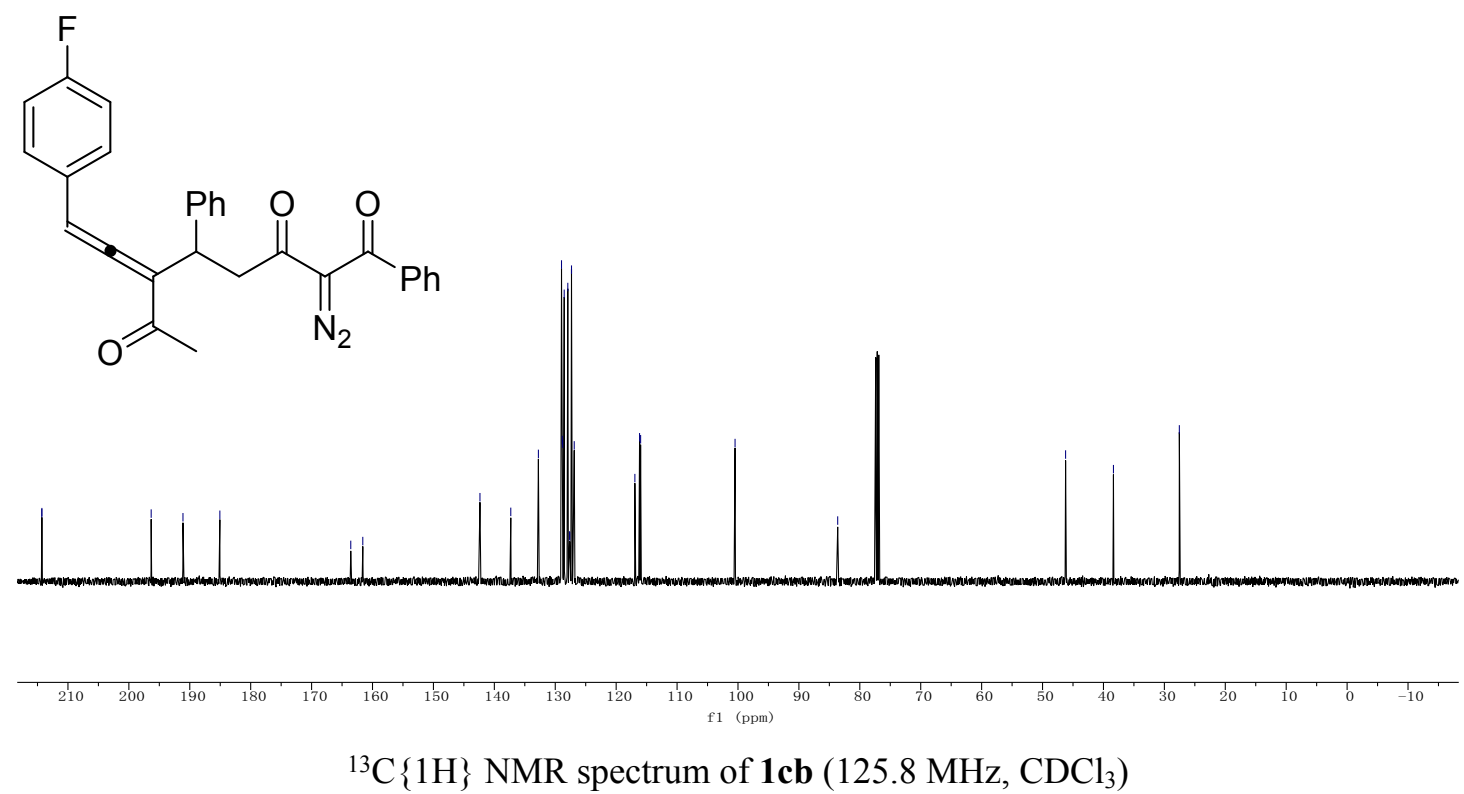

${ }^{13} \mathrm{C}\{1 \mathrm{H}\}$ NMR spectrum of $\mathbf{1 c b}\left(125.8 \mathrm{MHz}, \mathrm{CDCl}_{3}\right)$ 
<smiles>CC(=O)C(=Cc1ccsc1)C(CC(=O)C(=N)C(=O)c1ccccc1)c1ccccc1</smiles>

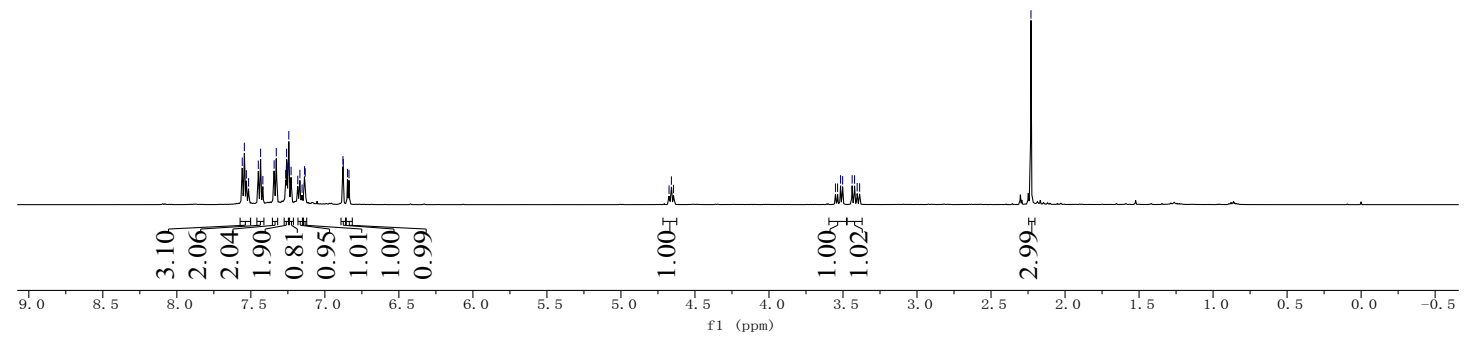

${ }^{1} \mathrm{H}$ NMR spectrum of $\mathbf{1 d b}\left(500 \mathrm{MHz}, \mathrm{CDCl}_{3}\right)$
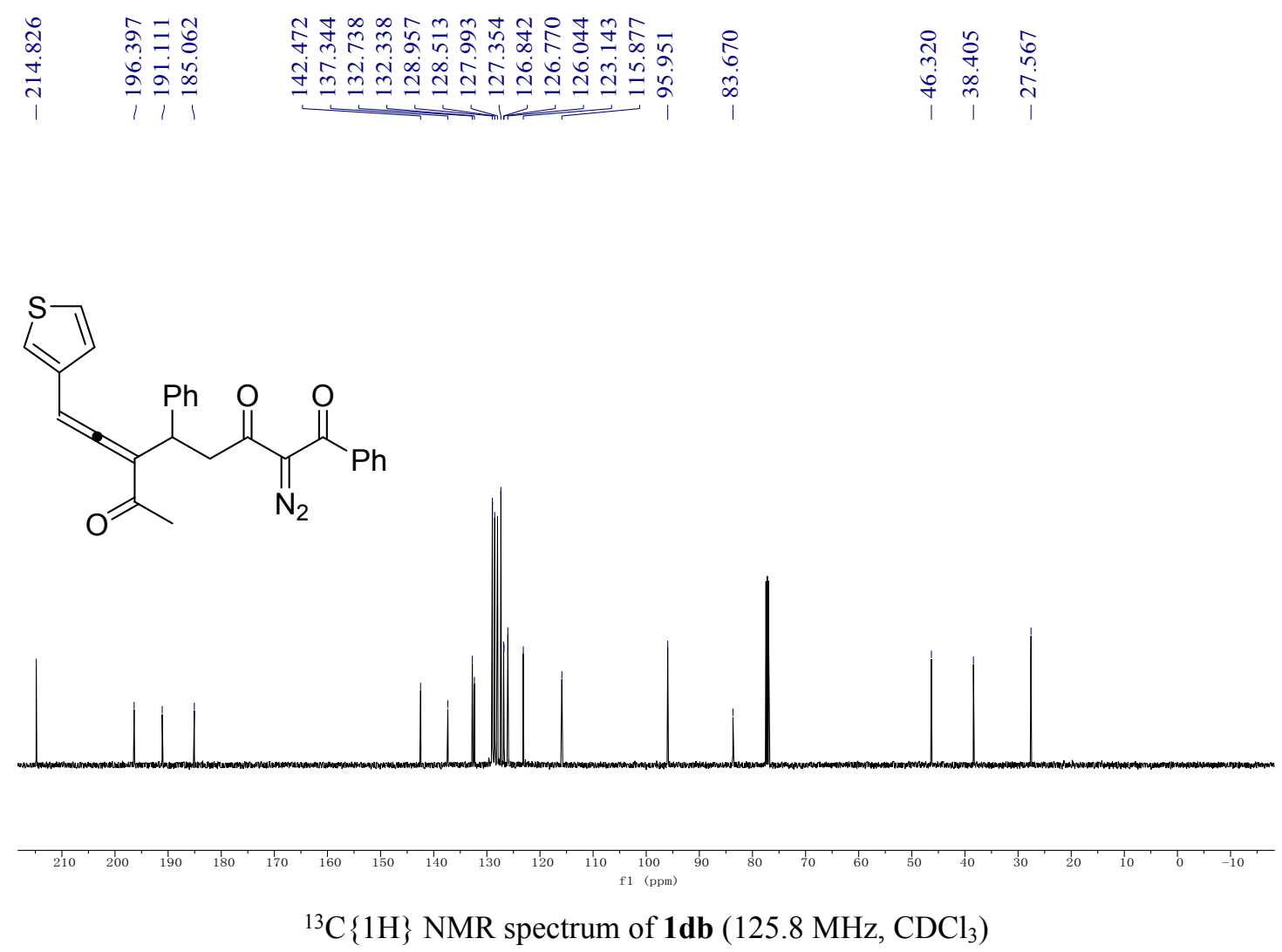

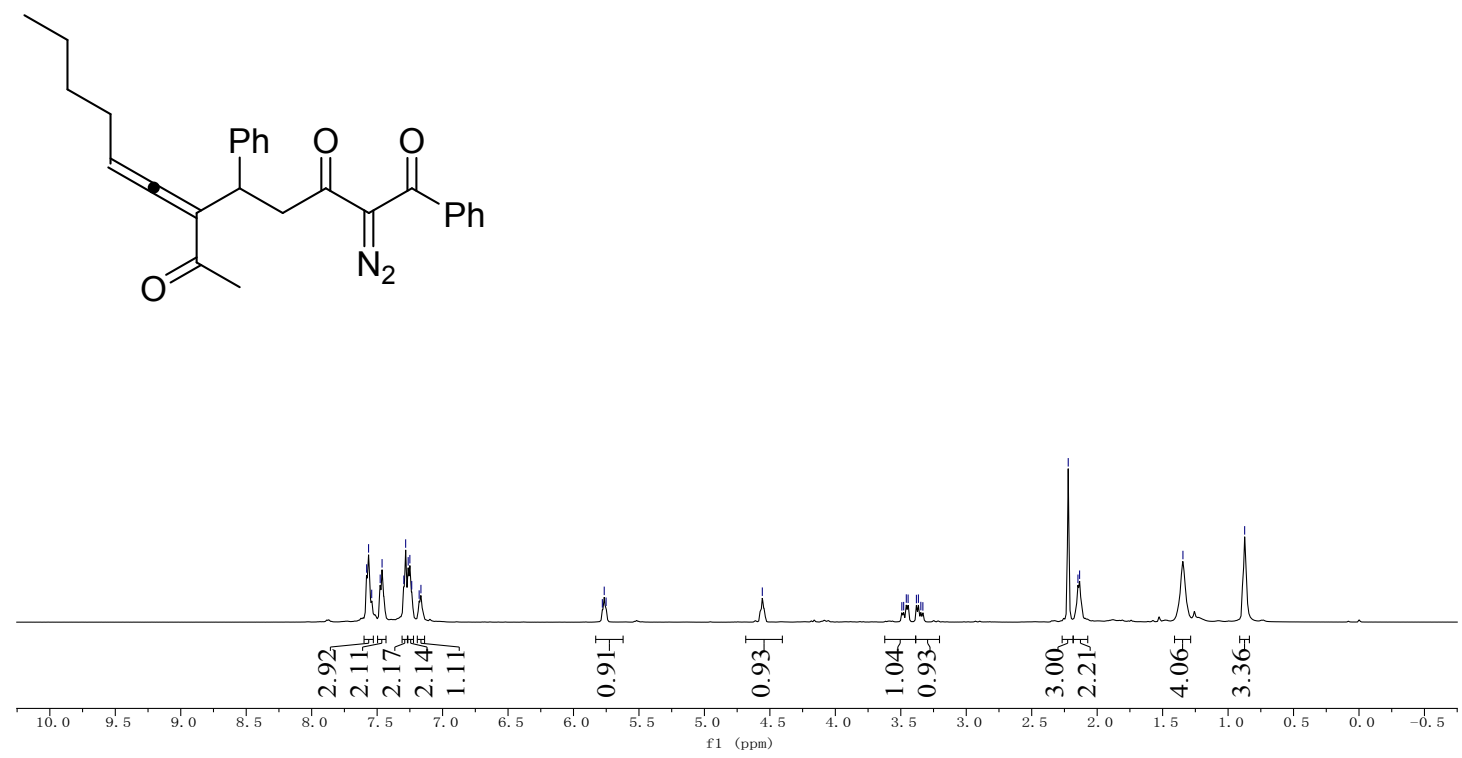

${ }^{1} \mathrm{H}$ NMR spectrum of $\mathbf{1 e b}\left(500 \mathrm{MHz}, \mathrm{CDCl}_{3}\right)$

\begin{tabular}{|c|c|c|c|c|}
\hline 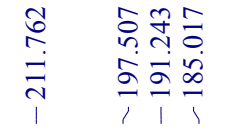 & 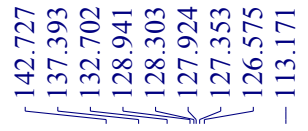 & $\begin{array}{c}\mathcal{O} \\
+ \\
\infty \\
\infty \\
i\end{array}$ & 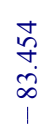 & 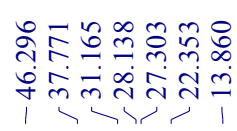 \\
\hline
\end{tabular}<smiles>CCCCC=C=C(C(C)=O)C(CC(=O)C(=N)c1ccccc1)c1ccccc1</smiles>
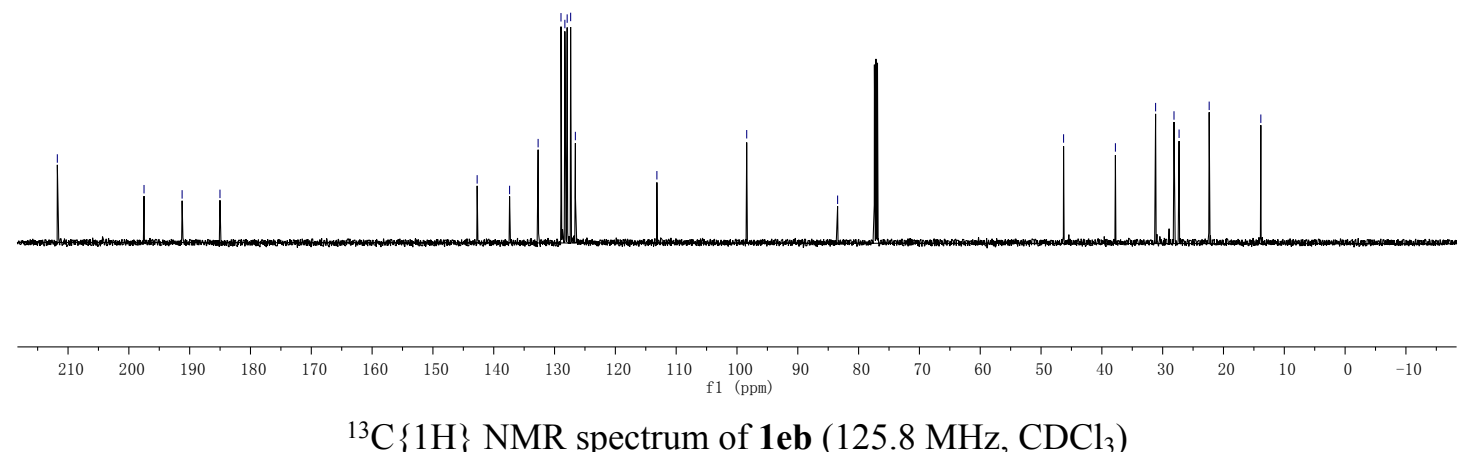

${ }^{13} \mathrm{C}\{1 \mathrm{H}\} \mathrm{NMR}$ spectrum of $\mathbf{1 e b}\left(125.8 \mathrm{MHz}, \mathrm{CDCl}_{3}\right)$ 
<smiles>CC(=O)C(=CC(C)(C)C)C(CC(=O)C(=N)c1ccccc1)c1ccccc1</smiles>

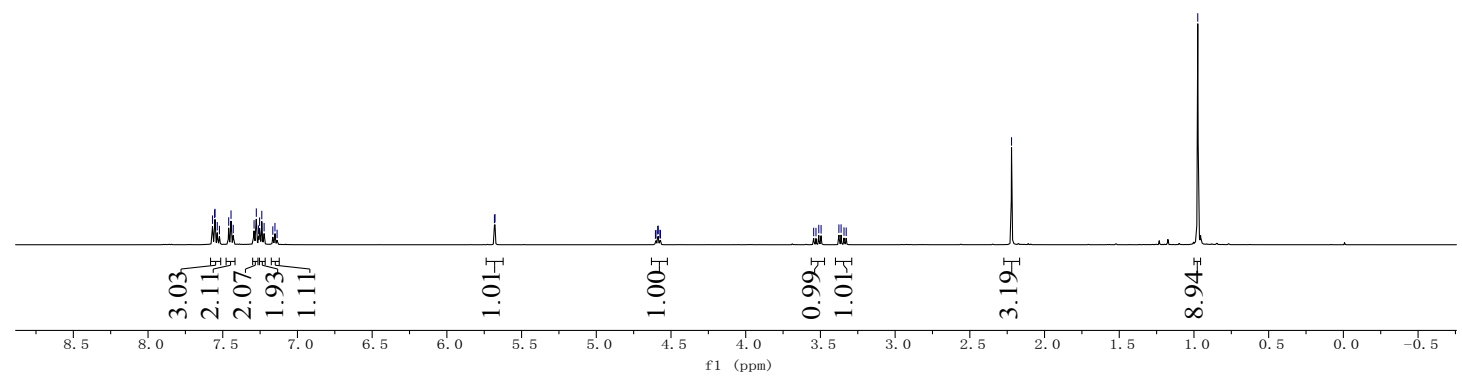

${ }^{1} \mathrm{H}$ NMR spectrum of $\mathbf{1 f b}\left(500 \mathrm{MHz}, \mathrm{CDCl}_{3}\right)$

\begin{tabular}{|c|c|c|}
\hline 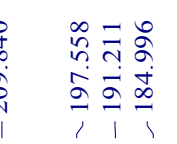 & 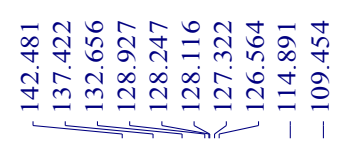 & 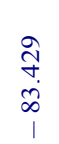 \\
\hline
\end{tabular}
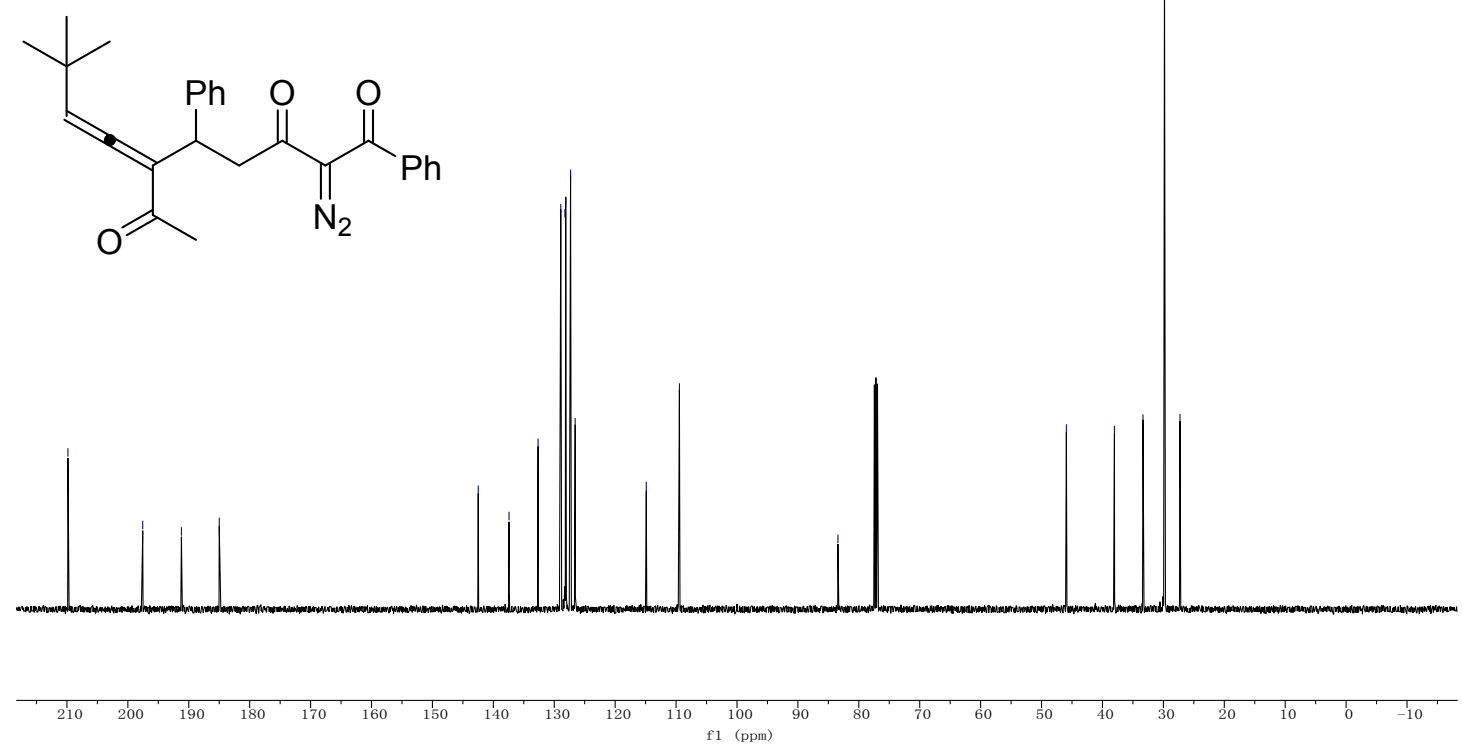

${ }^{13} \mathrm{C}\{1 \mathrm{H}\}$ NMR spectrum of $\mathbf{1 f b}\left(125.8 \mathrm{MHz}, \mathrm{CDCl}_{3}\right)$ 
<smiles>C=C=C(C(C)=O)C(CC(=O)C(=N)C(=O)c1ccccc1)c1ccccc1</smiles>

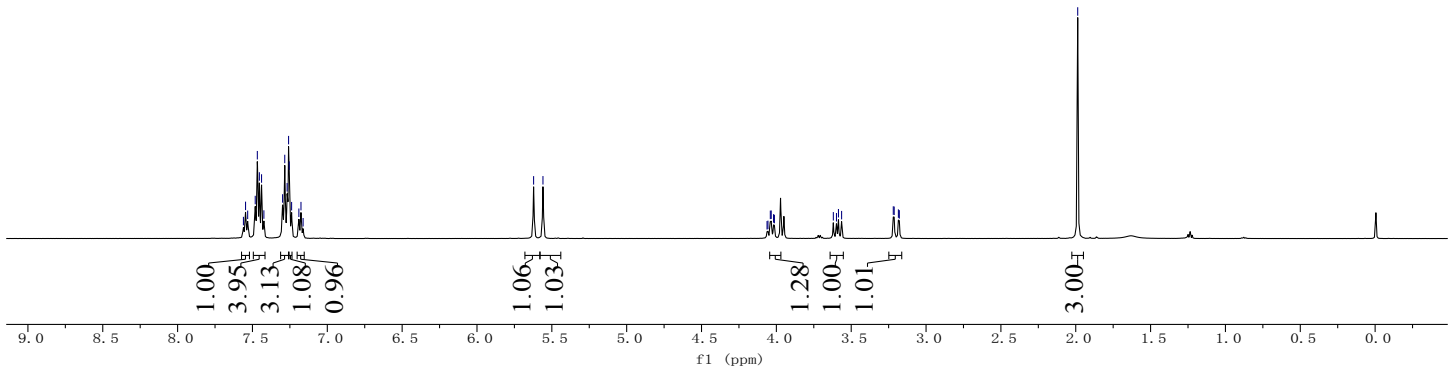

${ }^{1} \mathrm{H}$ NMR spectrum of $\mathbf{1 g b}\left(500 \mathrm{MHz}, \mathrm{CDCl}_{3}\right)$

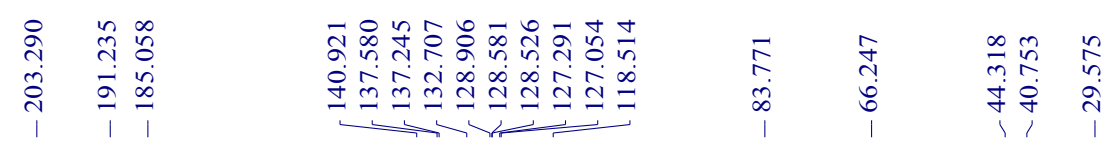<smiles>C=C=C(C(C)=O)C(CC(=O)C(=N)c1ccccc1)c1ccccc1</smiles>
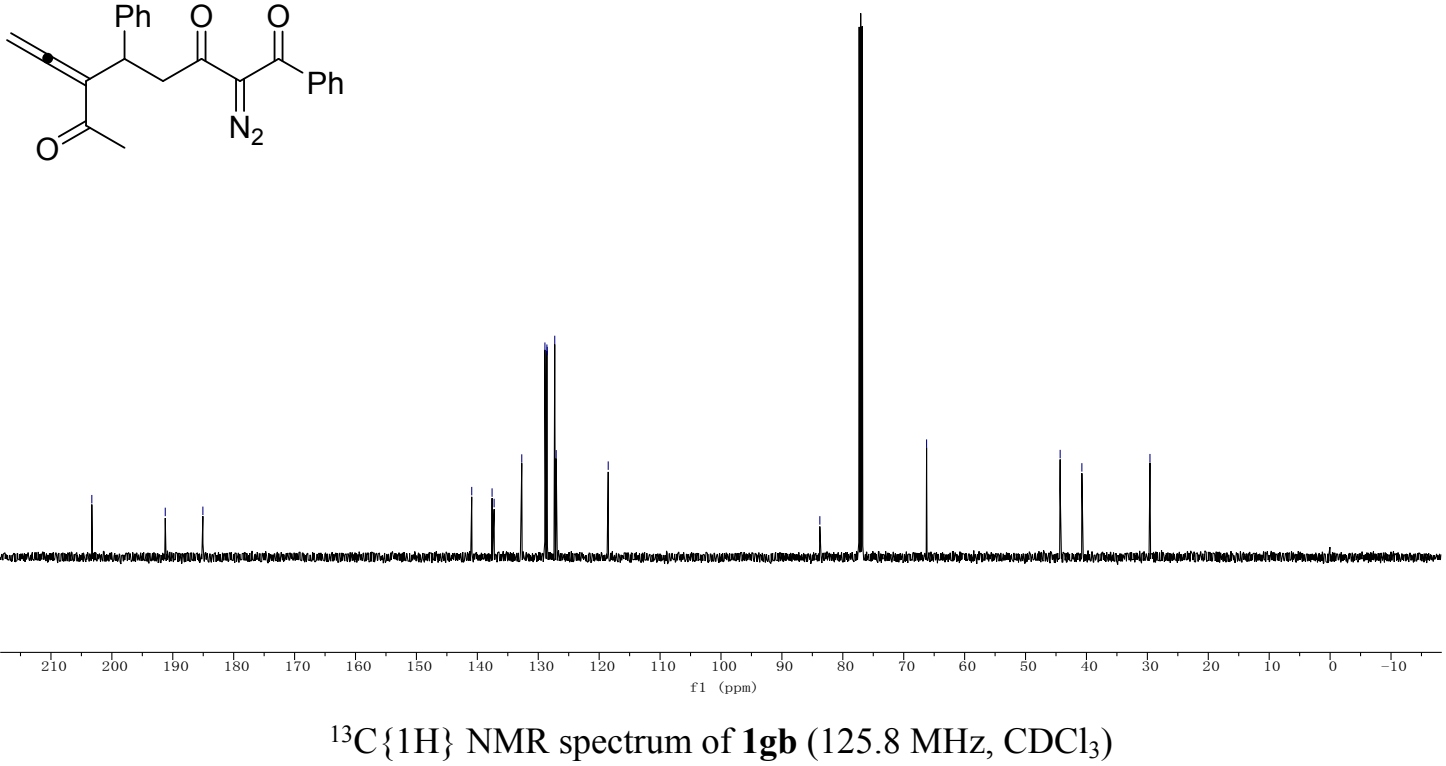


\section{The X-Ray Crystal Structure of $\mathbf{4 e a}$}

Table S1 The single crystal data of 4ea

\begin{tabular}{|c|c|c|}
\hline Bond precision: & $\mathrm{C}-\mathrm{C}=0.0031 \AA$ & Wavelength $=0.71073$ \\
\hline \multirow[t]{2}{*}{ Cell: } & $b=10.647(3)$ & $c=16.385(4)$ \\
\hline & beta $=93.303(13)$ & gamma $=90$ \\
\hline \multicolumn{3}{|c|}{ Temperature: $296 \mathrm{~K}$} \\
\hline & Calculated & Reported \\
\hline Volume & 4113.9(19) & $4113.8(18)$ \\
\hline Space group & $\mathrm{C} 2 / \mathrm{c}$ & $\mathrm{C} 12 / \mathrm{c} 1$ \\
\hline Hall group & $:-C 2 y c$ & $-\mathrm{C} 2 \mathrm{yc}$ \\
\hline Moiety formula & $\mathrm{C}_{24} \mathrm{H}_{21} \mathrm{ClO}_{4}$ & $\mathrm{C}_{24} \mathrm{H}_{21} \mathrm{ClO}_{4}$ \\
\hline Sum formula & $\mathrm{C}_{24} \mathrm{H}_{21} \mathrm{ClO}_{4}$ & $\mathrm{C}_{24} \mathrm{H}_{21} \mathrm{ClO}_{4}$ \\
\hline $\mathrm{Mr}$ & 408.86 & 408.86 \\
\hline Dx,g cm-3 & 1.320 & 1.320 \\
\hline Z & 8 & 8 \\
\hline $\mathrm{Mu}(\mathrm{mm}-1)$ & 0.213 & 0.213 \\
\hline F000 & 1712.0 & 1712.0 \\
\hline F000' & 1713.98 & \\
\hline $\mathrm{h}, \mathrm{k}, \mathrm{lmax}$ & $29,13,20$ & $29,13,20$ \\
\hline Nref & 4052 & 4045 \\
\hline Tmin,Tmax & $0.808,0.808$ & $0.602,0.746$ \\
\hline Tmin' & \multicolumn{2}{|l|}{0.808} \\
\hline \multicolumn{3}{|c|}{ Correction method $=\#$ Reported T Limits: Tmin $=0.602$ Tmax $=0.746$ AbsCorr $=$} \\
\hline \multicolumn{3}{|l|}{ MULTI-SCAN } \\
\hline \multicolumn{2}{|c|}{ Data completeness $=0.998$} & 26.021 \\
\hline \multicolumn{2}{|c|}{$\mathrm{R}($ reflections $)=0.0483(3224)$} & ections $)=0.1400(4045)$ \\
\hline $\mathrm{S}=1.035$ & Npar $=294$ & \\
\hline
\end{tabular}

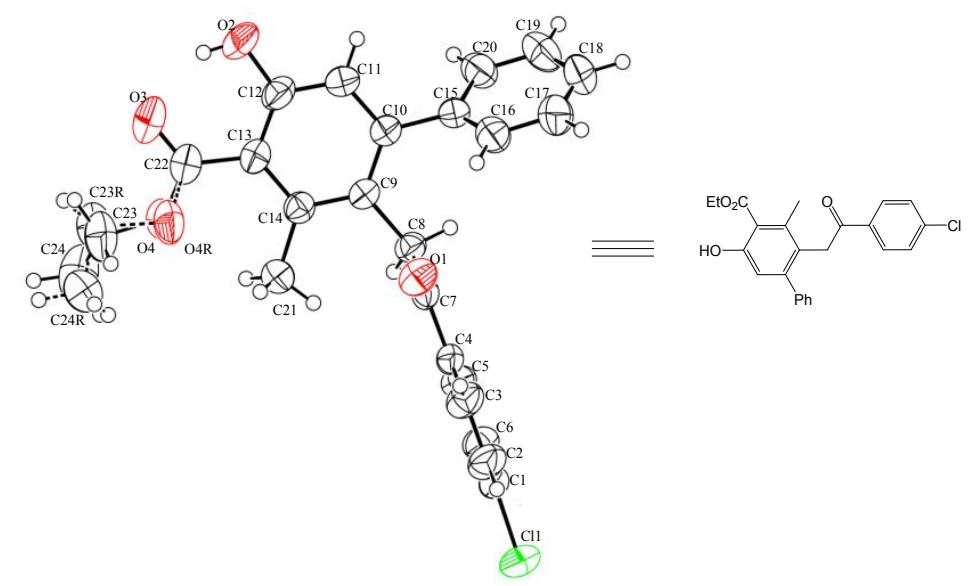

Figure S1 X-ray crystal structure of product 4ea (CCDC 1876455)

Note: the sample was prepared from Dichloromethane/n-hexane $=1: 4$ system via slow evaporation; crystal structure of $\mathbf{4 e a}$ is at $50 \%$ probability levels for the thermal ellipsoid plot. 